\title{
A Prodrug Approach Towards Cancer-Related Carbonic Anhydrase Inhibition
}

Cindy J. Carroux ${ }^{\mathrm{a}}$, Gregory M. Rankin ${ }^{\mathrm{a}}$, Janina Moeker ${ }^{\mathrm{a}}$, Laurent F. Bornaghi ${ }^{\mathrm{a}}$, Kasiram

Katneni $^{\mathrm{b}}$, Julia Morizzi ${ }^{\mathrm{b}}$, Susan A. Charman ${ }^{\mathrm{b}}$, Daniela Vullo ${ }^{\mathrm{c}}$, Claudiu T. Supuran ${ }^{\mathrm{c}, *}$ and Sally-Ann Poulsen ${ }^{\mathrm{a}, *}$

${ }^{a}$ Eskitis Institute for Drug Discovery, Griffith University, Nathan, Queensland 4111, Australia

${ }^{\mathrm{b}}$ Centre for Drug Candidate Optimisation, Monash Institute of Pharmaceutical Sciences, Monash University, Parkville, Victoria 3052, Australia

'Polo Scientifico, Laboratorio di Chimica Bioinorganica, Rm. 188, Università degli Studi di Firenze, Via della Lastruccia 3, 50019 Sesto Fiorentino, Florence, Italy

Keywords carbohydrate, prodrug, esterase, click chemistry, primary sulfonamide, carbonic

anhydrase 


\begin{abstract}
The selective inhibition of cancer-associated human carbonic anhydrase (CA) enzymes, specifically CA IX and XII, has been validated as a mechanistically novel approach towards personalized cancer management. Herein we report the design and synthesis of a panel of 24 novel glycoconjugate primary sulfonamides that bind to the extracellular catalytic domain of CA IX and XII. These compounds were synthesized from variably acylated glycopyranosyl azides and either 3- or 4ethynyl benzene sulfonamide using $\mathrm{Cu}(\mathrm{I})$-catalyzed azide alkyne cycloaddition (CuAAC). The CA enzyme inhibition profile for all compounds was determined, while in vitro metabolic stability, plasma stability and plasma protein binding for a representative set of compounds was measured. Our findings demonstrate the influence of the differing acyl groups on these key biopharmaceutical properties confirming that acyl group protected carbohydrate-based sulfonamides have potential as prodrugs for selectively targeting the extracellular cancer-associated CA enzymes.
\end{abstract}




\section{Introduction}

There are 12 catalytically active isoforms of CA that have been characterized in humans, four of these (CA IV, IX, XII and XIV) are transmembrane anchored proteins with an extracellular active site domain. The role of CA IX and XII was recently shown to be pivotal for $\mathrm{pH}$ regulation to support a microenvironment suited for hypoxic tumor cell survival and proliferation through the conversion of hypoxic cell generated carbon dioxide to bicarbonate and a proton. The effect is to trap protons extracellularly and acidify the extracellular hypoxic microenvironment (supporting tumor invasiveness). $\mathrm{HCO}_{3}{ }^{-}$is transported back into the cell to maintain intracellular $\mathrm{pH}$ (supporting tumor cell survival). ${ }^{1}$ The expression of CA IX and XII is upregulated in many hypoxic tumors, with CA IX usually absent in the corresponding normal tissue and CA XII constitutively expressed in a small selection of normal tissues. ${ }^{2}$ Molecular tools that can selectively bind to or inhibit the cancerassociated CA isozymes in vivo are required to further explore the strong association between CA IX/XII expression and tumor cell survival in the altered microenvironment caused by hypoxia. CA IX-specific monoclonal antibodies are already in Phase III clinical development as both therapeutic and diagnostic agents. ${ }^{3}$ Small molecules can provide improved pharmacokinetic profiles and lower cost over antibodies ${ }^{4}$ and consequently we expect that small molecules which target CA IX and XII would be desirable for targeting hypoxic tumors in vivo. The membrane permeability properties of small molecules targeting CA IX and XII can in principle be employed to take advantage of the cell membrane as a barrier between off-target cytosolic CAs and transmembrane CA IX and XII. CA inhibitors with limited membrane permeability have been developed, ${ }^{5}$ however these compounds are generally hydrophilic and so are likely to lack oral bioavailability. Esters are a common prodrug

moiety employed by the pharmaceutical industry ${ }^{6}$ and ester prodrugs of monosaccharides have been developed to mask polar hydroxyl groups with hydrophobic acyl groups as an approach to enhance oral bioavailability and enable therapeutic concentrations of the prodrug to reach the bloodstream. ${ }^{7}$ 
When in the blood, the ester moiety is susceptible to hydrolysis by carboxylesterases to give the corresponding alcohol and carboxylate, potentially minimizing passive diffusion into cells within tissues. $^{8}$

$\mathrm{Cu}(\mathrm{I})$-catalyzed azide alkyne cycloaddition (CuAAC) to synthesize triazoles has contributed to a renaissance in the chemistry of azides as building block towards higher complexity glycoconjugates. $^{9-11}$ We have recently described the synthesis of glycopyranosyl azides wherein a monosaccharide moiety was peracylated to give $O$-peracylated $\beta$-D-glucopyranosyl (1-5) and $\beta$-Dgalactopyranosyl azides (6-10) comprising acetyl (1 and 6) ${ }^{12}$, propionyl (2 and 7), butanoyl (3 and 8), pentanoyl (4 and 9) and 3-methylbutanoyl (5 and 10) moieties. ${ }^{13}$ We have also established that $1,2,3-$ triazoles are compatible with commonly employed carbohydrate protecting group approaches ${ }^{14}$ and applied CuAAC between the $O$-peracylated glycopyranosyl azides and phenylacetylene to synthesize glycoconjugates as tools to study the effect of different acyl groups on biopharmaceutical properties. We then demonstrated that these carbohydrate building blocks were susceptible to plasma hydrolytic enzymes with serial hydrolysis of the carbohydrate ester moieties to form a fully deprotected and hydrophilic glycoconjugate. Other work in our research group has applied CuAAC to synthesize CA inhibitors. ${ }^{5 c, 5 d, 5 f, 5 g, 15}$ In the present study we combine the merits of these earlier investigations by employing the variably acylated glycopyranosyl azide building blocks 1-10 for the first time in a medicinal chemistry campaign to synthesize glycoconjugates that target CA. We assess the potential of these compounds as prodrugs for a hydrophilic glycoconjugate benzene sulfonamide compound as an approach to selectively target the active site of extracellular CA IX and XII over cytosolic CAs.

\section{Results and Discussion}


Compound Design and Synthesis. The 24 glycoconjugate compounds (13-36) of this study comprise three core structural elements: (i) a sugar moiety (glucose or galactose); (ii) a 1,2,3-triazole moiety; and (iii) a benzene sulfonamide group (meta- or para- substituted). The sugar moiety provides a common prodrug template, while the benzene sulfonamide group is a component of classical small molecule CA inhibitors wherein the sulfonamide anion is able to coordinate to the CA active site $\mathrm{Zn}^{2+}$ to block the endogenous reaction. ${ }^{1,16}$ The triazole linker serves as a biocompatible, non-labile covalent spacer between the sugar and benzene sulfonamide groups. ${ }^{15 a, 17}$ The 24 library members are in four sub-libraries that each comprises six compounds with an identical sugar and benzene sulfonamide group but differing in the sugar protecting group. The sub-libraries consist of a fully deprotected compound $(\mathbf{1 3}, \mathbf{1 9}, 25$ or 31) and the corresponding acylated compounds, including acetyl (14, 20, 26 or 32), propionyl (15, 21, 27 or 33), butanoyl (16, 22, 28 or 34), pentanoyl (17, 23, 29 or 35) or 3-methylbutanoyl $(\mathbf{1 8}, \mathbf{2 4}, 30$ or $\mathbf{3 6})$ acyl groups. The acylated compounds $(\mathbf{1 4 - 1 8 , 2 0 -}$ 24, 26-30, 32-36) were synthesized by the reaction of glycopyranosyl azides 1-10 with either 4ethynyl benzene sulfonamide (11) or 3-ethynyl benzene sulfonamide (12) using CuAAC, Scheme 1. Lengthy reaction times were required for the CuAAC reaction with the more lipophilic glycopyranosyl azides owing to lower solubility in the reaction solvent. The synthesis of compounds 1-11 has been reported previously by ourselves, ${ }^{5 h, 13}$ while compound $\mathbf{1 2}$ has been reported by others. ${ }^{18}$ Compounds 13, 19, 25 and 31 are the fully deacylated glycoconjugates that result from the complete hydrolysis of acyl groups of the corresponding parent compounds 14-18, 20-24, 26-30 and 32-36, respectively. These compounds were however synthesized from the acetylated analogues $\mathbf{1 4}$, 20, 26 and 32 using Zemplén ${ }^{19}$ conditions, Scheme 1. Esterase hydrolysis of compounds 14-18, 2024, 26-30 and 32-36 to yield the fully deprotected compound (13, 19, 25 or 31, respectively) may take place sequentially, forming multiple tri-acylated, di-acylated and mono-acylated glycoconjugates as intermediates, this is shown for the glucoconjugates, Scheme 2. 
Scheme 1. Synthesis of variably acylated glycoconjugate primary sulfonamide compounds 13-36.

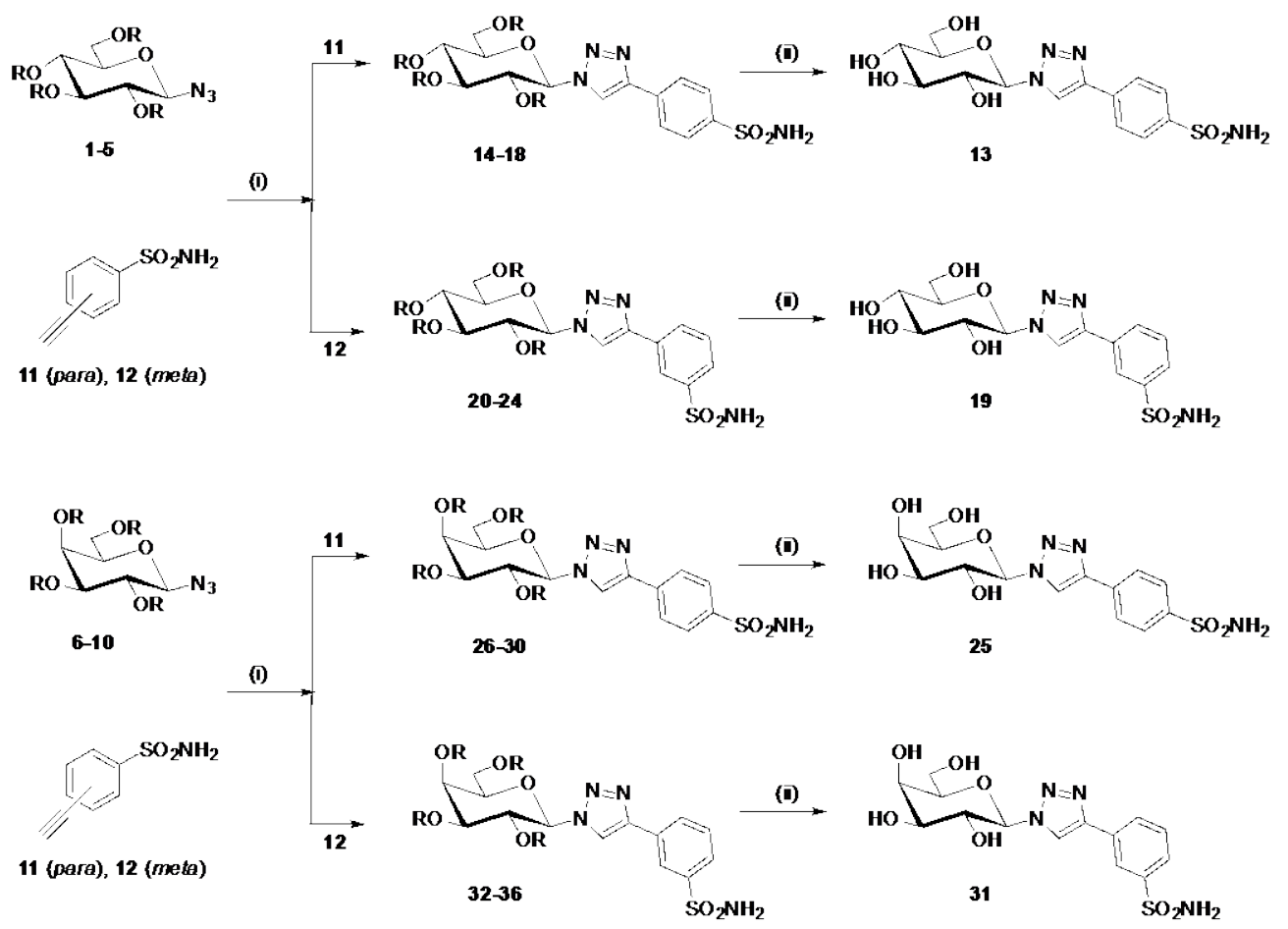

Reagents and Conditions: (i) CuAAC: $\beta$-D-glycopyranosyl azide 1-10 (1 equiv.), alkyne 11 or 12 (1.1 equiv.), $\mathrm{CuSO}_{4} .5 \mathrm{H}_{2} \mathrm{O}$ (0.2 equiv.), sodium ascorbate (0.4 equiv.), $\mathrm{H}_{2} \mathrm{O}: \mathrm{EtOH}$ (1:1 to 1:5), $60{ }^{\circ} \mathrm{C}$. (ii) 14, 20, 26 or 32, NaOMe in $\mathrm{MeOH}(25 \% \mathrm{w} / \mathrm{v}), \mathrm{MeOH}$, rt. 
Scheme 2. Sequential hydrolysis of ester groups of glucoconjugates 14-18 and 20-24 to form the fully deacylated compounds 13 and 19, respectively.

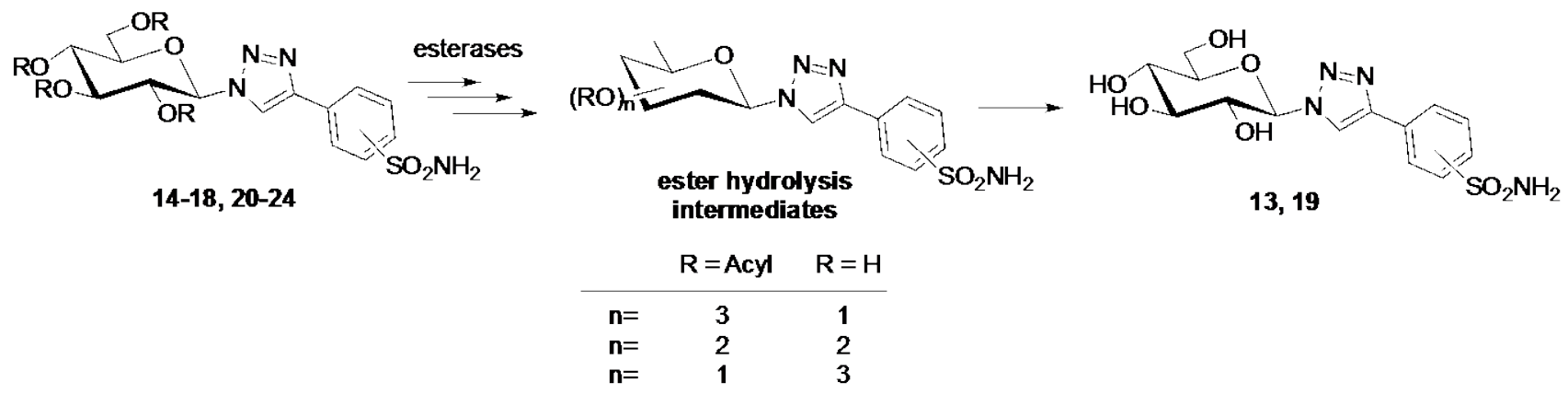

CA Inhibition. The enzyme inhibition data for ethynyl benzene sulfonamides 11 and 12, and glycoconjugate sulfonamides 13-36 were measured for the off-target cytosolic hCA I and II, for the off-target membrane anchored hCA XIV and for the target tumor-associated hCA IX and XII, by analysis of the hydration activity of carbonic anhydrase, ${ }^{20}$ Table 1 . Similarly to acetazolamide (AZA), the glycoconjugate sulfonamides 13-36 and ethynyl benzene sulfonamides 11 and 12 generally displayed poorest inhibition towards the off-target hCA I. For most glycoconjugates hCA I inhibition constants $\left(K_{\mathrm{i}}\right)$ were in the micromolar range and compounds were typically one order of magnitude weaker inhibitors of this isozyme than of hCA II, IX, XII and XIV. The parent nonglycoconjugate compounds (11 and 12) displayed submicromolar inhibition of hCA II, IX, XII and XIV, while the glycoconjugate sulfonamides (13-36) $K_{\mathrm{i}}$ values spanned low nanomolar to low micromolar at hCA II, IX, XII and XIV. There were no consistent SAR trends for the differing acyl groups so that any of the acylated glycoconjugates could serve as a prodrug for the corresponding fully deprotected glycoconjugates. For the glucose series of sulfonamides (13-24) the deprotected glucose analogues 13 and $\mathbf{1 9}$ displayed selectivity for the target hCA IX over the off-target hCA II, with notably improved inhibition of hCA IX than for all of the corresponding parent acylated 
analogues (15-18, 20-24), and similar inhibition of hCA XII compared to acylated analogues 15-18, 20-24. This SAR supports the potential viability of an acyl ester glucoconjugate prodrug approach to selectively target the inhibition of cancer-related CAs over cytosolic off-target CAs. The initial acylated glucoconjugate prodrug (15-18, 20-24) should provide for good oral absorption and low CA inhibition, while the unmasked prodrug generates a hydrophilic glucoconjugate (13 or 19) that should be selective towards overexpressed hCA IX and XII due to a combination of limited membrane permeability and improved $K_{\mathrm{i}}$. In contrast, the deprotected galactose compounds 25 and 31 did not exhibit this optimal balance of SAR between ester prodrug (26-30, 32-36) and unmasked compound (25 or 31) to achieve a useful profile in the context of a prodrug approach. The unmasked compounds 25 and 31 inhibited off-target hCA II 19- and 9-fold better than hCA IX, respectively, while the acylated compounds inhibited hCA IX and XII an order of magnitude better (27), 2- to 4fold better (28) or similarly $(29,30,32-36)$ to the deprotected compounds 25 and 31 . This differing SAR between the glucose and galactose series of glycoconjugate sulfonamides demonstrates that the structure of the carbohydrate moiety of compounds, although not directly impacting on the sulfonamide anion-zinc cation interaction, can have a profound impact on CA inhibition profile. The stereochemical difference between these epimers must lead to an altered interaction(s) of the ligand with the outer residues of the CA active site. We have previously observed in an unrelated set of glycoconjugate benzene sulfonamides that glucose and galactose tail moieties also gave this powerful discrimination between CA isozyme inhibition and selectivity. ${ }^{5 \mathrm{~d}}$

Table 1. Inhibition data of hCA isozymes I, II, IX and XII with compounds 11-36 and the established drug and CA inhibitor, acetazolamide (AZA). 


\begin{tabular}{|c|c|c|c|c|c|c|}
\hline \multirow{2}{*}{ Compd } & \multirow{2}{*}{ Substituents $^{a}$} & \multicolumn{5}{|c|}{$K_{\mathrm{i}}(\mathrm{nM})^{b}$} \\
\hline & & hCA I & hCA II & hCA IX & hCA XII & hCA XIV \\
\hline AZA & - & 250 & 12 & 25 & 5.7 & 41 \\
\hline 11 & - & 1080 & 71.6 & 54.0 & 44.5 & 63.9 \\
\hline 12 & - & 843 & 66.1 & 65.7 & 29.7 & 118 \\
\hline 13 & Glc, OH, para & 9.4 & 380 & 78.9 & 40.1 & 50.3 \\
\hline 14 & Glc, acetyl, para & 4400 & 9.1 & 120 & 67.1 & 104 \\
\hline 15 & Glc, propionyl, para & 2020 & 71.1 & 475 & 7.4 & 256 \\
\hline 16 & Glc, butanoyl, para & 1795 & 76.3 & 633 & 50.2 & 119 \\
\hline 17 & Glc, pentanoyl, para & 9226 & 573 & 951 & 52.7 & 176 \\
\hline 18 & Glc, methylbutanoyl, para & 9441 & 440 & 426 & 52.0 & 40.1 \\
\hline 19 & Glc, OH, meta & 9450 & 621 & 78.1 & 85.4 & 23.7 \\
\hline 20 & Glc, acetyl, meta & 2245 & 261 & 480 & 55.1 & 45.3 \\
\hline 21 & Glc, propionyl, meta & 2216 & 79.4 & 874 & 73.2 & 78.4 \\
\hline 22 & Glc, butanoyl, meta & 2936 & 181 & 583 & 71.9 & 67.0 \\
\hline 23 & Glc, pentanoyl, meta & 9165 & 174 & 402 & 44.1 & 137 \\
\hline 24 & Glc, methylbutanoyl, meta & 9833 & 661 & 724 & 61.4 & 452 \\
\hline 25 & Gal, OH, para & 7950 & 6.7 & 126 & 36.3 & 20.1 \\
\hline 26 & Gal, acetyl, para & 4300 & 8.7 & 110 & 9.7 & 15.8 \\
\hline 27 & Gal, propionyl, para & 78.8 & 8.1 & 5.1 & 5.4 & 10.9 \\
\hline
\end{tabular}




\begin{tabular}{c|c|c|c|c|c|c}
\hline $\mathbf{2 8}$ & Gal, butanoyl, para & 257 & 6.9 & 51.8 & 9.5 & 35.6 \\
$\mathbf{2 9}$ & Gal, pentanoyl, para & 2130 & 73.6 & 335 & 31.0 & 67.3 \\
$\mathbf{3 0}$ & Gal, methylbutanoyl, para & 3205 & 37.9 & 374 & 23.3 & 15.9 \\
$\mathbf{3 1}$ & Gal, OH, meta & 6452 & 69.1 & 590 & 35.0 & 44.5 \\
$\mathbf{3 3}$ & Gal, acetyl, meta & 3823 & 421 & 943 & 61.9 & 50.1 \\
$\mathbf{3 4}$ & Gal, propionyl, meta & 2351 & 253 & 126 & 53.3 & 58.9 \\
$\mathbf{3 5}$ & Gal, butanoyl, meta & 83.1 & 6.3 & 69.2 & 61.1 & 76.0 \\
$\mathbf{3 6}$ & Gal, pentanoyl, meta & 73.8 & 8.0 & 800 & 43.2 & 148 \\
\hline
\end{tabular}

${ }^{a}$ Glc $=$ glucose, Gal $=$ galactose

${ }^{b}$ Errors in the range of $\pm 5 \%$ of the reported value, from three determinations.

In vitro ADME. As the glucose series of compounds proved of interest with respect to SAR a representative sub-library of these compounds, 13-18, was used for in vitro biopharmaceutical property determination. We first determined the in vitro stability of the sub-library 13-18 in human plasma at $37{ }^{\circ} \mathrm{C}$, Table 2. The characteristic molecular weight for the potential hydrolysis intermediates and compound $\mathbf{1 3}$ (as a reference compound for complete hydrolysis) allowed plasma stability to be monitored using mass spectrometry, Figure 1. Compound $\mathbf{1 3}$ remained stable in human plasma over the course of the incubation $(4 \mathrm{~h})$ indicating stability of the underlying glycoconjugate benzene sulfonamide scaffold. The acetyl, propionyl and butanoyl analogues (14, 15 and 16) were rapidly degraded with estimated half lives of 7, 3, and 25 min, respectively, while the pentanoyl (17) and the 3-methylbutanoyl (18) compounds remained stable in human plasma over the course of the incubation. The serial hydrolysis of the ester moieties to variable tri-, di- and mono-acylated ester products, respectively, formed en route to the fully hydrolysed compound $\mathbf{1 3}$, was confirmed by 
mass spectrometry, Figure 1. We recently reported in vitro ADME properties for analogues of 13-18 that have a phenyl substituent in place of the phenyl sulfonamide substituent. ${ }^{13}$ These compounds exhibited the same acyl group plasma stability profile with estimated half lives of 55, 26, and 555 min for the acetyl, propionyl and butanoyl analogues, respectively. The half-lives are however 8- to 25-fold longer than for the corresponding sulfonamides, meaning that the sulfonamides were more labile toward plasma esterases.

Protein binding values of the test compounds 14-18 were estimated by comparison of their chromatographic retention properties on an immobilized human serum albumin (HSA) column to those for a series of standard compounds, Table $2 .^{21}$ As expected the increased protein binding paralleled increased lipophilicity of the acyl groups of the underlying sugar template. In addition the compounds with higher HSA binding (17 and 18, >99\% HSA bound) were also the more stable in human plasma (half-life $>250 \mathrm{~min}$ ). This inverse relationship indicates that the extent of plasma protein binding may influence the plasma stability profile observed for this subset of variable acyl group protected carbohydrate-based sulfonamides. This may be a desirable characteristic for these molecules, minimising the potential for binding to off target red blood cell hCA I and II, while increasing the circulation half-life. The fully deacylated compound $\mathbf{1 3}$ had low HSA binding (13\%).

We then determined the in vitro metabolic stability of compounds 13-18 in human liver microsomes (HLMs) in both the presence and absence of NADPH, the cofactor required for oxidative metabolism by CYP450s, Table 2. No degradation was detected for the fully deacylated (13) or for the more lipophilic pentanoyl (17) and 3-methylbutanoyl (18) compounds; this indicates that 13, 17 and 18 are likely to have low CYP-mediated metabolic clearance in vivo. In comparison the acetyl, propionyl and butanoyl compounds (14, 15 and 16) were degraded in HLMs in both the presence and absence 
of cofactor, indicative of non-CYP450 metabolism. The fully hydrolysed compound $\mathbf{1 3}$ as well as various mono-, di- and tri-acylated intermediates were detected, a finding indicating that HLM degradation is a result of compound susceptibility to hydrolytic enzymes such as microsomal esterases.

The cLog P results imply that compounds 13 and 14 (cLog $\mathrm{P}=-1.75$ and +0.01 , respectively) should exhibit poor passive permeation across gastrointestinal epithelial cells, while compound 15 should exhibit good permeability (cLog $\mathrm{P}=+2.81$ ). Caco-2 cell monolayers were used in an attempt to measure the in vitro permeability of compounds 13-15. Compounds 16-18 were not tested owing to poor solubility in the Caco-2 buffer. For compounds $\mathbf{1 3}$ and $\mathbf{1 4}$ mass balance in the diffusion chamber was good (100\% and $81 \%$, respectively) however no test compound was detected in the receptor chamber. These results are in agreement with expectations of poor membrane permeability and indicate minimal retention of compound within the cell monolayer and minimal non-specific adsorption. For compound $\mathbf{1 5}$ the compound was not detected in the receptor chamber, however mass balance was poor (47\%), precluding an accurate assessment of the permeability of 15, and indicating that it is possible that the compound was retained within the membrane monolayer. This result suggests that for this class of compound clogP values approaching 3 may be too high for a good passive permeability. In addition to this low concentrations of the deacylated compound (13) were detected in the donor chamber suggesting that ester hydrolysis had occurred during the experiment. Overall these results indicate that further optimisation of the prodrug permeability properties will be needed, however the unmasked prodrug permeability is as needed to minimise off target binding to intracellular CAs. 
Table 2. cLog P, in vitro human plasma stability, chromatographic HSA binding (cHSA) and in vitro HLM stability data for test glucoconjugate compounds 13-18. ${ }^{a}$

\section{Plasma}

Stability

Compd Mwt $\operatorname{cLog} \mathrm{P}^{b}$

\section{Degradation}

half-life $(\min )^{c}$

HLM Stability

cHSA $^{d}$

(\%

bound)
In vitro CLint

$(\mu \mathrm{L} / \mathrm{min} / \mathrm{mg} \text { protein })^{c}$

(+)NADPH (-)NADPH

\begin{tabular}{ccccccc}
\hline $\mathbf{1 3}$ & 386.4 & -1.75 & $>250$ & 13 & $<7$ & $<7$ \\
$\mathbf{1 4}$ & 554.5 & +0.01 & 7 & 30 & c.n.d. & c.n.d. \\
$\mathbf{1 5}$ & 610.6 & +2.81 & 3 & 77 & c.n.d. & c.n.d. \\
$\mathbf{1 6}$ & 666.7 & +4.59 & 25 & 94 & c.n.d. & c.n.d. \\
$\mathbf{1 7}$ & 722.9 & +6.37 & $>250$ & $>999^{f}$ & $<7$ & $<7$ \\
$\mathbf{1 8}$ & 722.9 & +5.74 & $>250$ & $>999^{f}$ & $<7$ & $<7$
\end{tabular}

${ }^{a}$ Nonstandard abbreviations: cHSA, chromatographic human serum albumin binding; HLM, human liver microsome; $\mathrm{CL}_{\text {int }}$, in vitro intrinsic clearance. ${ }^{b}$ Calculated using Instant JChem 5.12.0. ${ }^{c}$ Values are the average of duplicate incubations. ${ }^{d}$ Values measured using the chromatographic retention times on an immobilized HSA column. ${ }^{e}$ c.n.d. = could not be determined owing to rapid degradation in the absence of co-factor. ${ }^{f}$ Values are estimates only as the chromatographic peaks were broad and retention times could not be accurately determined.

Figure 1. In vitro stability of compounds 14-16 in human plasma at $37^{\circ} \mathrm{C}$, monitored by UPLC-MS. a) Compound 14: A. Parent compound (m/z 555) at the start of the incubation, (B) mono-deacylation products (m/z 513) after 15 minutes, (C) bis-deacylated products (m/z 471) after 30 minutes, (D) tris- 
deacylated products (m/z 429) after 120 minutes and (E) complete deacylated product, (equivalent to compound 13) (m/z 387) after 240 minutes.
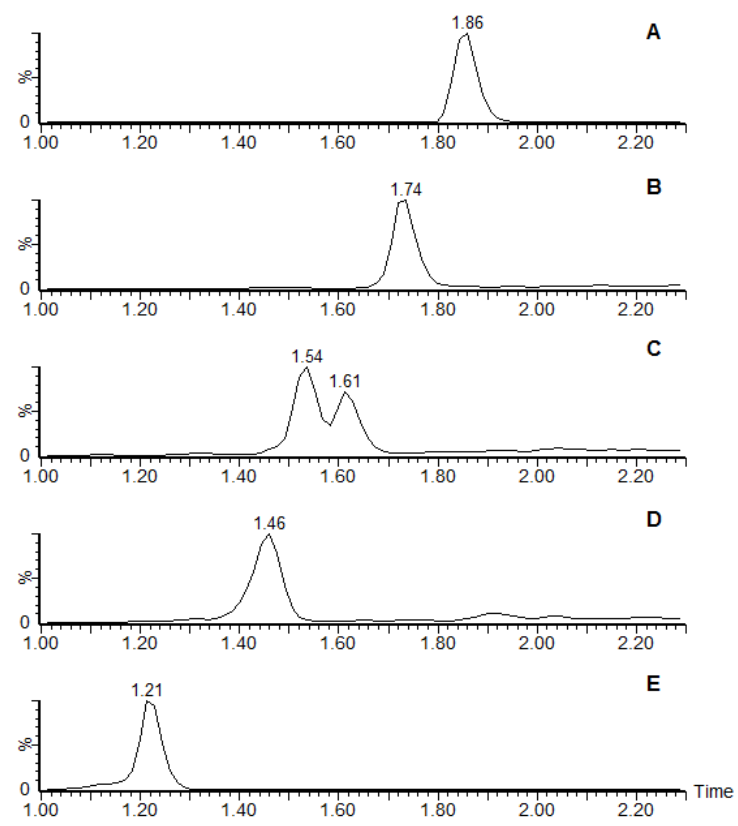

b) Compound 15: A. Parent compound (m/z 611) at the start of the incubation, (B) mono-deacylation products (m/z 555) after 5 minutes, (C) bis-deacylated products (m/z 499) after 30 minutes, (D) trisdeacylated products (m/z 443) after 120 minutes and (E) complete deacylated product, (equivalent to compound 13) (m/z 387) after 240 minutes. 

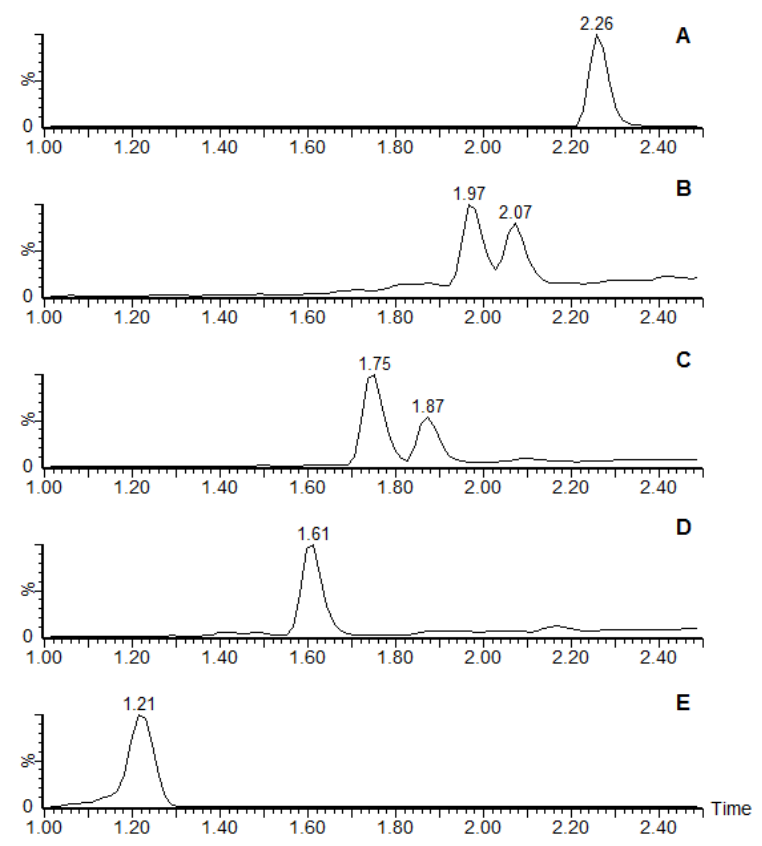
c) Compound 16: A. Parent compound (m/z 667) at the start of the incubation (B) tris-deacylated products (m/z 457) after 120 minutes and (C) complete deacylated product, (equivalent to compound 13) (m/z 387) after 240 minutes. Mono and bis-deacylated products were not detected.
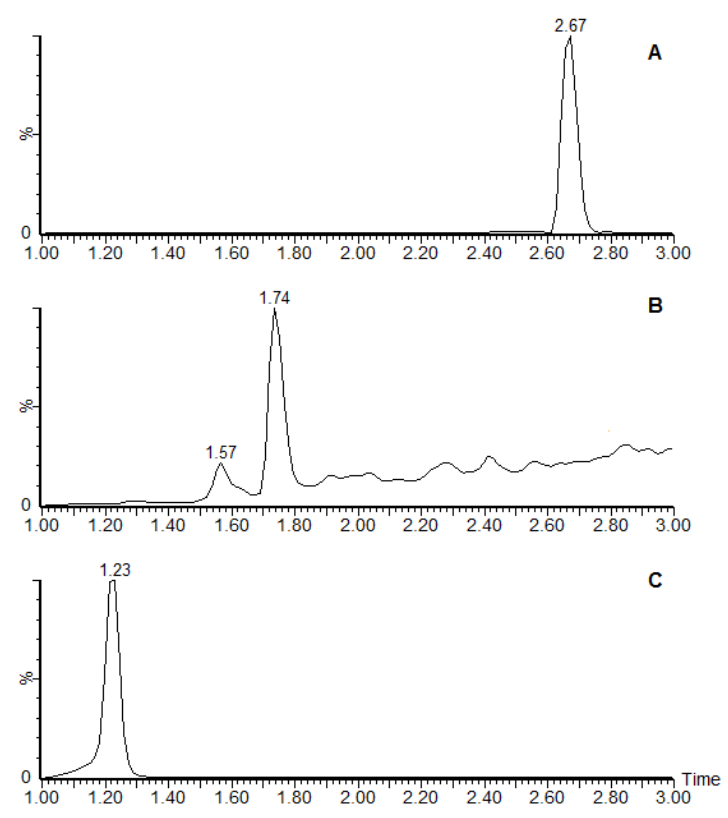

\section{Conclusion}

The ideal profile for an oral prodrug targeting cancer-related hCA IX and XII is good membrane permeability and poor CA inhibition, with the compound unmasked once in circulation. The unmasked compound should then exhibit the opposing characteristics of poor membrane permeability and good CA inhibition. Compounds in the glucose series of sulfonamides (13-18) displayed the needed SAR profile to fulfill these criteria as evidenced by hCA $K_{\mathrm{i}}$ values. The acetyl, propionyl and butanoyl analogues 14-16 were degraded in human plasma and HLMs consistent with esterase processing, to form the fully deacylated compound $\mathbf{1 3}$ that remained stable in these environments. The propionyl analogue $\mathbf{1 5}$ has a cLog $\mathrm{P}$ value of +2.81 consistent with expected good membrane permeability while compound 13 has a cLog $\mathrm{P}$ value of -1.75 , consistent with poor membrane permeability. The measured membrane permeability for $\mathbf{1 3}$ in a Caco-2 cell model 
confirmed negligible membrane permeability, while compound $\mathbf{1 5}$ may be retained in the membrane and future efforts will focus on optimizing this compound. These findings provide a beneficial guide to the impact of these acyl groups on glycoconjugate prodrug stability and performance. In conclusion, the SAR and biopharmaceutical characteristics measured for the panel of 24 glycoconjugate sulfonamides in this study highlights the potential viability of an ester-based prodrug approach to selectively target the inhibition of hCA IX and XII over off-target cytosolic CAs.

\section{Experimental Section}

General Chemistry. All starting materials and reagents, including per-O-acetylated sugars, were purchased from commercial suppliers. All solvents were available commercially dried or dried prior to use. Reaction progress was monitored by TLC using silica gel-60 F254 plates with detection by short wave UV fluorescence $(\lambda=254 \mathrm{~nm})$ and staining with sulfuric acid stain $\left(5 \% \mathrm{H}_{2} \mathrm{SO}_{4}\right.$ in ethanol) and/or orcinol stain (1 $\mathrm{g}$ of orcinol monohydrate in a mixture of EtOH: $\mathrm{H}_{2} \mathrm{O}: \mathrm{H}_{2} \mathrm{SO}_{4}$ 72.5:22.5:5). Silica gel flash chromatography was performed using silica gel $60 \AA$ (230-400 mesh). NMR $\left({ }^{1} \mathrm{H},{ }^{13} \mathrm{C}\left\{{ }^{1} \mathrm{H}\right\}\right.$, gCOSY and HSQC) spectra were recorded on a $500 \mathrm{MHz}$ spectrometer at 30 ${ }^{\circ} \mathrm{C}$. Chemical shifts for ${ }^{1} \mathrm{H}$ and ${ }^{13} \mathrm{C}$ NMR spectra obtained in DMSO- $d_{6}$ are reported in ppm relative to residual solvent proton $(\delta=2.50 \mathrm{ppm})$ and carbon $(\delta=39.5 \mathrm{ppm})$ signals, respectively. Chemical shifts for ${ }^{1} \mathrm{H}$ NMR spectra obtained in $\mathrm{CDCl}_{3}$ are reported in ppm relative to residual solvent proton ( $\delta=7.26 \mathrm{ppm}$ ). Multiplicity is indicated as follows: s (singlet); d (doublet); t (triplet); m (multiplet); dd (doublet of doublet); ddd (doublet of doublet of doublet); b (broad). Coupling constants are reported in hertz (Hz). Melting points are uncorrected. Mass spectra (low and high resolution) were recorded using electrospray as the ionization technique in positive ion and/or negative ion modes as stated. All MS analysis samples were prepared as solutions in methanol. Optical rotations were measured at $25{ }^{\circ} \mathrm{C}$ and reported as an average of ten measurements. Purity of all compounds was $\geq$ 
95\% as determined by HPLC with both UV and ELSD detection. ${ }^{1} \mathrm{H}$ and ${ }^{13} \mathrm{C}$ NMR spectra of compounds 15-24, 27-36 are provided in supporting information. Glycoconjugates are named in accordance with the recommendations of the IUPAC-IUBMB Joint Commission on Biochemical Nomenclature: "Nomenclature of Carbohydrates (Recommendations 1996)" (http://www.chem.qmul.ac.uk/iupac/2carb/). The synthesis of the $\beta$-D-glycopyranosyl azides has been reported by us previously. ${ }^{13}$

General Procedure 1. CuAAC reaction conditions. A mixture of the $\beta$-D-glycopyranosyl azide (1.0 equiv.) and the alkyne (1.1 equiv.) was suspended in a $\mathrm{H}_{2} \mathrm{O} / \mathrm{EtOH}$ mixture (1:1 to 1:5, $10 \mathrm{~mL}$ ). A solution of sodium ascorbate $\left(0.4\right.$ equiv) in water $(0.5 \mathrm{~mL})$, followed by a solution of $\mathrm{CuSO}_{4} .5 \mathrm{H}_{2} \mathrm{O}$ (0.2 equiv) in water $(0.5 \mathrm{~mL})$ was successively added. The bright yellow suspension was stirred vigorously at $60{ }^{\circ} \mathrm{C}$, as needed for full consumption of the azide or no further change as indicated by TLC. The crude product was filtered, washed with $\mathrm{H}_{2} \mathrm{O}$ and redissolved in EtOAc. The organic solution was dried over $\mathrm{MgSO}_{4}$, filtered and concentrated under reduced pressure. Compounds were purified by column chromatography as described below.

General Procedure 2. Deacetylation of carbohydrate moieties. Fully deprotected sugar derivatives were prepared by treating the corresponding acylated precursor in dry $\mathrm{MeOH}$ (final concentration of $\sim 0.1-0.2 \mathrm{M}$ ) with methanolic sodium methoxide (25\% w/v, 100-140 $\mu \mathrm{L} ; \mathrm{pH} 12$ ). The reaction was left to stir until full deacylation was evident by TLC $\left(\mathrm{CH}_{3} \mathrm{CN}: \mathrm{H}_{2} \mathrm{O} 9: 1 ; 30\right.$ min overnight). Neutralization of the solution by Amberlite IR-120- $\mathrm{H}^{+}$ion-exchange resin, followed by filtration (the resin is washed several times with methanol) and evaporation of the filtrate to dryness, generally afforded analytically pure compounds. 


\section{4-[4"'-(Aminosulfonyl)phenyl]-1-(ק-D-glucopyranosyl)-1H-1,2,3-triazole (13)}

The title compound 13 was prepared from compound 14 (185 mg, $0.334 \mathrm{mmol})$ according to general procedure 2 and isolated as a white solid (111 mg, 93\%). $R_{f}=0.61\left(\mathrm{CH}_{3} \mathrm{CN} / \mathrm{H}_{2} \mathrm{O} 9: 1\right) .{ }^{1} \mathrm{H}$ NMR was in agreement with the data reported in the literature. ${ }^{5 \mathrm{~h}}$

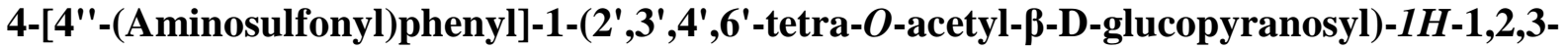

\section{triazole (14)}

The title compound $\mathbf{1 4}$ was prepared from glucopyranosyl azide $\mathbf{1}$ (338 mg, $1.87 \mathrm{mmol}$ ) and alkyne 11 (696 mg, $1.87 \mathrm{mmol}$ ) according to general procedure 1. Purification of the crude product by flash chromatography (EtOAc/Hexane 7:3 to 4:1) afforded 14 (864 mg, 83\% yield) as a white solid. ${ }^{1} \mathrm{H}$ NMR was in agreement with the data reported in the literature. ${ }^{5 h}$

\section{4-[4"'-(Aminosulfonyl)phenyl]-1-(2',3',4',6'-tetra-O-propionyl- $\beta$-D-glucopyranosyl)-1H-1,2,3-}

\section{triazole (15)}

The title compound 15 was prepared from glucopyranosyl azide $\mathbf{2}(154 \mathrm{mg}, 0.36 \mathrm{mmol})$ and alkyne 11 according to general procedure 1 in $3.5 \mathrm{~h}$. Purification of the crude product by flash chromatography (EtOAc/hexane 3:2) afforded $15(168 \mathrm{mg}, 76 \%)$ as an off-white solid. $R_{f}=0.49$ (EtOAc/hexane 3:2). mp 223-225 ${ }^{\circ} \mathrm{C} .[\alpha]^{25}{ }_{\mathrm{D}}-40\left(c=0.061, \mathrm{CHCl}_{3}\right){ }^{1} \mathrm{H}$ NMR (500 MHz, DMSO- $\left.d_{6}\right)$ : $\delta=9.11\left(\mathrm{~s}, 1 \mathrm{H}, \mathrm{CH}_{\text {triazole }}\right), 8.02\left(\mathrm{bd},{ }^{3} J=8.2 \mathrm{~Hz}, 2 \mathrm{H}, \mathrm{CH}_{\text {arom }}\right), 7.92\left(\mathrm{bd},{ }^{3} J=8.2 \mathrm{~Hz}, 2 \mathrm{H}, \mathrm{CH}_{\text {arom }}\right.$ ), $7.38\left(\mathrm{~s}, 2 \mathrm{H}, \mathrm{SO}_{2} \mathrm{NH}_{2}\right), 6.44\left(\mathrm{~d},{ }^{3} J_{1^{\prime}, 2^{\prime}}=8.8 \mathrm{~Hz}, 1 \mathrm{H}, \mathrm{H}_{1^{\prime}}\right), 5.69\left(\mathrm{dd},{ }^{3} J_{2^{\prime}, 3^{\prime}}=9.3 \mathrm{~Hz},{ }^{3} J_{2^{\prime}, 1^{\prime}}=8.8 \mathrm{~Hz}, 1 \mathrm{H}\right.$, $\left.\mathrm{H}_{2^{\prime}}\right), 5.64\left(\mathrm{dd},{ }^{3} J_{3^{\prime}, 4^{\prime}}=9.6 \mathrm{~Hz},{ }^{3} J_{3^{\prime}, 2^{\prime}}=9.3 \mathrm{~Hz}, 1 \mathrm{H}, \mathrm{H}_{3^{\prime}}\right), 5.22\left(\mathrm{dd},{ }^{3} J_{4^{\prime}, 5^{\prime}}=9.7 \mathrm{~Hz},{ }^{3} J_{4^{\prime}, 3^{\prime}}=9.6 \mathrm{~Hz}, 1 \mathrm{H}\right.$, $\left.\mathrm{H}_{\left.4^{\prime}\right)}\right), 4.48-4.44\left(\mathrm{~m}, 1 \mathrm{H}, \mathrm{H}_{5^{\prime}}\right), 4.22\left(\mathrm{dd},{ }^{2} J_{6 a^{\prime}, 6 b^{\prime}}=12.7 \mathrm{~Hz},{ }^{3} J_{6 a^{\prime}, 5^{\prime}}=5.2 \mathrm{~Hz}, 1 \mathrm{H}, \mathrm{H}_{6 \mathrm{a}^{\prime}}\right), 4.12\left(\mathrm{dd},{ }^{2} J_{6 a^{\prime}, 6 b^{\prime}}\right.$ $\left.=12.7 \mathrm{~Hz},{ }^{3} J_{6 b^{\prime}, 5^{\prime}}=2.0 \mathrm{~Hz}, 1 \mathrm{H}, \mathrm{H}_{6 \mathrm{~b}^{\prime}}\right), 2.39-2.20\left(\mathrm{~m}, 6 \mathrm{H}, \mathrm{COCH}_{2} \mathrm{CH}_{3}\right), 2.12-2.01(\mathrm{~m}, 2 \mathrm{H}$, $\mathrm{COCH}_{2} \mathrm{CH}_{3}$ ), $1.03-0.96\left(\mathrm{~m}, 9 \mathrm{H}, \mathrm{COCH}_{2} \mathrm{CH}_{3}\right), 0.78\left(\mathrm{t}, J=7.5 \mathrm{~Hz}, 3 \mathrm{H}, \mathrm{COCH}_{2} \mathrm{CH}_{3}\right.$ ). Assignments 
were confirmed by ${ }^{1} \mathrm{H}-{ }^{1} \mathrm{H}$ gCOSY. ${ }^{13} \mathrm{C}$ NMR (500 MHz, DMSO- $d_{6}$ ): $\delta=173.3,172.8,172.6,171.9$ $\left(4 \times \mathrm{COCH}_{2} \mathrm{CH}_{3}\right), 145.7\left(\mathrm{C}_{1^{\prime \prime} / 4^{\prime \prime}}\right), 143.6\left(\mathrm{C}_{1^{\prime \prime} / 4^{\prime \prime}}\right), 133.1\left(\mathrm{C}_{\text {triazole }}\right), 126.5\left(2 \times \mathrm{CH}_{\text {arom }}\right), 125.5(2 \times$ $\left.\mathrm{CH}_{\text {arom }}\right)$, $121.6\left(\mathrm{CH}_{\text {triazole }}\right), 84.0\left(\mathrm{C}_{1^{\prime}}\right), 73.4\left(\mathrm{C}_{5^{\prime}}\right), 72.0\left(\mathrm{C}_{3^{\prime}}\right), 70.3\left(\mathrm{C}_{2^{\prime}}\right), 67.3\left(\mathrm{C}_{4^{\prime}}\right), 61.5\left(\mathrm{C}_{6^{\prime}}\right)$, 26.7, 26.69, 26.6, $26.4\left(4 \times \mathrm{COCH}_{2} \mathrm{CH}_{3}\right), 8.9,8.84$ (2C), $8.78\left(4 \times \mathrm{COCH}_{2} \mathrm{CH}_{3}\right)$. Assignments were confirmed by ${ }^{1} \mathrm{H}^{13} \mathrm{C}$ gHSQC. LRMS $\left(\mathrm{ESI}^{+}\right): \mathrm{m} / \mathrm{z}=633[\mathrm{M}+\mathrm{Na}]^{+}$. HRMS (ESI) calcd for $\mathrm{C}_{26} \mathrm{H}_{34} \mathrm{~N}_{4} \mathrm{O}_{11} \mathrm{SNa}$ 633.1837, found 633.1853.

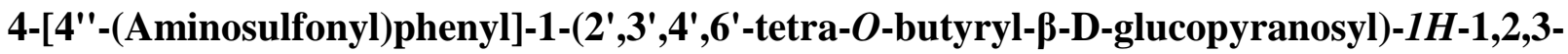

\section{triazole (16)}

The title compound 16 was prepared from glucopyranosyl azide 3 (125 mg, $0.26 \mathrm{mmol}$ ) and alkyne 11 (51 mg, $0.28 \mathrm{mmol}$ ) according to general procedure 1 in 4 days. Purification of the crude product by flash chromatography (EtOAc/hexane 2:3 to 1:1) afforded 16 (85 mg, 49\%) as a pale yellow solid. $R_{f}=0.23$ (EtOAc/hexane 2:3). mp $155-190{ }^{\circ} \mathrm{C}(\mathrm{dec}.) .[\alpha]^{25} \mathrm{D}-42\left(c=0.083, \mathrm{CHCl}_{3}\right) .{ }^{1} \mathrm{H} \mathrm{NMR}$ (500 MHz, DMSO- $d_{6}$ ): $\delta=9.10$ (s, $1 \mathrm{H}, \mathrm{CH}_{\text {triazole }}$ ), 8.00 (bd, ${ }^{3} \mathrm{~J}=8.4 \mathrm{~Hz}, 2 \mathrm{H}, \mathrm{CH}_{\text {arom }}$ ), 7.91 (bd, ${ }^{3} \mathrm{~J}=$ $8.4 \mathrm{~Hz}, 2 \mathrm{H}, \mathrm{CH}_{\text {arom }}$ ), 7.34 (s, 2H, $\left.\mathrm{SO}_{2} \mathrm{NH}_{2}\right), 6.44\left(\mathrm{~d},{ }^{3} J_{1^{\prime}, 2^{\prime}}=8.8 \mathrm{~Hz}, 1 \mathrm{H}, \mathrm{H}_{1^{\prime}}\right), 5.70\left(\mathrm{dd},{ }^{3} J_{2^{\prime}, 3^{\prime}}=9.2 \mathrm{~Hz}\right.$, $\left.{ }^{3} J_{2^{\prime}, 1^{\prime}}=8.8 \mathrm{~Hz}, 1 \mathrm{H}, \mathrm{H}_{2^{\prime}}\right), 5.65\left(\mathrm{dd},{ }^{3} J_{3^{\prime}, 4^{\prime}}=9.6 \mathrm{~Hz},{ }^{3} J_{3^{\prime}, 2^{\prime}}=9.3 \mathrm{~Hz}, 1 \mathrm{H}, \mathrm{H}_{3^{\prime}}\right), 5.23\left(\mathrm{dd},{ }^{3} J_{4^{\prime}, 5^{\prime}}=9.6 \mathrm{~Hz}\right.$, $\left.{ }^{3} J_{4^{\prime}, 3^{\prime}}=9.6 \mathrm{~Hz}, 1 \mathrm{H}, \mathrm{H}_{4^{\prime}}\right), 4.45\left(\mathrm{ddd},{ }^{3} J_{5^{\prime}, 4^{\prime}}=9.6 \mathrm{~Hz},{ }^{3} J_{5^{\prime}, 6 a^{\prime}}=5.0 \mathrm{~Hz},{ }^{3} J_{5^{\prime}, 6 b^{\prime}}=2.1 \mathrm{~Hz}, 1 \mathrm{H}, \mathrm{H}_{5^{\prime}}\right), 4.18(\mathrm{dd}$, $\left.{ }^{2} J_{6 a^{\prime}, 6 b^{\prime}}=12.6 \mathrm{~Hz},{ }^{3} J_{6 a^{\prime}, 5^{\prime}}=5.0 \mathrm{~Hz}, 1 \mathrm{H}, \mathrm{H}_{6 \mathrm{a}^{\mathrm{a}}}\right), 4.13\left(\mathrm{dd},{ }^{2} J_{6 a^{\prime}, 6 b^{\prime}}=12.6 \mathrm{~Hz},{ }^{3} J_{6 b^{\prime}, 5^{\prime}}=2.1 \mathrm{~Hz}, 1 \mathrm{H}, \mathrm{H}_{6 b^{\prime}}\right)$, $2.35-2.23\left(\mathrm{~m}, 4 \mathrm{H}, \mathrm{COCH}_{2} \mathrm{CH}_{2} \mathrm{CH}_{3}\right), 2.19\left(\mathrm{t}, J=7.2 \mathrm{~Hz}, 2 \mathrm{H}, \mathrm{COCH}_{2} \mathrm{CH}_{2} \mathrm{CH}_{3}\right), 2.06-2.01(\mathrm{~m}, 2 \mathrm{H}$, $\mathrm{COCH}_{2} \mathrm{CH}_{2} \mathrm{CH}_{3}$ ), 1.56 - 1.44 (m, 6H, $\mathrm{COCH}_{2} \mathrm{CH}_{2} \mathrm{CH}_{3}$ ), 1.29 - 1.23 (m, 2H, $\mathrm{COCH}_{2} \mathrm{CH}_{2} \mathrm{CH}_{3}$ ), 0.90 - $0.80\left(\mathrm{~m}, 9 \mathrm{H}, \mathrm{COCH}_{2} \mathrm{CH}_{2} \mathrm{CH}_{3}\right.$ ), $0.56\left(\mathrm{t}, \mathrm{J}=7.4 \mathrm{~Hz}, 3 \mathrm{H}, \mathrm{COCH}_{2} \mathrm{CH}_{2} \mathrm{CH}_{3}\right.$ ). Assignments were confirmed by ${ }^{1} \mathrm{H}-{ }^{1} \mathrm{H}$ gCOSY. ${ }^{13} \mathrm{C}$ NMR (500 MHz, DMSO- $\left.d_{6}\right): \delta=172.4,171.8,171.6,170.6(4 \times$ $\left.\mathrm{COCH}_{2} \mathrm{CH}_{2} \mathrm{CH}_{3}\right), 145.7\left(\mathrm{C}_{1^{\prime \prime} / 4^{\prime \prime}}\right), 143.7\left(\mathrm{C}_{1^{\prime \prime} / 4^{\prime \prime}}\right), 133.1\left(\mathrm{C}_{\text {triazole }}\right), 126.5\left(2 \times \mathrm{CH}_{\text {arom }}\right), 125.5(2 \times$ $\left.\mathrm{CH}_{\text {arom }}\right)$, $121.6\left(2 \times \mathrm{CH}_{\text {triazole }}\right), 84.1\left(\mathrm{C}_{1^{\prime}}\right), 73.4\left(\mathrm{C}_{5^{\prime}}\right), 71.8\left(\mathrm{C}_{3^{\prime}}\right), 70.2\left(\mathrm{C}_{2^{\prime}}\right), 67.3\left(\mathrm{C}_{4^{\prime}}\right), 61.4\left(\mathrm{C}_{6^{\prime}}\right), 35.14$, 
35.08, 35.06, $34.8\left(4 \times \mathrm{COCH}_{2} \mathrm{CH}_{2} \mathrm{CH}_{3}\right), 17.71,17.67$ (3C) $\left(4 \times \mathrm{COCH}_{2} \mathrm{CH}_{2} \mathrm{CH}_{3}\right), 13.28,13.26$, 13.2, $12.7\left(\mathrm{COCH}_{2} \mathrm{CH}_{2} \mathrm{CH}_{3}\right)$. Assignments were confirmed by ${ }^{1} \mathrm{H}^{-13} \mathrm{C}$ gHSQC. LRMS $\left(\mathrm{ESI}^{+}\right): \mathrm{m} / \mathrm{z}=$ $689[\mathrm{M}+\mathrm{Na}]^{+}$. HRMS (ESI) calcd for $\mathrm{C}_{30} \mathrm{H}_{42} \mathrm{~N}_{4} \mathrm{O}_{11} \mathrm{SNa}$ 689.2463, found 689.2482.

\section{4-[4' '-(Aminosulfonyl)phenyl]-1-(2',3',4',6'-tetra-O-pentyryl-p-D-glucopyranosyl)-1H-1,2,3-}

\section{triazole (17)}

The title compound 17 was prepared from glucopyranosyl azide 4 (155 mg, $0.290 \mathrm{mmol}$ ) and alkyne 11 (91 mg, $0.50 \mathrm{mmol}$ ) according to general procedure 1 in 6 days. Purification of the crude product by flash chromatography (EtOAc/hexane 3:7 to 2:3) afforded 17 (118 mg, 57\%) as a white solid. $R_{f}=$ 0.08 (EtOAc/hexane 3:7). mp 215-217 ${ }^{\circ} \mathrm{C} .[\alpha]^{25}{ }_{\mathrm{D}}-29\left(c=0.056, \mathrm{CHCl}_{3}\right) .{ }^{1} \mathrm{H}$ NMR $(500 \mathrm{MHz}$, DMSO- $d_{6}$ ): $\delta=9.09$ (s, 1H, $\mathrm{CH}_{\text {triazole }}$ ), 8.01 (bd, ${ }^{3} J=8.4 \mathrm{~Hz}, 2 \mathrm{H}, \mathrm{CH}_{\text {arom }}$ ), 7.92 (bd, ${ }^{3} J=8.4 \mathrm{~Hz}, 2 \mathrm{H}$, $\mathrm{CH}_{\text {arom }}$ ), 7.39 (s, $\left.2 \mathrm{H}, \mathrm{SO}_{2} \mathrm{NH}_{2}\right), 6.44\left(\mathrm{~d},{ }^{3} J_{1^{\prime}, 2^{\prime}}=8.9 \mathrm{~Hz}, 1 \mathrm{H}, \mathrm{H}_{1^{\prime}}\right), 5.70\left(\mathrm{dd},{ }^{3} J_{2^{\prime}, 3^{\prime}}=9.2 \mathrm{~Hz},{ }^{3} J_{2^{\prime}, 1^{\prime}}=8.9\right.$ $\left.\mathrm{Hz}, 1 \mathrm{H}, \mathrm{H}_{2^{\prime}}\right), 5.65\left(\mathrm{dd},{ }^{3} J_{3^{\prime}, 4^{\prime}}=9.4 \mathrm{~Hz},{ }^{3} J_{3^{\prime}, 2^{\prime}}=9.2 \mathrm{~Hz}, 1 \mathrm{H}, \mathrm{H}_{3^{\prime}}\right), 5.23\left(\mathrm{dd},{ }^{3} J_{4^{\prime}, 5^{\prime}}=9.6 \mathrm{~Hz},{ }^{3} J_{4^{\prime}, 3^{\prime}}=9.4\right.$ $\left.\mathrm{Hz}, 1 \mathrm{H}, \mathrm{H}_{4^{\prime}}\right), 4.44\left(\mathrm{ddd},{ }^{3} J_{5^{\prime}, 4^{\prime}}=9.6 \mathrm{~Hz},{ }^{3} J_{5^{\prime}, 6 a^{\prime}}=5.1 \mathrm{~Hz},{ }^{3} J_{5^{\prime}, 6 b^{\prime}}=1.9 \mathrm{~Hz}, 1 \mathrm{H}, \mathrm{H}_{5^{\prime}}\right), 4.17\left(\mathrm{dd},{ }^{2} J_{6 a^{\prime}, 6 b^{\prime}}=\right.$ $\left.12.7 \mathrm{~Hz},{ }^{3} J_{6 a^{\prime}, 5^{\prime}}=5.1 \mathrm{~Hz}, 1 \mathrm{H}, \mathrm{H}_{6 \mathrm{a}^{\prime}}\right), 4.12\left(\mathrm{dd},{ }^{2} J_{6 a^{\prime}, 6 b^{\prime}}=12.7 \mathrm{~Hz},{ }^{3} J_{6 b^{\prime}, 5^{\prime}}=1.9 \mathrm{~Hz}, 1 \mathrm{H}, \mathrm{H}_{6 b^{\prime}}\right), 2.39-2.22$ (m, 4H, $\mathrm{COCH}_{2} \mathrm{CH}_{2} \mathrm{CH}_{2} \mathrm{CH}_{3}$ ), 2.21 (t, $\left.J=7.3 \mathrm{~Hz}, 2 \mathrm{H}, \mathrm{COCH}_{2} \mathrm{CH}_{2} \mathrm{CH}_{2} \mathrm{CH}_{3}\right), 2.10-1.99$ (m, 2H, $\left.\mathrm{COCH}_{2} \mathrm{CH}_{2} \mathrm{CH}_{2} \mathrm{CH}_{3}\right), 1.53-1.39\left(\mathrm{~m}, 6 \mathrm{H}, \mathrm{COCH}_{2} \mathrm{CH}_{2} \mathrm{CH}_{2} \mathrm{CH}_{3}\right), 1.33-1.14$ (m, 8H, $\left.\mathrm{COCH}_{2} \mathrm{CH}_{2} \mathrm{CH}_{2} \mathrm{CH}_{3}\right), \quad 0.98-0.88\left(\mathrm{~m}, 2 \mathrm{H}, \mathrm{COCH}_{2} \mathrm{CH}_{2} \mathrm{CH}_{2} \mathrm{CH}_{3}\right) 0.88-0.80$ (m, 9H, $\mathrm{COCH}_{2} \mathrm{CH}_{2} \mathrm{CH}_{2} \mathrm{CH}_{3}$ ), 0.63 (t, $J=7.3 \mathrm{~Hz}, 3 \mathrm{H}, \mathrm{COCH}_{2} \mathrm{CH}_{2} \mathrm{CH}_{2} \mathrm{CH}_{3}$ ). Assignments were confirmed by ${ }^{1} \mathrm{H}-{ }^{1} \mathrm{H}$ gCOSY. ${ }^{13} \mathrm{C}$ NMR (500 MHz, DMSO- $\left.d_{6}\right): \delta=172.5,171.9,171.8,171.1(4 \times$ $\left.\mathrm{COCH}_{2} \mathrm{CH}_{2} \mathrm{CH}_{2} \mathrm{CH}_{3}\right), 145.7\left(\mathrm{C}_{1^{\prime \prime} / 4^{\prime \prime}}\right), 143.6\left(\mathrm{C}_{1^{\prime \prime} / 4^{\prime \prime}}\right), 133.1\left(\mathrm{C}_{\text {triazole }}\right), 126.4\left(2 \times \mathrm{CH}_{\text {arom }}\right), 125.4(2 \times$ $\left.\mathrm{CH}_{\text {arom }}\right)$, 121.6 $\left(\mathrm{CH}_{\text {triazole }}\right), 84.0\left(\mathrm{C}_{1^{\prime}}\right), 73.4\left(\mathrm{C}_{5^{\prime}}\right), 71.9\left(\mathrm{C}_{3^{\prime}}\right), 70.2\left(\mathrm{C}_{2^{\prime}}\right), 67.4\left(\mathrm{C}_{4^{\prime}}\right), 61.4\left(\mathrm{C}_{6^{\prime}}\right)$, 33.0, 32.9 (2C), $32.7\left(4 \times \mathrm{COCH}_{2} \mathrm{CH}_{2} \mathrm{CH}_{2} \mathrm{CH}_{3}\right), 26.32,26.27$ (3C) $\left(4 \times \mathrm{COCH}_{2} \mathrm{CH}_{2} \mathrm{CH}_{2} \mathrm{CH}_{3}\right), 21.47,21.45$ (2C), $21.1\left(4 \times \mathrm{COCH}_{2} \mathrm{CH}_{2} \mathrm{CH}_{2} \mathrm{CH}_{3}\right), 13.48,13.46,13.41,13.3\left(4 \times \mathrm{COCH}_{2} \mathrm{CH}_{2} \mathrm{CH}_{2} \mathrm{CH}_{3}\right)$. 
Assignments were confirmed by ${ }^{1} \mathrm{H}^{13}{ }^{13} \mathrm{C}$ gHSQC. LRMS $\left(\mathrm{ESI}^{+}\right): \mathrm{m} / \mathrm{z}=723[\mathrm{M}+\mathrm{H}]^{+}$. HRMS (ESI) calcd for $\mathrm{C}_{34} \mathrm{H}_{50} \mathrm{~N}_{4} \mathrm{O}_{11} \mathrm{SNa} 745.3089$, found 745.3061.

\section{4-[4' '-(Aminosulfonyl)phenyl]-1-(2',3',4',6'-tetra-O-(3-methylbutyryl)- $\beta$-D-glucopyranosyl)-1H-}

\section{1,2,3-triazole (18)}

The title compound 18 was prepared from glucopyranosyl azide 5 (260mg, 0.48 mmol) and alkyne 11 (96 mg, 0.53) according to general procedure 1 in 3 days. Purification of the crude product by flash chromatography (EtOAc/hexane 3:7 to 1:1) afforded 18 (65 mg, 23\%) as a white solid. $R_{f}=$ $0.08\left(\right.$ EtOAc/hexane 3:7). $\mathrm{mp}>240{ }^{\circ} \mathrm{C} .[\alpha]^{25}{ }_{\mathrm{D}}^{-49}\left(c=0.072, \mathrm{CHCl}_{3}\right) .{ }^{1} \mathrm{H}$ NMR (500 MHz, DMSO$\left.d_{6}\right): \delta=9.08$ (s, $\left.1 \mathrm{H}, \mathrm{CH}_{\text {triazole }}\right), 8.00$ (bd, $\left.{ }^{3} \mathrm{~J}=8.4 \mathrm{~Hz}, 2 \mathrm{H}, \mathrm{CH}_{\text {arom }}\right), 7.91$ (bd, ${ }^{3} \mathrm{~J}=8.4 \mathrm{~Hz}, 2 \mathrm{H}, \mathrm{CH}_{\text {arom }}$ ), $7.39\left(\mathrm{~s}, 2 \mathrm{H}, \mathrm{SO}_{2} \mathrm{NH}_{2}\right), 6.44\left(\mathrm{~d},{ }^{3} J_{1^{\prime}, 2^{\prime}}=8.8 \mathrm{~Hz}, 1 \mathrm{H}, \mathrm{H}_{1^{\prime}}\right), 5.71\left(\mathrm{dd},{ }^{3} J_{2^{\prime}, 3^{\prime}}=9.2 \mathrm{~Hz},{ }^{3} J_{2^{\prime}, 1^{\prime}}=8.8 \mathrm{~Hz}, 1 \mathrm{H}\right.$, $\left.\mathrm{H}_{2^{\prime}}\right), 5.66\left(\mathrm{dd},{ }^{3} J_{3^{\prime}, 4^{\prime}}=9.3 \mathrm{~Hz},{ }^{3} J_{3^{\prime}, 2^{\prime}}=9.2 \mathrm{~Hz}, 1 \mathrm{H}, \mathrm{H}_{3^{\prime}}\right), 5.23\left(\mathrm{dd},{ }^{3} J_{4^{\prime}, 5^{\prime}},=9.6 \mathrm{~Hz},{ }^{3} J_{4^{\prime}, 3^{\prime}}=9.3 \mathrm{~Hz}, 1 \mathrm{H}\right.$, $\left.\mathrm{H}_{4^{\prime}}\right), 4.44\left(\mathrm{ddd},{ }^{3} J_{5^{\prime}, 4^{\prime}}=9.6 \mathrm{~Hz},{ }^{3} J_{5^{\prime}, 6 a^{\prime}}=4.8 \mathrm{~Hz},{ }^{3} J_{5^{\prime}, 6 b^{\prime}}=2.6 \mathrm{~Hz}, 1 \mathrm{H}, \mathrm{H}_{5^{\prime}}\right), 4.18-4.09\left(\mathrm{~m}, 2 \mathrm{H}, \mathrm{H}_{6 \mathrm{a}^{\prime} / 6 \mathrm{~b}^{\prime}}\right)$ $2.27-2.22\left(\mathrm{~m}, 1 \mathrm{H}, \mathrm{COCH}_{2} \mathrm{CH}\left(\mathrm{CH}_{3}\right)_{2}\right), 2.20-2.14$ (m, 3H, $\left.\mathrm{COCH}_{2} \mathrm{CH}\left(\mathrm{CH}_{3}\right)_{2}\right), 2.10(\mathrm{~d}, J=6.9 \mathrm{~Hz}$, $\left.2 \mathrm{H}, \mathrm{COCH}_{2} \mathrm{CH}\left(\mathrm{CH}_{3}\right)_{2}\right), 1.99-1.84$ (m, 5H, $\left.\mathrm{COCH}_{2} \mathrm{CH}\left(\mathrm{CH}_{3}\right)_{2}\right), 1.68-1.58(\mathrm{~m}, 1 \mathrm{H}$, $\left.\mathrm{COCH}_{2} \mathrm{CH}\left(\mathrm{CH}_{3}\right)_{2}\right), 0.91-0.88\left(\mathrm{~m}, 6 \mathrm{H}, \mathrm{COCH}_{2} \mathrm{CH}\left(\mathrm{CH}_{3}\right)_{2}\right), 0.85(\mathrm{~d}, J=6.6 \mathrm{~Hz}, 12 \mathrm{H}$, $\left.\mathrm{COCH}_{2} \mathrm{CH}\left(\mathrm{CH}_{3}\right)_{2}\right), 0.57$ (d, $\left.J=6.6 \mathrm{~Hz}, 6 \mathrm{H}, \mathrm{COCH}_{2} \mathrm{CH}\left(\mathrm{CH}_{3}\right)_{2}\right)$. Assignments were confirmed by ${ }^{1} \mathrm{H}-$ ${ }^{1} \mathrm{H}$ gCOSY. ${ }^{13} \mathrm{C}$ NMR $\left(500 \mathrm{MHz}, \quad \mathrm{DMSO}-d_{6}\right): \delta=171.8,171.1,171.0,170.3(4 \times$ $\left.\mathrm{COCH}_{2} \mathrm{CH}\left(\mathrm{CH}_{3}\right)_{2}\right), 145.8\left(\mathrm{C}_{1^{\prime \prime} / 4}\right), 143.6\left(\mathrm{C}_{1 " / 4}\right), 133.2\left(\mathrm{C}_{\text {triazole }}\right), 126.5\left(2 \times \mathrm{CH}_{\text {arom }}\right), 125.5(2 \times$ Carom $), 121.7\left(\mathrm{CH}_{\text {triazole }}\right), 84.1\left(\mathrm{C}_{1^{\prime}}\right), 73.4\left(\mathrm{C}_{5^{\prime}}\right), 71.8\left(\mathrm{C}_{3^{\prime}}\right), 70.2\left(\mathrm{C}_{2^{\prime}}\right), 67.4\left(\mathrm{C}_{4^{\prime}}\right), 61.4\left(\mathrm{C}_{6^{\prime}}\right), 42.29$, 42.25, 42.18, $42.0\left(4 \times \mathrm{COCH}_{2} \mathrm{CH}\left(\mathrm{CH}_{3}\right)_{2}\right), 25.1,25.0,24.88,24.85\left(4 \times \mathrm{COCH}_{2} \mathrm{CH}\left(\mathrm{CH}_{3}\right)_{2}\right), 22.01$ (4C), 21.98, 21.95, 21.53, $21.45\left(8 \times \mathrm{COCH}_{2} \mathrm{CH}\left(\mathrm{CH}_{3}\right)_{2}\right)$. Assignments were confirmed by ${ }^{1} \mathrm{H}^{-13} \mathrm{C}$ gHSQC. LRMS $\left(\mathrm{ESI}^{+}\right): \mathrm{m} / \mathrm{z}=745[\mathrm{M}+\mathrm{Na}]^{+}$. HRMS (ESI) calcd for $\mathrm{C}_{34} \mathrm{H}_{20} \mathrm{~N}_{4} \mathrm{O}_{11} \mathrm{SNa}$ 745.3089, found 745.3082 . 


\section{4-[3"'-(Aminosulfonyl)phenyl]-1-(ק-D-glucopyranosyl)-1H-1,2,3-triazole (19)}

The title compound 19 was prepared from triazole 20 (60 mg, $0.11 \mathrm{mmol})$ according to general procedure 2 and isolated as a white solid (41 mg, 98\%). $R_{f}=0.62\left(\mathrm{CH}_{3} \mathrm{CN} / \mathrm{H}_{2} \mathrm{O}\right.$ 9:1). mp 210-213 ${ }^{\circ} \mathrm{C} .[\alpha]^{21}{ }_{\mathrm{D}}-63\left(c=0.12, \mathrm{CH}_{3} \mathrm{OH}\right) .{ }^{1} \mathrm{H}$ NMR $\left(500 \mathrm{MHz}, \mathrm{DMSO}-\mathrm{d}_{6}\right): \delta=8.97\left(\mathrm{~s}, 1 \mathrm{H}, \mathrm{CH}_{\text {triazole }}\right), 8.40$ (bs, $1 \mathrm{H}, \mathrm{H}_{2^{\prime \prime}}$ ), 8.06 (bd, ${ }^{3} J_{4^{\prime \prime} / 6^{\prime \prime}, 5^{\prime \prime}}=7.7 \mathrm{~Hz}, 1 \mathrm{H}, \mathrm{H}_{4^{\prime \prime}}$ or $\left.6^{\prime \prime}\right), 7.87$ (bd, ${ }^{3} J_{4^{\prime \prime} / 6^{\prime \prime}, 5^{\prime \prime}}=7.9 \mathrm{~Hz}, 1 \mathrm{H}, \mathrm{H}_{4^{\prime \prime}}$ or 6"), 7.66 (t, $\left.{ }^{3} J_{5^{\prime \prime}, 4^{\prime \prime} / 6^{\prime \prime}}=7.8 \mathrm{~Hz}, 1 \mathrm{H}, \mathrm{H}_{5^{\prime \prime}}\right), 7.42$ (bs, $2 \mathrm{H}, \mathrm{SO}_{2} \mathrm{NH}_{2}$ ), $5.60\left(\mathrm{~d},{ }^{3} J_{1^{\prime}, 2^{\prime}}=9.3 \mathrm{~Hz}, 1 \mathrm{H}, \mathrm{H}_{1^{\prime}}\right), 5.43(\mathrm{~d}, J=$ $5.9 \mathrm{~Hz}, 1 \mathrm{H}, \mathrm{OH}), 5.31$ (d, $J=4.7 \mathrm{~Hz}, 1 \mathrm{H}, \mathrm{OH}), 5.16(\mathrm{~d}, J=5.4 \mathrm{~Hz}, 1 \mathrm{H}, \mathrm{OH}), 4.61(\mathrm{t}, J=5.6 \mathrm{~Hz}, 1 \mathrm{H}$, OH), $3.84-3.78\left(\mathrm{~m}, 1 \mathrm{H}, \mathrm{H}_{2^{\prime}}\right), 3.75$ - $3.69\left(\mathrm{~m}, 1 \mathrm{H}, \mathrm{H}_{6^{\prime}}\right), 3.52$ - 3.40 (m, 3H, $\left.\mathrm{H}_{3^{\prime}}, \mathrm{H}_{5^{\prime}}, \mathrm{H}_{6^{\prime}}\right), 3.31$ - 3.24 (m, $\left.1 \mathrm{H}, \mathrm{H}_{4}\right)$. Assignments were confirmed by ${ }^{1} \mathrm{H}-{ }^{1} \mathrm{H}$ gCOSY. ${ }^{13} \mathrm{C}$ NMR $\left(500 \mathrm{MHz}\right.$, DMSO- $\left.d_{6}\right): \delta=$ 145.2 ( $\mathrm{C}_{1 "}$ or 3"), 144.9 ( $\mathrm{C}_{1 "}$ or 3"), 131.3 ( $\left.\mathrm{C}_{\text {triazole }}\right), 129.7\left(\mathrm{C}_{5^{\prime \prime}}\right), 128.2$ ( $\mathrm{C}_{4^{\prime \prime}}$ or 6"), $124.9\left(\mathrm{C}_{4^{\prime \prime}}\right.$ or 6"), 122.1 $\left(\mathrm{C}_{2^{\prime \prime}}\right), 121.2\left(\mathrm{CH}_{\text {triazole }}\right), 87.7\left(\mathrm{C}_{1^{\prime}}\right), 79.9\left(\mathrm{C}_{3^{\prime} \text { or } 5^{\prime}}\right), 76.8\left(\mathrm{C}_{3^{\prime} \text { or } 5^{\prime}}\right), 72.2\left(\mathrm{C}_{2^{\prime}}\right), 69.6\left(\mathrm{C}_{4^{\prime}}\right), 60.7\left(\mathrm{C}_{6^{\prime}}\right)$. Assignments were confirmed by ${ }^{1} \mathrm{H}^{13}{ }^{13}$ gHSQC. LRMS $\left(\mathrm{ESI}^{+}\right): \mathrm{m} / \mathrm{z}=409[\mathrm{M}+\mathrm{Na}]^{+}$. HRMS (ESI) calcd for $\mathrm{C}_{14} \mathrm{H}_{18} \mathrm{~N}_{4} \mathrm{O}_{7} \mathrm{SNa}$ 409.0788, found 409.0790.

\section{4-[3"'-(Aminosulfonyl)phenyl]-1-(2',3',4',6'-tetra-O-acetyl- $\beta$-D-glucopyranosyl)-1H-1,2,3-} triazole (20)

The title compound 20 was prepared from glucopyranosyl azide 1 (168 mg, $0.45 \mathrm{mmol}$ ) and alkyne 12 (90 mg, $0.50 \mathrm{mmol}$ ) according to general procedure $1 \mathrm{in} 5 \mathrm{~h}$. Purification of the crude product by flash chromatography (EtOAc/hexane 1:1) afforded 20 (199 mg, 80\%) as a white solid. $R_{f}=0.18$ (EtOAc/Hexane 1:1). mp 103-105 ${ }^{\circ} \mathrm{C} .[\alpha]^{26}{ }_{\mathrm{D}}-57\left(c=0.089, \mathrm{CHCl}_{3}\right) .{ }^{1} \mathrm{H}$ NMR $(500 \mathrm{MHz}$, DMSO$d_{6}$ ): $\delta=9.11$ (s, $1 \mathrm{H}, \mathrm{CH}_{\text {triazole }}$ ), 8.33 (bs, $1 \mathrm{H}, \mathrm{H}_{2^{\prime \prime}}$ ), 8.04 (bd, ${ }^{3} J_{4^{\prime \prime} / 6^{\prime \prime}, 5^{\prime \prime}}=7.8, \mathrm{~Hz}, 1 \mathrm{H}, \mathrm{H}_{4^{\prime \prime}}$ or 6"), 7.82 (bd, ${ }^{3} J_{4^{\prime \prime} / 6^{\prime \prime}, 5^{\prime \prime}}=7.8, \mathrm{~Hz}, 1 \mathrm{H}, \mathrm{H}_{4^{\prime \prime}}$ or 6"), $7.68\left(\mathrm{t},{ }^{3} J_{5^{\prime \prime}, 4^{\prime \prime} 6^{\prime \prime}}=7.8 \mathrm{~Hz}, 1 \mathrm{H}, \mathrm{H}_{5^{\prime \prime}}\right), 7.43$ (bs, $2 \mathrm{H}, \mathrm{SO}_{2} \mathrm{NH}_{2}$ ), 6.42 (d, $\left.{ }^{3} J_{1^{\prime}, 2^{\prime}}=9.0 \mathrm{~Hz}, 1 \mathrm{H}, \mathrm{H}_{1^{\prime}}\right), 5.68\left(\mathrm{dd} \approx \mathrm{t},{ }^{3} J_{3^{\prime}, 2^{\prime}}=9.3 \mathrm{~Hz},{ }^{3} J_{2^{\prime}, 3^{\prime}}=9.0 \mathrm{~Hz}, 1 \mathrm{H}, \mathrm{H}_{2^{\prime}}\right), 5.61\left(\mathrm{t},{ }^{3} J_{3^{\prime}, 2^{\prime}}=9.3 \mathrm{~Hz}\right.$, 
$\left.{ }^{3} J_{3^{\prime}, 4^{\prime}}=9.3 \mathrm{~Hz}, 1 \mathrm{H}, \mathrm{H}_{3^{\prime}}\right), 5.19\left(\mathrm{dd} \approx \mathrm{t},{ }^{3} J_{4^{\prime}, 5^{\prime}}=9.7 \mathrm{~Hz},{ }^{3} J_{4^{\prime}, 3^{\prime}}=9.3 \mathrm{~Hz}, 1 \mathrm{H}, \mathrm{H}_{4^{\prime}}\right), 4.43\left(\mathrm{ddd},{ }^{3} J_{5^{\prime}, 4^{\prime}}=9.7 \mathrm{~Hz}\right.$, $\left.{ }^{3} J_{5^{\prime}, 6 a^{\prime}}=5.4 \mathrm{~Hz},{ }^{3} J_{5^{\prime}, 6 b^{\prime}}=1.7 \mathrm{~Hz}, 1 \mathrm{H}, \mathrm{H}_{5^{\prime}}\right), 4.18\left(\mathrm{dd},{ }^{2} J_{6 a^{\prime}, 6 b^{\prime}}=12.6 \mathrm{~Hz},{ }^{3} J_{6 a^{\prime}, 5^{\prime}}=5.4 \mathrm{~Hz}, 1 \mathrm{H}, \mathrm{H}_{6 \mathrm{a}^{\prime}}\right), 4.11$ (dd, ${ }^{2} J_{6 b^{\prime}, 6 a^{\prime}}=12.6 \mathrm{~Hz},{ }^{3} J_{6 b^{\prime}, 5^{\prime}}=1.7 \mathrm{~Hz}, 1 \mathrm{H}, \mathrm{H}_{6 b^{\prime}}$ ), 2.04 (s, 3H, $\mathrm{COCH}_{3}$ ), 2.01 (s, 3H, $\mathrm{COCH}_{3}$ ), 1.98 (s, 3H, $\mathrm{COCH}_{3}$ ), 1.81 (s, 3H, $\mathrm{COCH}_{3}$ ). Assignments were confirmed by ${ }^{1} \mathrm{H}-{ }^{1} \mathrm{H}$ gCOSY. ${ }^{13} \mathrm{C}$ NMR (500 MHz, DMSO- $\left.d_{6}\right): \delta=170.0,169.5,169.4,168.6\left(4 \times \mathrm{COCH}_{3}\right), 145.8$ ( $\left.\mathrm{C}_{1 \text { " or 3" }}\right), 145.0\left(\mathrm{C}_{1^{\prime \prime} \text { or 3" }}\right), 130.7$ ( $\left.\mathrm{C}_{\text {triazole }}\right), 129.9\left(\mathrm{C}_{5^{\prime \prime}}\right), 128.3\left(\mathrm{C}_{4^{\prime \prime}}\right.$ or 6"), $125.3\left(\mathrm{C}_{4^{\prime \prime}}\right.$ or 6" $), 122.2\left(\mathrm{C}_{2^{\prime \prime}}\right), 121.2\left(\mathrm{CH}_{\text {triazole }}\right), 84.1\left(\mathrm{C}_{1}\right), 73.3$ $\left(\mathrm{C}_{5^{\prime}}\right), 72.0\left(\mathrm{C}_{3^{\prime}}\right), 70.4\left(\mathrm{C}_{2^{\prime}}\right), 67.6\left(\mathrm{C}_{4^{\prime}}\right), 61.8\left(\mathrm{C}_{6^{\prime}}\right), 20.5\left(\mathrm{COCH}_{3}\right), 20.4\left(\mathrm{COCH}_{3}\right), 20.2\left(\mathrm{COCH}_{3}\right), 19.9$ $\left(\mathrm{COCH}_{3}\right)$. Assignments were confirmed by ${ }^{1} \mathrm{H}^{-13} \mathrm{C}$ gHSQC. LRMS $\left(\mathrm{ESI}^{+}\right): \mathrm{m} / \mathrm{z}=555[\mathrm{M}+\mathrm{H}]^{+}, 577$ $[\mathrm{M}+\mathrm{Na}]^{+}$. HRMS (ESI) calcd for $\mathrm{C}_{22} \mathrm{H}_{26} \mathrm{~N}_{4} \mathrm{O}_{11} \mathrm{SNa}$ 577.1211, found 57.1235.

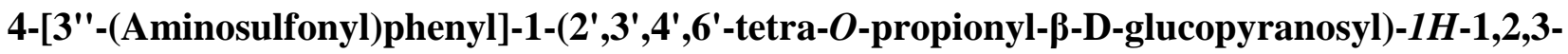

\section{triazole (21)}

The title compound 21 was prepared from glucopyranosyl azide 2 (123 mg, $0.29 \mathrm{mmol})$ and alkyne 12 (57 mg, $0.31 \mathrm{mmol}$ ) according to general procedure 1 in $5.5 \mathrm{~h}$. Purification of the crude product by flash chromatography (EtOAc/hexane 2:3) afforded 21 (118 mg, 67\%) as a white solid. $R_{f}=0.13$ (EtOAc/Hexane 2:3). mp $78-85{ }^{\circ} \mathrm{C}$ (dec.). $[\alpha]^{26}-42\left(c=0.067, \mathrm{CHCl}_{3}\right) .{ }^{1} \mathrm{H}$ NMR $(500 \mathrm{MHz}$, DMSO- $d_{6}$ ): $\delta=9.13$ (s, $1 \mathrm{H}, \mathrm{CH}_{\text {triazole }}$ ), 8.33 (bs, $1 \mathrm{H}, \mathrm{H}_{2^{\prime \prime}}$ ), 8.03 (bd, ${ }^{3} J_{4^{\prime \prime} / 6^{\prime \prime} 5^{\prime \prime}}=7.8 \mathrm{~Hz}, 1 \mathrm{H}, \mathrm{H}_{4^{\prime \prime}}$ or $6^{\prime \prime}$ ), $7.82\left(\mathrm{bd},{ }^{3} J_{4^{\prime \prime} / 6^{\prime \prime}, 5^{\prime \prime}}=7.8 \mathrm{~Hz}, 1 \mathrm{H}, \mathrm{H}_{4^{\prime \prime}}\right.$ or 6") $7.68\left(\mathrm{t},{ }^{3} J_{5^{\prime \prime}, 4^{\prime \prime} / 6^{\prime \prime}}=7.8 \mathrm{~Hz}, 1 \mathrm{H}, \mathrm{H}_{5^{\prime \prime}}\right), 7.43$ (bs, $2 \mathrm{H}, \mathrm{SO}_{2} \mathrm{NH}_{2}$ ), $6.44\left(\mathrm{~d},{ }^{3} J_{1^{\prime}, 2^{\prime}}=9.1 \mathrm{~Hz}, 1 \mathrm{H}, \mathrm{H}_{1^{\prime}}\right), 5.72\left(\mathrm{dd} \approx \mathrm{t},{ }^{3} J_{2^{\prime}, 3^{\prime}}=9.3 \mathrm{~Hz},{ }^{3} J_{2^{\prime}, 3^{\prime}}=9.1 \mathrm{~Hz}, 1 \mathrm{H}, \mathrm{H}_{2^{\prime}}\right), 5.64\left(\mathrm{dd} \approx \mathrm{t},{ }^{3} J_{3^{\prime}, 2^{\prime}}\right.$ $\left.=9.3 \mathrm{~Hz},{ }^{3} J_{3^{\prime}, 4^{\prime}}=9.5 \mathrm{~Hz}, 1 \mathrm{H}, \mathrm{H}_{3^{\prime}}\right), 5.23\left(\mathrm{dd} \approx \mathrm{t},{ }^{3} J_{4^{\prime}, 5^{\prime}}=9.7 \mathrm{~Hz},{ }^{3} J_{4^{\prime}, 3^{\prime}}=9.5 \mathrm{~Hz}, 1 \mathrm{H}, \mathrm{H}_{4^{\prime}}\right), 4.45\left(\mathrm{ddd},{ }^{3} J_{5^{\prime}, 4^{\prime}}\right.$ $\left.=9.7 \mathrm{~Hz},{ }^{3} J_{5^{\prime}, 6 a^{\prime}}=5.3 \mathrm{~Hz},{ }^{3} J_{5^{\prime}, 6 b^{\prime}}=1.7 \mathrm{~Hz}, 1 \mathrm{H}, \mathrm{H}_{5^{\prime}}\right), 4.22\left(\mathrm{dd},{ }^{2} J_{6 a^{\prime}, 6 b^{\prime}}=12.6 \mathrm{~Hz},{ }^{3} J_{6 a^{\prime}, 5^{\prime}}=5.3 \mathrm{~Hz}, 1 \mathrm{H}\right.$, $\left.\mathrm{H}_{6 \mathrm{a}^{\prime}}\right), 4.12\left(\mathrm{dd},{ }^{2} \mathrm{~J}_{6 b^{\prime}, 6 \mathrm{a}^{\prime}}=12.6 \mathrm{~Hz},{ }^{3} \mathrm{~J}_{6 b^{\prime}, 5^{\prime}}=1.7 \mathrm{~Hz}, 1 \mathrm{H}, \mathrm{H}_{6 \mathrm{~b}^{\prime}}\right), 2.40-2.27\left(\mathrm{~m}, 4 \mathrm{H}, \mathrm{COCH}_{2} \mathrm{CH}_{3}\right), 2.26-$ 2.20 (m, 2H, $\mathrm{COCH}_{2} \mathrm{CH}_{3}$ ), $2.13-2.01\left(\mathrm{~m}, 2 \mathrm{H}, \mathrm{COCH}_{2} \mathrm{CH}_{3}\right), 1.04-0.95$ (m, 9H, $\left.\mathrm{COCH}_{2} \mathrm{CH}_{3}\right), 0.78$ (t, $J=7.5 \mathrm{~Hz}, 3 \mathrm{H}, \mathrm{COCH}_{2} \mathrm{CH}_{3}$ ). Assignments were confirmed by ${ }^{1} \mathrm{H}-{ }^{1} \mathrm{H}$ gCOSY. ${ }^{13} \mathrm{C}$ NMR (500 
MHz, DMSO- $\left.d_{6}\right): \delta=173.3,172.8,172.6,172.0\left(4 \times \mathrm{COCH}_{2} \mathrm{CH}_{3}\right), 145.8\left(\mathrm{C}_{1 "}\right.$ or 3"), $145.0\left(\mathrm{C}_{1 "}\right.$ or 3"), 130.7 ( $\left.\mathrm{C}_{\text {triazole }}\right), 129.9\left(\mathrm{C}_{5 "}\right), 128.3\left(\mathrm{C}_{4 " \text { or } 6 "}{ }^{\prime \prime}\right), 125.3\left(\mathrm{C}_{4 " \text { or } 6 "}\right), 122.1\left(\mathrm{C}_{2 "}\right), 121.2\left(\mathrm{CH}_{\text {triazole }}\right), 84.1\left(\mathrm{C}_{1^{\prime}}\right)$, $73.4\left(\mathrm{C}_{5^{\prime}}\right), 72.0\left(\mathrm{C}_{3^{\prime}}\right), 70.3\left(\mathrm{C}_{2^{\prime}}\right), 67.3\left(\mathrm{C}_{4^{\prime}}\right), 61.5\left(\mathrm{C}_{6^{\prime}}\right)$, 26.8, 26.7, 26.6, $26.4\left(4 \times \mathrm{COCH}_{2} \mathrm{CH}_{3}\right), 8.91$, 8.88, 8.86, $8.8\left(4 \times \mathrm{COCH}_{2} \mathrm{CH}_{3}\right)$. Assignments were confirmed by ${ }^{1} \mathrm{H}^{13}{ }^{13}$ gHSQC. LRMS $\left(\mathrm{ESI}^{+}\right)$: $m / z=611[\mathrm{M}+\mathrm{H}]^{+} . \mathrm{HRMS}(\mathrm{ESI})$ calcd for $\mathrm{C}_{26} \mathrm{H}_{34} \mathrm{~N}_{4} \mathrm{O}_{11} \mathrm{SNa}$ 633.1837, found 633.1826.

\section{4-[3' '-(Aminosulfonyl)phenyl]-1-(2',3',4',6'-tetra-O-butyryl- $\beta$-D-glucopyranosyl)-1H-1,2,3-} triazole (22)

The title compound 22 was prepared from glucopyranosyl azide 3 (74 mg, 0.15 mmol) and alkyne 12 (30 mg, $0.17 \mathrm{mmol}$ ) according to general procedure 1 in 1 day. Purification of the crude product by flash chromatography (EtOAc/hexane 2:3) afforded 22 (80 mg, 80\%) as a white solid. $R_{f}=0.24$ (EtOAc/hexane 2:3). mp 57-75 ${ }^{\circ} \mathrm{C}$ (dec.). $[\alpha]^{25}{ }_{\mathrm{D}}-59\left(c=0.056, \mathrm{CHCl}_{3}\right) .{ }^{1} \mathrm{H}$ NMR (500 $\mathrm{MHz}$, DMSO-d $\left.)_{6}\right): \delta=9.12\left(\mathrm{~s}, 1 \mathrm{H}, \mathrm{CH}_{\text {triazole }}\right.$ ), 8.32 (bs, $1 \mathrm{H}, \mathrm{H}_{2 "}$ ), 8.02 (bd, ${ }^{3} J_{4^{\prime \prime} / 6^{\prime \prime}, 5^{\prime \prime}}=7.8, \mathrm{~Hz}, 1 \mathrm{H}, \mathrm{H}_{4 "}$ or $\left.6^{\prime \prime}\right)$, $7.82\left(\mathrm{bd},{ }^{3} J_{4^{\prime \prime} / 6^{\prime \prime}, 5^{\prime \prime}}=7.8 \mathrm{~Hz}, 1 \mathrm{H}, \mathrm{H}_{4 "}\right.$ or $\left.6^{\prime \prime}\right), 7.68$ (t, $\left.{ }^{3} J_{5^{\prime \prime}, 4^{\prime \prime} / 6^{\prime \prime}}=7.8 \mathrm{~Hz}, 1 \mathrm{H}, \mathrm{H}_{5 "}\right), 7.44$ (bs, $2 \mathrm{H}, \mathrm{SO}_{2} \mathrm{NH}_{2}$ ), $6.43\left(\mathrm{~d},{ }^{3} J_{1^{\prime}, 2^{\prime}}=9.1 \mathrm{~Hz}, 1 \mathrm{H}, \mathrm{H}_{1^{\prime}}\right), 5.73\left(\mathrm{dd} \approx \mathrm{t},{ }^{3} J_{2^{\prime}, 3^{\prime}}=9.3 \mathrm{~Hz},{ }^{3} J_{2^{\prime}, 3^{\prime}}=9.1 \mathrm{~Hz}, 1 \mathrm{H}, \mathrm{H}_{2^{\prime}}\right), 5.66\left(\mathrm{dd} \approx \mathrm{t},{ }^{3} J_{3^{\prime}, 2^{\prime}}\right.$ $\left.=9.3 \mathrm{~Hz},{ }^{3} J_{3^{\prime}, 4^{\prime}}=9.5 \mathrm{~Hz}, 1 \mathrm{H}, \mathrm{H}_{3^{\prime}}\right), 5.24\left(\mathrm{dd} \approx \mathrm{t},{ }^{3} J_{4^{\prime}, 5^{\prime}}=9.7 \mathrm{~Hz},{ }^{3} J_{4^{\prime}, 3^{\prime}}=9.5 \mathrm{~Hz}, 1 \mathrm{H}, \mathrm{H}_{4^{\prime}}\right.$ ), 4.45 (ddd, $\left.{ }^{3} J_{5^{\prime}, 4^{\prime}}=9.7 \mathrm{~Hz},{ }^{3} J_{5^{\prime}, 6 a^{\prime}}=5.2 \mathrm{~Hz},{ }^{3} J_{5^{\prime}, 6 b^{\prime}}=2.0 \mathrm{~Hz}, 1 \mathrm{H}, \mathrm{H}_{5^{\prime}}\right), 4.18\left(\mathrm{dd},{ }^{2} J_{6 a^{\prime}, 6 b^{\prime}}=12.6 \mathrm{~Hz},{ }^{3} J_{6 a^{\prime}, 5^{\prime}}=5.2 \mathrm{~Hz}\right.$, $\left.1 \mathrm{H}, \mathrm{H}_{6 \mathrm{a}^{\prime}}\right), 4.13\left(\mathrm{dd},{ }^{2} J_{6 b^{\prime}, 6 a^{\prime}}=12.6 \mathrm{~Hz},{ }^{3} J_{6 b^{\prime}, 5^{\prime}}=2.0 \mathrm{~Hz}, 1 \mathrm{H}, \mathrm{H}_{6 b^{\prime}}\right), 2.36-2.21(\mathrm{~m}, 4 \mathrm{H}$, $\mathrm{COCH}_{2} \mathrm{CH}_{2} \mathrm{CH}_{3}$ ), 2.19 (t, $J=7.2 \mathrm{~Hz}, 2 \mathrm{H}, \mathrm{COCH}_{2} \mathrm{CH}_{2} \mathrm{CH}_{3}$ ), $2.06-2.01$ (m, 2H, $\mathrm{COCH}_{2} \mathrm{CH}_{2} \mathrm{CH}_{3}$ ), $1.57-1.43\left(\mathrm{~m}, 6 \mathrm{H}, \mathrm{COCH}_{2} \mathrm{CH}_{2} \mathrm{CH}_{3}\right), 1.32$ - 1.20 (m, 2H, $\left.\mathrm{COCH}_{2} \mathrm{CH}_{2} \mathrm{CH}_{3}\right), 0.90-0.80(\mathrm{~m}, 9 \mathrm{H}$, $\mathrm{COCH}_{2} \mathrm{CH}_{2} \mathrm{CH}_{3}$ ), 0.57 (t, $J=7.4 \mathrm{~Hz}, 3 \mathrm{H}, \mathrm{COCH}_{2} \mathrm{CH}_{2} \mathrm{CH}_{3}$ ). Assignments were confirmed by ${ }^{1} \mathrm{H}^{-1} \mathrm{H}$ gCOSY. ${ }^{13} \mathrm{C}$ NMR (500 MHz, DMSO-d $): \delta=172.4,171.8,171.6,170.9\left(4 \times \mathrm{COCH}_{2} \mathrm{CH}_{2} \mathrm{CH}_{3}\right)$, $145.8\left(\mathrm{C}_{1 "}\right.$ or 3"), $145.0\left(\mathrm{C}_{1 "}\right.$ or 3"), 130.7 ( $\left.\mathrm{C}_{\text {triazole }}\right), 129.8\left(\mathrm{C}_{5 "}\right), 128.2\left(\mathrm{C}_{4 "}\right.$ or 6"), $125.3\left(\mathrm{C}_{4 " \text { or 6" }}\right), 122.1$ $\left(\mathrm{C}_{2^{\prime \prime}}\right), 121.2\left(\mathrm{CH}_{\text {triazole }}\right), 84.1\left(\mathrm{C}_{1^{\prime}}\right), 73.4\left(\mathrm{C}_{5^{\prime}}\right), 71.8\left(\mathrm{C}_{3^{\prime}}\right), 70.2\left(\mathrm{C}_{2^{\prime}}\right), 67.3\left(\mathrm{C}_{4^{\prime}}\right), 61.4\left(\mathrm{C}_{6^{\prime}}\right)$, 35.14, 35.08, 
35.06, $34.8\left(4 \times \mathrm{COCH}_{2} \mathrm{CH}_{2} \mathrm{CH}_{3}\right), 17.70$ (2C), 17.67 (2C) $\left(4 \times \mathrm{COCH}_{2} \mathrm{CH}_{2} \mathrm{CH}_{3}\right), 13.27,13.26,13.2$, $12.8\left(4 \times \mathrm{COCH}_{2} \mathrm{CH}_{2} \mathrm{CH}_{3}\right)$. Assignments were confirmed by ${ }^{1} \mathrm{H}^{-13} \mathrm{C}$ gHSQC. LRMS $\left(\mathrm{ESI}^{+}\right): \mathrm{m} / \mathrm{z}=$ $689[\mathrm{M}+\mathrm{Na}]^{+}$. HRMS (ESI) calcd for $\mathrm{C}_{30} \mathrm{H}_{42} \mathrm{~N}_{4} \mathrm{O}_{11} \mathrm{SNa}$ 689.2463, found 689.2482.

\section{4-[3"-(Aminosulfonyl)phenyl]-1-(2',3',4',6'-tetra-O-pentyryl-p-D-glucopyranosyl)-1H-1,2,3-}

\section{triazole (23)}

The title compound 23 was prepared from glucopyranosyl azide 4 (142 mg, $0.260 \mathrm{mmol}$ ) and alkyne 12 (81 mg, $0.45 \mathrm{mmol}$ ) according to general procedure $1 \mathrm{in} 4 \mathrm{~h}$. Purification of the crude product by flash chromatography $\left(\mathrm{CH}_{2} \mathrm{Cl}_{2}\right.$ to $\mathrm{CH}_{2} \mathrm{Cl}_{2} / \mathrm{MeOH}$ 99:1) afforded 23 (104 mg, 55\%) as a white solid. $R_{f}=0.26\left(\mathrm{CH}_{2} \mathrm{Cl}_{2} / \mathrm{MeOH} 99: 1\right) . \mathrm{mp} 107-111^{\circ} \mathrm{C} \cdot[\alpha]^{25}{ }_{\mathrm{D}}-34\left(c=0.056, \mathrm{CHCl}_{3}\right) .{ }^{1} \mathrm{H}$ NMR $(500 \mathrm{MHz}$,

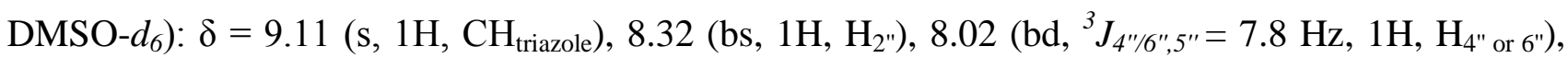

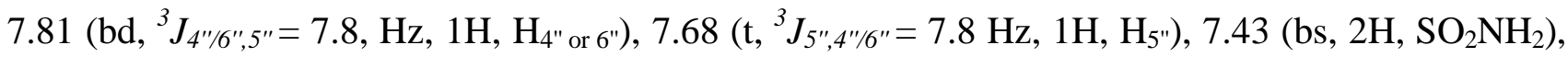
$6.43\left(\mathrm{~d},{ }^{3} J_{1^{\prime}, 2^{\prime}}=9.1 \mathrm{~Hz}, 1 \mathrm{H}, \mathrm{H}_{1^{\prime}}\right), 5.72\left(\mathrm{dd} \approx \mathrm{t},{ }^{3} J_{2^{\prime}, 3^{\prime}}=9.3 \mathrm{~Hz},{ }^{3} J_{2^{\prime}, 3^{\prime}}=9.1 \mathrm{~Hz}, 1 \mathrm{H}, \mathrm{H}_{2^{\prime}}\right), 5.65\left(\mathrm{dd} \approx \mathrm{t},{ }^{3} J_{3^{\prime}, 2^{\prime}}\right.$ $\left.=9.3 \mathrm{~Hz},{ }^{3} J_{3^{\prime}, 4^{\prime}}=9.5 \mathrm{~Hz}, 1 \mathrm{H}, \mathrm{H}_{3^{\prime}}\right), 5.23\left(\mathrm{dd} \approx \mathrm{t},{ }^{3} J_{4^{\prime}, 5^{\prime}}=9.7 \mathrm{~Hz},{ }^{3} J_{4^{\prime}, 3^{\prime}}=9.5 \mathrm{~Hz}, 1 \mathrm{H}, \mathrm{H}_{4^{\prime}}\right), 4.44\left(\mathrm{ddd},{ }^{3} J_{5^{\prime}, 4^{\prime}}\right.$ $\left.=9.7 \mathrm{~Hz},{ }^{3} J_{5^{\prime}, 6 a^{\prime}}=5.1 \mathrm{~Hz},{ }^{3} J_{5^{\prime}, 6 b^{\prime}}=2.1 \mathrm{~Hz}, 1 \mathrm{H}, \mathrm{H}_{5^{\prime}}\right), 4.17\left(\mathrm{dd},{ }^{2} J_{6 a^{\prime}, 6 b^{\prime}}=12.6 \mathrm{~Hz},{ }^{3} J_{6 a^{\prime}, 5^{\prime}}=5.1 \mathrm{~Hz}, 1 \mathrm{H}\right.$, $\mathrm{H}_{6 \mathrm{a}^{\prime}}$ ), $4.12\left(\mathrm{dd},{ }^{2} J_{6 b^{\prime}, 6 a^{\prime}}=12.6 \mathrm{~Hz},{ }^{3} J_{6 b^{\prime}, 5^{\prime}}=2.1 \mathrm{~Hz}, 1 \mathrm{H}, \mathrm{H}_{6 \mathrm{~b}^{\prime}}\right), 2.38-2.23(\mathrm{~m}, 4 \mathrm{H}$, $\left.\mathrm{COCH}_{2} \mathrm{CH}_{2} \mathrm{CH}_{2} \mathrm{CH}_{3}\right), 2.21\left(\mathrm{t}, J=7.4 \mathrm{~Hz}, 2 \mathrm{H}, \mathrm{COCH}_{2} \mathrm{CH}_{2} \mathrm{CH}_{2} \mathrm{CH}_{3}\right), 2.08-2.01(\mathrm{~m}, 2 \mathrm{H}$, $\left.\mathrm{COCH}_{2} \mathrm{CH}_{2} \mathrm{CH}_{2} \mathrm{CH}_{3}\right), 1.52-1.40\left(\mathrm{~m}, 6 \mathrm{H}, \mathrm{COCH}_{2} \mathrm{CH}_{2} \mathrm{CH}_{2} \mathrm{CH}_{3}\right), 1.30-1.17(\mathrm{~m}, 8 \mathrm{H}$, $\left.\mathrm{COCH}_{2} \mathrm{CH}_{2} \mathrm{CH}_{2} \mathrm{CH}_{3}\right), \quad 0.99-0.89\left(\mathrm{~m}, 2 \mathrm{H}, \mathrm{COCH}_{2} \mathrm{CH}_{2} \mathrm{CH}_{2} \mathrm{CH}_{3}\right) 0.88-0.79$ (m, 9H, $\mathrm{COCH}_{2} \mathrm{CH}_{2} \mathrm{CH}_{2} \mathrm{CH}_{3}$ ), 0.64 (t, $J=7.3 \mathrm{~Hz}, 3 \mathrm{H}, \mathrm{COCH}_{2} \mathrm{CH}_{2} \mathrm{CH}_{2} \mathrm{CH}_{3}$ ). Assignments were confirmed by ${ }^{1} \mathrm{H}-{ }^{1} \mathrm{H}$ gCOSY. ${ }^{13} \mathrm{C}$ NMR (500 MHz, DMSO- $\left.d_{6}\right): \delta=172.5,171.9,171.7,171.1(4 \times$ $\left.\mathrm{COCH}_{2} \mathrm{CH}_{2} \mathrm{CH}_{2} \mathrm{CH}_{3}\right), 145.8$ ( $\left.\mathrm{C}_{1 " \text { or 3") }}\right), 145.0$ ( $\left.\mathrm{C}_{1 " \text { or 3") }}\right) 130.7$ ( $\left.\mathrm{C}_{\text {triazole }}\right), 129.8\left(\mathrm{C}_{5}\right.$ "), $128.2\left(\mathrm{C}_{4 " \text { or 6" }}\right.$ ), $125.3\left(\mathrm{C}_{4^{\prime \prime}}\right.$ or 6" (n), $^{\prime} 122.1\left(\mathrm{C}_{2^{\prime \prime}}\right), 121.1\left(\mathrm{CH}_{\text {triazole }}\right), 84.1\left(\mathrm{C}_{1^{\prime}}\right), 73.4\left(\mathrm{C}_{5^{\prime}}\right), 71.9\left(\mathrm{C}_{3^{\prime}}\right), 70.2\left(\mathrm{C}_{2^{\prime}}\right), 67.4\left(\mathrm{C}_{4^{\prime}}\right)$, $61.5\left(\mathrm{C}_{6}\right), 33.0,32.93,32.91,32.7\left(4 \times \mathrm{COCH}_{2} \mathrm{CH}_{2} \mathrm{CH}_{2} \mathrm{CH}_{3}\right), 26.32,26.29$ (2C), $26.28(4 \times$ 
$\mathrm{COCH}_{2} \mathrm{CH}_{2} \mathrm{CH}_{2} \mathrm{CH}_{3}$ ), 21.48, 21.45 (2C), 21.1 (4 $\left.\times \mathrm{COCH}_{2} \mathrm{CH}_{2} \mathrm{CH}_{2} \mathrm{CH}_{3}\right), 13.49,13.47,13.4,13.3$ (4 $\times \mathrm{COCH}_{2} \mathrm{CH}_{2} \mathrm{CH}_{2} \mathrm{CH}_{3}$ ). Assignments were confirmed by ${ }^{1} \mathrm{H}^{-13} \mathrm{C}$ gHSQC. LRMS (ESI $\left.{ }^{+}\right): m / z=723$ $[\mathrm{M}+\mathrm{H}]^{+}$. HRMS (ESI) calcd for $\mathrm{C}_{34} \mathrm{H}_{50} \mathrm{~N}_{4} \mathrm{O}_{11} \mathrm{SNa}$ 745.3089, found 745.3106 .

\section{4-[3"'-(Aminosulfonyl)phenyl]-1-(2',3',4',6'-tetra-O-(3-methylbutyryl)- $\beta$-D-glucopyranosyl)-1H-}

\section{1,2,3-triazole (24)}

The title compound 24 was prepared from glucopyranosyl azide 5 (260 mg, $0.48 \mathrm{mmol}$ ) and alkyne 12 (96 mg, $0.53 \mathrm{mmol}$ ) according to general procedure 1 in $7.5 \mathrm{~h}$. Purification of the crude product by flash chromatography (EtoAc/hexane 3:7) afforded 24 (258 mg, 74\%) as a white solid. $R_{f}=0.28$ (EtoAc/hexane 2:3). mp 152-153 ${ }^{\circ} \mathrm{C} .[\alpha]^{25} \mathrm{D}-46\left(c=0.072, \mathrm{CHCl}_{3}\right) .{ }^{1} \mathrm{H}$ NMR $(500 \mathrm{MHz}$, DMSO$d_{6}$ ): $\delta=9.11\left(\mathrm{~s}, 1 \mathrm{H}, \mathrm{CH}_{\text {triazole }}\right.$ ), 8.31 (bs, $1 \mathrm{H}, \mathrm{H}_{2^{\prime \prime}}$ ), 8.00 (bd, ${ }^{3} J_{4^{\prime \prime} / 6^{\prime \prime}, 5^{\prime \prime}}=7.8, \mathrm{~Hz}, 1 \mathrm{H}, \mathrm{H}_{4^{\prime \prime}}$ or $\left.6^{\prime \prime}\right), 7.82$ (bd, ${ }^{3} J_{4^{\prime \prime} / 6^{\prime \prime}, 5^{\prime \prime}}=7.8 \mathrm{~Hz}, 1 \mathrm{H}, \mathrm{H}_{4^{\prime \prime}}$ or $\left.6^{\prime \prime}\right), 7.68\left(\mathrm{t},{ }^{3} J_{5^{\prime \prime}, 4^{\prime \prime} / 6^{\prime \prime}}=7.8 \mathrm{~Hz}, 1 \mathrm{H}, \mathrm{H}_{5^{\prime \prime}}\right), 7.44$ (bs, $2 \mathrm{H}, \mathrm{SO}_{2} \mathrm{NH}_{2}$ ), 6.43 (d, $\left.{ }^{3} J_{1^{\prime}, 2^{\prime}}=9.1 \mathrm{~Hz}, 1 \mathrm{H}, \mathrm{H}_{1^{\prime}}\right), 5.74\left(\mathrm{dd} \approx \mathrm{t},{ }^{3} J_{2^{\prime}, 3^{\prime}}=9.3 \mathrm{~Hz},{ }^{3} J_{2^{\prime}, 3^{\prime}}=9.1 \mathrm{~Hz}, 1 \mathrm{H}, \mathrm{H}_{2^{\prime}}\right), 5.67\left(\mathrm{dd} \approx \mathrm{t},{ }^{3} J_{3^{\prime}, 2^{\prime}}=9.3\right.$ $\left.\mathrm{Hz},{ }^{3} J_{3^{\prime}, 4^{\prime}}=9.4 \mathrm{~Hz}, 1 \mathrm{H}, \mathrm{H}_{3^{\prime}}\right), 5.24\left(\mathrm{dd} \approx \mathrm{t},{ }^{3} J_{4^{\prime}, 5^{\prime}}=9.7 \mathrm{~Hz},{ }^{3} J_{4^{\prime}, 3^{\prime}}=9.4 \mathrm{~Hz}, 1 \mathrm{H}, \mathrm{H}_{4^{\prime}}\right), 4.44$ (ddd, ${ }^{3} J_{5^{\prime}, 4^{\prime}}=$

$\left.9.7 \mathrm{~Hz},{ }^{3} J_{5^{\prime}, 6 a^{\prime}}=4.7 \mathrm{~Hz},{ }^{3} J_{5^{\prime}, 6 b^{\prime}}=2.9 \mathrm{~Hz}, 1 \mathrm{H}, \mathrm{H}_{5^{\prime}}\right), 4.18-4.10\left(\mathrm{~m}, 2 \mathrm{H}, \mathrm{H}_{6 \mathrm{a}^{\prime} / 6 b^{\prime}}\right), 2.28-2.21(\mathrm{~m}, 1 \mathrm{H}$, $\left.\mathrm{COCH}_{2} \mathrm{CH}\left(\mathrm{CH}_{3}\right)_{2}\right), 2.20-2.13\left(\mathrm{~m}, 3 \mathrm{H}, \mathrm{COCH}_{2} \mathrm{CH}\left(\mathrm{CH}_{3}\right)_{2}\right), 2.10(\mathrm{~d}, J=6.9 \mathrm{~Hz}, 2 \mathrm{H}$, $\left.\mathrm{COCH}_{2} \mathrm{CH}\left(\mathrm{CH}_{3}\right)_{2}\right), 2.00-1.85$ (m, 5H, $\left.\mathrm{COCH}_{2} \mathrm{CH}\left(\mathrm{CH}_{3}\right)_{2}\right), 1.68-1.60$ (m, $\left.1 \mathrm{H}, \mathrm{COCH}_{2} \mathrm{CH}\left(\mathrm{CH}_{3}\right)_{2}\right)$, $0.92-0.88\left(\mathrm{~m}, 6 \mathrm{H}, \mathrm{COCH}_{2} \mathrm{CH}\left(\mathrm{CH}_{3}\right)_{2}\right), 0.85\left(\mathrm{~d}, J=6.6 \mathrm{~Hz}, 12 \mathrm{H}, \mathrm{COCH}_{2} \mathrm{CH}\left(\mathrm{CH}_{3}\right)_{2}\right), 0.58(\mathrm{~d}, J=6.6$ $\left.\mathrm{Hz}, 6 \mathrm{H}, \mathrm{COCH}_{2} \mathrm{CH}\left(\mathrm{CH}_{3}\right)_{2}\right)$. Assignments were confirmed by ${ }^{1} \mathrm{H}-{ }^{1} \mathrm{H}$ gCOSY.${ }^{13} \mathrm{C}$ NMR (500 MHz, DMSO-d $\left.d_{6}\right): \delta=171.8,171.1,171.0,170.3\left(4 \times \mathrm{COCH}_{2} \mathrm{CH}\left(\mathrm{CH}_{3}\right)_{2}\right), 145.8\left(\mathrm{C}_{1 " \text { or 3" }}\right), 145.0\left(\mathrm{C}_{1 " \text { or } 3^{\prime \prime}}\right)$, 130.7 ( $\left.\mathrm{C}_{\text {triazole }}\right), 129.8\left(\mathrm{C}_{5^{\prime \prime}}\right), 128.2\left(\mathrm{C}_{4^{\prime \prime}}\right.$ or 6" $), 125.3\left(\mathrm{C}_{4^{\prime \prime}}\right.$ or 6"), $122.1\left(\mathrm{C}_{2^{\prime \prime}}\right), 121.2\left(\mathrm{CH}_{\text {triazole }}\right), 84.1\left(\mathrm{C}_{1^{\prime}}\right)$, $73.4\left(\mathrm{C}_{5^{\prime}}\right), \quad 71.7\left(\mathrm{C}_{3^{\prime}}\right), \quad 70.2\left(\mathrm{C}_{2^{\prime}}\right), 67.4\left(\mathrm{C}_{4^{\prime}}\right), 61.4\left(\mathrm{C}_{6^{\prime}}\right), 42.3,42.23,42.15,42.2(4 \times$ $\left.\mathrm{COCH}_{2} \mathrm{CH}\left(\mathrm{CH}_{3}\right)_{2}\right), 25.02,24.97,24.84,24.81\left(4 \times \mathrm{COCH}_{2} \mathrm{CH}\left(\mathrm{CH}_{3}\right)_{2}\right), 21.98$ (2C), 21.97 (2C), 
21.95, 21.9, 21.53, $21.47\left(8 \times \mathrm{COCH}_{2} \mathrm{CH}\left(\mathrm{CH}_{3}\right)_{2}\right)$. Assignments were confirmed by ${ }^{1} \mathrm{H}-{ }^{13} \mathrm{C}$ gHSQC. LRMS $\left(\mathrm{ESI}^{+}\right): \mathrm{m} / \mathrm{z}=723[\mathrm{M}+\mathrm{H}]^{+}, 745[\mathrm{M}+\mathrm{Na}]^{+}$. HRMS (ESI) calcd for $\mathrm{C}_{34} \mathrm{H}_{50} \mathrm{~N}_{4} \mathrm{O}_{11} \mathrm{SNa}$ 745.3089, found 745.3065 .

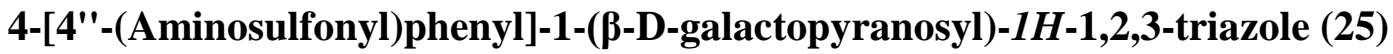

The title compound 25 was prepared from compound 26 (86 mg, $0.16 \mathrm{mmol}$ ) according to general procedure 2 and isolated as a white solid (60 mg, 98\%). $R_{f}=0.58\left(\mathrm{CH}_{3} \mathrm{CN} / \mathrm{H}_{2} \mathrm{O} 9: 1\right) .{ }^{1} \mathrm{H}$ NMR was in agreement with the data reported in the literature. ${ }^{5 \mathrm{~h}}$

\section{4-[4' '-(Aminosulfonyl)phenyl]-1-(2',3',4',6'-tetra-O-acetyl- $\beta$-D-galactopyranosyl)-1H-1,2,3-}

\section{triazole (26)}

The title compound 26 was prepared from galactopyranosyl azide 6 (168 mg, 0.45 mmol) and alkyne 11 (90 mg, $0.50 \mathrm{mmol}$ ) according to general procedure 1. Purification of the crude product by flash chromatography (EtOAc/hexane 3:2) afforded 26 (189 mg, 76\% yield) as a white solid. ${ }^{1} \mathrm{H}$ NMR was in agreement with the data reported in the literature. ${ }^{22}$

\section{4-[4'-(Aminosulfonyl)phenyl]-1-(2',3',4',6'-tetra-O-propionyl- $\beta$-D-galactopyranosyl)-1H-1,2,3-}

\section{triazole (27)}

The title compound 27 was prepared from galactopyranosyl azide 7 (178 mg, $0.42 \mathrm{mmol}$ ) and alkyne 11 (81 mg, $0.45 \mathrm{mmol}$ ) according to general procedure 1 in 5 h. Purification of the crude product by flash chromatography (EtOAc/hexane 1:1) afforded 27 (181 mg, 71\% yield) as a white foam. $R_{f}=$ $0.10\left(\right.$ EtOAc/hexane 2:3) $[\alpha]^{25}{ }_{\mathrm{D}}-45\left(c=0.089, \mathrm{CHCl}_{3}\right) .{ }^{1} \mathrm{H}$ NMR $\left(500 \mathrm{MHz}, \mathrm{DMSO}-d_{6}\right): \delta=9.03(\mathrm{~s}$, $1 \mathrm{H}, \mathrm{CH}_{\text {triazole }}$ ), 8.11 (bd, ${ }^{3} \mathrm{~J}=8.6 \mathrm{~Hz}, 2 \mathrm{H}, \mathrm{CH}_{\text {arom }}$ ), 7.91 (bd, ${ }^{3} J=8.6 \mathrm{~Hz}, 2 \mathrm{H}, \mathrm{CH}_{\text {arom }}$ ), 7.38 (s, $2 \mathrm{H}$, $\left.\mathrm{SO}_{2} \mathrm{NH}_{2}\right), 6.37\left(\mathrm{~d},{ }^{3} J_{1^{\prime}, 2^{\prime}}=9.2 \mathrm{~Hz}, 1 \mathrm{H}, \mathrm{H}_{1^{\prime}}\right), 5.68\left(\mathrm{dd} \approx \mathrm{bt},{ }^{3} J_{2^{\prime}, 3^{\prime}}=10.1 \mathrm{~Hz},{ }^{3} J_{2^{\prime}, 1^{\prime}}=9.2 \mathrm{~Hz}, 1 \mathrm{H}, \mathrm{H}_{2^{\prime}}\right), 5.56$ 
(dd, $\left.{ }^{3} J_{3^{\prime}, 2^{\prime}}=10.1 \mathrm{~Hz},{ }^{3} J_{3^{\prime}, 4^{\prime}}=3.5 \mathrm{~Hz}, 1 \mathrm{H}, \mathrm{H}_{3^{\prime}}\right), 5.48\left(\mathrm{bd},{ }^{3} J_{4^{\prime}, 3^{\prime}}=3.4 \mathrm{~Hz}, 1 \mathrm{H}, \mathrm{H}_{4^{\prime}}\right), 4.68\left(\mathrm{dd} \approx \mathrm{bt},{ }^{3} J_{5^{\prime}, 6 a^{\prime}}=\right.$ $\left.7.2 \mathrm{~Hz},{ }^{3} J_{5^{\prime}, 6 b^{\prime}}=5.6 \mathrm{~Hz}, 1 \mathrm{H}, \mathrm{H}_{5^{\prime}}\right), 4.18\left(\mathrm{dd},{ }^{2} J_{6 a^{\prime}, 6 b^{\prime}}=11.5 \mathrm{~Hz},{ }^{3} J_{6 a^{\prime}, 5^{\prime}}=5.6 \mathrm{~Hz}, 1 \mathrm{H}, \mathrm{H}_{6 \mathrm{~b}^{\prime}}\right), 4.07(\mathrm{dd}$, $\left.{ }^{2} J_{6 a^{\prime}, 6 b^{\prime}}=11.5 \mathrm{~Hz},{ }^{3} J_{6 a^{\prime}, 5^{\prime}}=7.2 \mathrm{~Hz}, 1 \mathrm{H}, \mathrm{H}_{6 \mathrm{a}^{\prime}}\right), 2.56-2.51\left(\mathrm{~m}, 2 \mathrm{H}, \mathrm{COCH}_{2} \mathrm{CH}_{3}\right), 2.33-2.26(\mathrm{~m}, 2 \mathrm{H}$, $\left.\mathrm{COCH}_{2} \mathrm{CH}_{3}\right), 2.24-2.51\left(\mathrm{~m}, 2 \mathrm{H}, \mathrm{COCH}_{2} \mathrm{CH}_{3}\right), 2.18-2.02\left(\mathrm{~m}, 2 \mathrm{H}, \mathrm{COCH}_{2} \mathrm{CH}_{3}\right), 1.15$ (t, $J=7.5$ $\mathrm{Hz}, 3 \mathrm{H}, \mathrm{COCH}_{2} \mathrm{CH}_{3}$ ), $1.01-0.95\left(\mathrm{~m}, 6 \mathrm{H}, 2 \times \mathrm{COCH}_{2} \mathrm{CH}_{3}\right), 0.78$ (t, $\left.J=7.5 \mathrm{~Hz}, 3 \mathrm{H}, \mathrm{COCH}_{2} \mathrm{CH}_{3}\right)$. Assignments were confirmed by ${ }^{1} \mathrm{H}-{ }^{1} \mathrm{H}$ gCOSY.${ }^{13} \mathrm{C}$ NMR (500 MHz, DMSO- $d_{6}$ ): $\delta=173.22$, 173.16, 172.4, $172.0\left(4 \times \mathrm{COCH}_{2} \mathrm{CH}_{3}\right), 145.7\left(\mathrm{C}_{1^{\prime \prime} / 4^{\prime \prime}}\right), 143.6\left(\mathrm{C}_{1^{\prime \prime} / 4^{\prime \prime}}\right), 133.1\left(\mathrm{C}_{\text {triazole }}\right), 126.4(2 \times$ $\left.\mathrm{CH}_{\text {arom }}\right)$, $125.6\left(2 \times \mathrm{C}_{\text {arom }}\right), 121.8\left(\mathrm{CH}_{\text {triazole }}\right), 84.5\left(\mathrm{C}_{1^{\prime}}\right), 73.0\left(\mathrm{C}_{5^{\prime}}\right), 70.4\left(\mathrm{C}_{3^{\prime}}\right), 67.8\left(\mathrm{C}_{2^{\prime}}\right), 67.1\left(\mathrm{C}_{4^{\prime}}\right)$, $61.2\left(\mathrm{C}_{6}\right), 26.8,26.7,26.54,26.47\left(4 \times \mathrm{COCH}_{2} \mathrm{CH}_{3}\right), 9.2,8.9,8.8,8.7\left(4 \times \mathrm{COCH}_{2} \mathrm{CH}_{3}\right)$. Assignments were confirmed by ${ }^{1} \mathrm{H}^{-13} \mathrm{C}$ gHSQC. LRMS $\left(\mathrm{ESI}^{+}\right): \mathrm{m} / \mathrm{z}=633[\mathrm{M}+\mathrm{Na}]^{+}$. HRMS (ESI) calcd for $\mathrm{C}_{26} \mathrm{H}_{34} \mathrm{~N}_{4} \mathrm{O}_{11} \mathrm{SNa}$ 633.1837, found 633.1811.

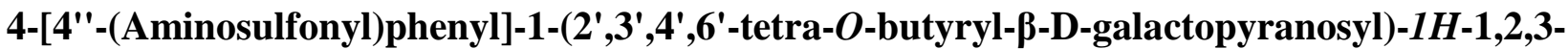

\section{triazole (28)}

The title compound 28 was prepared from galactopyranosyl azide 8 (181 mg, $0.37 \mathrm{mmol}$ ) and alkyne 11 (74 mg, $0.41 \mathrm{mmol}$ ) according to general procedure 1 in $5 \mathrm{~h}$. Purification of the crude product by flash chromatography (EtOAc/hexane 2:3) afforded 28 (181 mg, 71\% yield) as a white solid. $R_{f}=$ 0.10 (EtOAc/hexane 2:3). mp 78-88 ${ }^{\circ} \mathrm{C}(\mathrm{dec}.) .[\alpha]^{25}{ }_{\mathrm{D}}-42\left(c=0.072, \mathrm{CHCl}_{3}\right) .{ }^{1} \mathrm{H}$ NMR $(500 \mathrm{MHz}$, DMSO- $d_{6}$ ): $\delta=9.01$ (s, $1 \mathrm{H}, \mathrm{CH}_{\text {triazole }}$ ), 8.10 (bd, ${ }^{3} J=8.4 \mathrm{~Hz}, 2 \mathrm{H}, \mathrm{CH}_{\text {arom }}$ ), 7.90 (bd, ${ }^{3} J=8.4 \mathrm{~Hz}, 2 \mathrm{H}$, $\mathrm{CH}_{\text {arom }}$ ), $7.38\left(\mathrm{~s}, 2 \mathrm{H}, \mathrm{SO}_{2} \mathrm{NH}_{2}\right), 6.36\left(\mathrm{~d},{ }^{3} J_{1^{\prime}, 2^{\prime}}=9.2 \mathrm{~Hz}, 1 \mathrm{H}, \mathrm{H}_{1^{\prime}}\right), 5.69\left(\mathrm{dd} \approx \mathrm{bt},{ }^{3} J_{2^{\prime}, 3^{\prime}}=10.2 \mathrm{~Hz},{ }^{3} J_{2^{\prime}, 1^{\prime}}=\right.$ $\left.9.2 \mathrm{~Hz}, 1 \mathrm{H}, \mathrm{H}_{2^{\prime}}\right), 5.56\left(\mathrm{dd},{ }^{3} J_{3^{\prime}, 2^{\prime}}=10.2 \mathrm{~Hz},{ }^{3} J_{3^{\prime}, 4^{\prime}}=3.4 \mathrm{~Hz}, 1 \mathrm{H}, \mathrm{H}_{3^{\prime}}\right), 5.48\left(\mathrm{bd},{ }^{3} J_{4^{\prime}, 3^{\prime}}=3.4 \mathrm{~Hz}, 1 \mathrm{H}, \mathrm{H}_{4^{\prime}}\right)$, $4.67\left(\mathrm{dd} \approx \mathrm{bt},{ }^{3} J_{5^{\prime}, 6 a^{\prime}}=7.2 \mathrm{~Hz},{ }^{3} J_{5^{\prime}, 6 b^{\prime}}=5.5 \mathrm{~Hz}, 1 \mathrm{H}, \mathrm{H}_{5^{\prime}}\right), 4.18\left(\mathrm{dd},{ }^{2} J_{6 a^{\prime}, 6 b^{\prime}}=11.5 \mathrm{~Hz},{ }^{3} J_{6 a^{\prime}, 5^{\prime}}=5.5 \mathrm{~Hz}, 1 \mathrm{H}\right.$, $\mathrm{H}_{6 \mathrm{~b}^{\prime}}$ ), $4.07\left(\mathrm{dd},{ }^{2} J_{6 a^{\prime}, 6 \mathrm{~b}^{\prime}}=11.5 \mathrm{~Hz},{ }^{3} J_{6 a^{\prime} 5^{\prime}}=7.2 \mathrm{~Hz}, 1 \mathrm{H}, \mathrm{H}_{6 \mathrm{a}^{\prime}}\right), 2.49-2.45\left(\mathrm{~m}, 2 \mathrm{H}, \mathrm{COCH}_{2} \mathrm{CH}_{2} \mathrm{CH}_{3}\right.$ ), $2.25\left(\mathrm{t}, J=7.4 \mathrm{~Hz}, 2 \mathrm{H}, \mathrm{COCH}_{2} \mathrm{CH}_{2} \mathrm{CH}_{3}\right), 2.22-2.13\left(\mathrm{~m}, 2 \mathrm{H}, \mathrm{COCH}_{2} \mathrm{CH}_{2} \mathrm{CH}_{3}\right), 2.13-1.99(\mathrm{~m}, 2 \mathrm{H}$, 
$\left.\mathrm{COCH}_{2} \mathrm{CH}_{2} \mathrm{CH}_{3}\right), 1.70-1.61\left(\mathrm{~m}, 2 \mathrm{H}, \mathrm{COCH}_{2} \mathrm{CH}_{2} \mathrm{CH}_{3}\right), 1.53-1.43\left(\mathrm{~m}, 4 \mathrm{H}, 2 \times \mathrm{COCH}_{2} \mathrm{CH}_{2} \mathrm{CH}_{3}\right)$, $1.34-1.20\left(\mathrm{~m}, 2 \mathrm{H}, \mathrm{COCH}_{2} \mathrm{CH}_{2} \mathrm{CH}_{3}\right), 0.97\left(\mathrm{t}, J=7.4 \mathrm{~Hz}, 3 \mathrm{H}, \mathrm{COCH}_{2} \mathrm{CH}_{2} \mathrm{CH}_{3}\right), 0.87-0.82(\mathrm{~m}, 6 \mathrm{H}$, $2 \times \mathrm{COCH}_{2} \mathrm{CH}_{2} \mathrm{CH}_{3}$ ), 0.57 (t, $J=7.4 \mathrm{~Hz}, 3 \mathrm{H}, \mathrm{COCH}_{2} \mathrm{CH}_{2} \mathrm{CH}_{3}$ ). Assignments were confirmed by ${ }^{1} \mathrm{H}-$ ${ }^{1} \mathrm{H}$ gCOSY. ${ }^{13} \mathrm{C}$ NMR (500 MHz, DMSO- $\left.d_{6}\right): \delta=172.33,172.29,171.6,171.0(4 \times$ $\left.\mathrm{COCH}_{2} \mathrm{CH}_{2} \mathrm{CH}_{3}\right), 145.7\left(\mathrm{C}_{1^{\prime \prime} / 4^{\prime \prime}}\right), 143.6\left(\mathrm{C}_{1^{\prime \prime} / 4^{\prime \prime}}\right), 133.2\left(\mathrm{C}_{\text {triazole }}\right), 126.4\left(2 \times \mathrm{CH}_{\text {arom }}\right), 125.7(2 \times$ $\left.\mathrm{CH}_{\text {arom }}\right), 121.8\left(\mathrm{CH}_{\text {triazole }}\right), 84.6\left(\mathrm{C}_{1^{\prime}}\right), 73.1\left(\mathrm{C}_{5^{\prime}}\right), 70.3\left(\mathrm{C}_{3^{\prime}}\right), 67.7\left(\mathrm{C}_{2^{\prime}}\right), 67.1\left(\mathrm{C}_{4^{4}}\right), 61.2\left(\mathrm{C}_{6^{\prime}}\right)$, 35.3, 35.2, 35.1, $34.8\left(4 \times \mathrm{COCH}_{2} \mathrm{CH}_{2} \mathrm{CH}_{3}\right), 18.1,17.79,17.78,17.6\left(4 \times \mathrm{COCH}_{2} \mathrm{CH}_{2} \mathrm{CH}_{3}\right), 13.3,13.2$ (2C), $12.8\left(4 \times \mathrm{COCH}_{2} \mathrm{CH}_{2} \mathrm{CH}_{3}\right)$. Assignments were confirmed by ${ }^{1} \mathrm{H}^{-13} \mathrm{C}$ gHSQC. LRMS $\left(\mathrm{ESI}^{+}\right)$: $m / z=689[\mathrm{M}+\mathrm{Na}]^{+}$. HRMS (ESI) calcd for $\mathrm{C}_{30} \mathrm{H}_{42} \mathrm{~N}_{4} \mathrm{O}_{11} \mathrm{SNa}$ 689.2463, found 689.2435.

\section{4-[4"'-(Aminosulfonyl)phenyl]-1-(2',3',4',6'-tetra-O-pentyryl- $\beta$-D-galactopyranosyl)-1H-1,2,3-}

\section{triazole (29)}

The title compound 29 was prepared from galactopyranosyl azide 9 (158 mg, $0.29 \mathrm{mmol}$ ) and alkyne 11 (91mg, $0.50 \mathrm{mmol}$ ) according to general procedure 1 in $5 \mathrm{~h}$. Purification of the crude product by flash chromatography (EtOAc/hexane 3:7) afforded 29 (144 mg, 68\% yield) as a white solid. $R_{f}=$ 0.07 (EtOAc/hexane 3:7). mp 76-79 ${ }^{\circ} \mathrm{C} .[\alpha]^{27} \mathrm{D}-47\left(c=0.089, \mathrm{CHCl}_{3}\right) .{ }^{1} \mathrm{H}$ NMR (600 MHz, DMSO$\left.d_{6}\right): \delta=9.01\left(\mathrm{~s}, 1 \mathrm{H}, \mathrm{CH}_{\text {triazole }}\right), 8.10\left(\mathrm{~d},{ }^{3} J=8.6 \mathrm{~Hz}, 2 \mathrm{H}, \mathrm{CH}_{\text {arom }}\right), 7.91\left(\mathrm{~d},{ }^{3} J=8.6 \mathrm{~Hz}, 2 \mathrm{H}, \mathrm{CH}_{\text {arom }}\right.$ ), $7.39\left(\mathrm{~s}, 2 \mathrm{H}, \mathrm{SO}_{2} \mathrm{NH}_{2}\right), 6.36\left(\mathrm{~d},{ }^{3} J_{1^{\prime}, 2^{\prime}}=9.3 \mathrm{~Hz}, 1 \mathrm{H}, \mathrm{H}_{1^{\prime}}\right), 5.70\left(\mathrm{dd} \approx \mathrm{bt},{ }^{3} J_{2^{\prime}, 3^{\prime}}=10.2 \mathrm{~Hz},{ }^{3} J_{2^{\prime}, 1^{\prime}}=9.3 \mathrm{~Hz}\right.$, $\left.1 \mathrm{H}, \mathrm{H}_{2^{\prime}}\right), 5.56\left(\mathrm{dd},{ }^{3} J_{3^{\prime}, 2^{\prime}}=10.2 \mathrm{~Hz},{ }^{3} J_{3^{\prime}, 4^{\prime}}=3.5 \mathrm{~Hz}, 1 \mathrm{H}, \mathrm{H}_{3^{\prime}}\right), 5.48\left(\mathrm{bd},{ }^{3} J_{4^{\prime}, 3^{\prime}}=3.5 \mathrm{~Hz}, 1 \mathrm{H}, \mathrm{H}_{4^{\prime}}\right), 4.66$ $\left(\mathrm{dd} \approx \mathrm{bt},{ }^{3} J_{5^{\prime}, 6 a^{\prime}}=7.3 \mathrm{~Hz},{ }^{3} J_{5^{\prime}, 6 b^{\prime}}=5.6 \mathrm{~Hz}, 1 \mathrm{H}, \mathrm{H}_{5^{\prime}}\right), 4.13\left(\mathrm{dd},{ }^{2} J_{6 a^{\prime}, 6 b^{\prime}}=11.6 \mathrm{~Hz},{ }^{3} J_{6 a^{\prime}, 5^{\prime}}=5.6 \mathrm{~Hz}, 1 \mathrm{H}\right.$, $\left.\mathrm{H}_{6 \mathrm{~b}^{\prime}}\right), 4.07$ (dd, $\left.{ }^{2} \mathrm{~J}_{6 a^{\prime}, 6 b^{\prime}}=11.6 \mathrm{~Hz},{ }^{3} \mathrm{~J}_{6 a^{\prime}, 5^{\prime}}=7.3 \mathrm{~Hz}, 1 \mathrm{H}, \mathrm{H}_{6 \mathrm{a}^{\prime}}\right), 2.53-2.50(\mathrm{~m}, 2 \mathrm{H}$, $\left.\mathrm{COCH}_{2} \mathrm{CH}_{2} \mathrm{CH}_{2} \mathrm{CH}_{3}\right), 2.29-2.25\left(\mathrm{~m}, 2 \mathrm{H}, \mathrm{COCH}_{2} \mathrm{CH}_{2} \mathrm{CH}_{2} \mathrm{CH}_{3}\right), 2.22-2.17(\mathrm{~m}, 2 \mathrm{H}$, $\left.\mathrm{COCH}_{2} \mathrm{CH}_{2} \mathrm{CH}_{2} \mathrm{CH}_{3}\right), 2.12-2.02\left(\mathrm{~m}, 2 \mathrm{H}, \mathrm{COCH}_{2} \mathrm{CH}_{2} \mathrm{CH}_{2} \mathrm{CH}_{3}\right), 1.65-1.59(\mathrm{~m}, 2 \mathrm{H}$, $\left.\mathrm{COCH}_{2} \mathrm{CH}_{2} \mathrm{CH}_{2} \mathrm{CH}_{3}\right), 1.49-1.41\left(\mathrm{~m}, 4 \mathrm{H}, 2 \times \mathrm{COCH}_{2} \mathrm{CH}_{2} \mathrm{CH}_{2} \mathrm{CH}_{3}\right), 1.41-1.36(\mathrm{~m}, 2 \mathrm{H}$, 
$\left.\mathrm{COCH}_{2} \mathrm{CH}_{2} \mathrm{CH}_{2} \mathrm{CH}_{3}\right), 1.27-1.20\left(\mathrm{~m}, 6 \mathrm{H}, 2 \times \mathrm{COCH}_{2} \mathrm{CH}_{2} \mathrm{CH}_{2} \mathrm{CH}_{3}, \mathrm{COCH}_{2} \mathrm{CH}_{2} \mathrm{CH}_{2} \mathrm{CH}_{3}\right), 0.95-$ 0.90 (m, 5H, $\left.\quad \mathrm{COCH}_{2} \mathrm{CH}_{2} \mathrm{CH}_{2} \mathrm{CH}_{3}, \quad \mathrm{COCH}_{2} \mathrm{CH}_{2} \mathrm{CH}_{2} \mathrm{CH}_{3}\right), \quad 0.83$ (t, $J=7.4 \mathrm{~Hz}, \quad 3 \mathrm{H}$, $\left.\mathrm{COCH}_{2} \mathrm{CH}_{2} \mathrm{CH}_{2} \mathrm{CH}_{3}\right), 0.80\left(\mathrm{t}, J=7.4 \mathrm{~Hz}, 3 \mathrm{H}, \mathrm{COCH}_{2} \mathrm{CH}_{2} \mathrm{CH}_{2} \mathrm{CH}_{3}\right), 0.63(\mathrm{t}, J=7.4 \mathrm{~Hz}, 3 \mathrm{H}$, $\mathrm{COCH}_{2} \mathrm{CH}_{2} \mathrm{CH}_{2} \mathrm{CH}_{3}$ ). Assignments were confirmed by ${ }^{1} \mathrm{H}^{-1} \mathrm{H}$ gCOSY. ${ }^{13} \mathrm{C} \mathrm{NMR}$ (500 MHz, DMSO-d $\left.d_{6}\right): \delta=172.40,172.37,171.7,171.0\left(\mathrm{COCH}_{2} \mathrm{CH}_{2} \mathrm{CH}_{2} \mathrm{CH}_{3}\right), 145.7\left(\mathrm{C}_{1 " / 4}\right), 143.5\left(\mathrm{C}_{1 " / 4}\right)$, $133.2\left(\mathrm{C}_{\text {triazole }}\right), 126.3\left(2 \times \mathrm{CH}_{\text {arom }}\right), 125.6\left(2 \times \mathrm{C}_{\text {arom }}\right), 121.8\left(\mathrm{CH}_{\text {triazole }}\right), 84.5\left(\mathrm{C}_{1^{\prime}}\right), 73.0\left(\mathrm{C}_{5^{\prime}}\right), 70.3$ $\left(\mathrm{C}_{3^{\prime}}\right), 67.7\left(\mathrm{C}_{2^{\prime}}\right), 67.1\left(\mathrm{C}_{4^{\prime}}\right), 61.1\left(\mathrm{C}_{6^{\prime}}\right), 33.04,32.99,32.9,32.7\left(4 \times \mathrm{COCH}_{2} \mathrm{CH}_{2} \mathrm{CH}_{2} \mathrm{CH}_{3}\right)$, 26.6, 26.38, 26.35, $26.2\left(4 \times \mathrm{COCH}_{2} \mathrm{CH}_{2} \mathrm{CH}_{2} \mathrm{CH}_{3}\right), 21.5,21.44,21.41,21.0\left(4 \times \mathrm{COCH}_{2} \mathrm{CH}_{2} \mathrm{CH}_{2} \mathrm{CH}_{3}\right)$, 13.52, 13.45, 13.40, $13.3\left(4 \times \mathrm{COCH}_{2} \mathrm{CH}_{2} \mathrm{CH}_{2} \mathrm{CH}_{3}\right)$. Assignments were confirmed by ${ }^{1} \mathrm{H}_{-}{ }^{13} \mathrm{C}$ gHSQC. LRMS (ESI $\left.{ }^{+}\right): m / z=723[\mathrm{M}+\mathrm{H}]^{+}, 745[\mathrm{M}+\mathrm{Na}]^{+}$. HRMS (ESI) calcd for $\mathrm{C}_{34} \mathrm{H}_{50} \mathrm{~N}_{4} \mathrm{O}_{11} \mathrm{SNa}$ 745.3089, found 745.3089.

\section{4-[4' '-(Aminosulfonyl)phenyl]-1-(2',3',4',6'-tetra-O-(3-methylbutyryl)- $\beta$-D-galactopyranosyl)-}

\section{H-1,2,3-triazole (30)}

The title compound 30 was prepared from galactopyranosyl azide 10 (219 mg, $0.40 \mathrm{mmol}$ ) and alkyne 11 (96 mg, $0.53 \mathrm{mmol}$ ) according to general procedure 1 in $7.5 \mathrm{~h}$. Purification of the crude product by flash chromatography (EtOAc/hexane 3:7) afforded 30 (93 $\mathrm{mg}, 32 \%$ yield) as an offwhite solid. $R_{f}=0.09$ (EtOAc/hexane 3:7). mp 152-154 ${ }^{\circ} \mathrm{C} .[\alpha]^{26}{ }_{\mathrm{D}}-53\left(c=0.072, \mathrm{CHCl}_{3}\right) .{ }^{1} \mathrm{H}$ NMR (500 MHz, DMSO- $\left.d_{6}\right): \delta=9.00$ (s, $1 \mathrm{H}, \mathrm{CH}_{\text {triazole }}$ ), 8.09 (d, $\left.{ }^{3} \mathrm{~J}=8.2 \mathrm{~Hz}, 2 \mathrm{H}, \mathrm{CH}_{\text {arom }}\right), 7.91$ (d, ${ }^{3} J=8.2$ $\mathrm{Hz}, 2 \mathrm{H}, \mathrm{CH}_{\text {arom }}$ ), 7.39 (s, 2H, SO $\left.\mathrm{NH}_{2}\right), 6.37\left(\mathrm{~d},{ }^{3} J_{1^{\prime}, 2^{\prime}}=9.2 \mathrm{~Hz}, 1 \mathrm{H}, \mathrm{H}_{1^{\prime}}\right), 5.72\left(\mathrm{dd} \approx \mathrm{bt},{ }^{3} J_{2^{\prime}, 3^{\prime}}=10.1\right.$ $\left.\mathrm{Hz},{ }^{3} J_{2^{\prime}, 1^{\prime}}=9.3 \mathrm{~Hz}, 1 \mathrm{H}, \mathrm{H}_{2^{\prime}}\right), 5.57\left(\mathrm{dd},{ }^{3} J_{3^{\prime}, 2^{\prime}}=10.1 \mathrm{~Hz},{ }^{3} J_{3^{\prime}, 4^{\prime}}=3.1 \mathrm{~Hz}, 1 \mathrm{H}, \mathrm{H}_{3^{\prime}}\right), 5.49\left(\mathrm{bd},{ }^{3} J_{4^{\prime}, 3^{\prime}}=3.1\right.$ $\left.\mathrm{Hz}, 1 \mathrm{H}, \mathrm{H}_{4^{\prime}}\right), 4.66\left(\mathrm{dd} \approx \mathrm{bt},{ }^{3} J_{5^{\prime}, 6 a^{\prime}}=6.1 \mathrm{~Hz},{ }^{3} J_{5^{\prime}, 6 b^{\prime}}=6.1 \mathrm{~Hz}, 1 \mathrm{H}, \mathrm{H}_{5^{\prime}}\right), 4.11\left(\mathrm{~d},{ }^{3} J_{6 a^{\prime}, 5^{\prime}}=6.1 \mathrm{~Hz}, 2 \mathrm{H}\right.$, $\mathrm{H}_{\left.6 \mathrm{a}^{\prime} / 6 \mathrm{~b}^{\prime}\right),} 2.40$ (m, 2H, $\left.\mathrm{COCH}_{2} \mathrm{CH}\left(\mathrm{CH}_{3}\right)_{2}\right), 2.15$ (d, $\left.J=7.9 \mathrm{~Hz}, \mathrm{COCH}_{2} \mathrm{CH}\left(\mathrm{CH}_{3}\right)_{2}\right), 2.13$ - 2.04 (m, 3H, $\left.\mathrm{COCH}_{2} \mathrm{CH}\left(\mathrm{CH}_{3}\right)_{2}, \quad \mathrm{COCH}_{2} \mathrm{CH}\left(\mathrm{CH}_{3}\right)_{2}\right), \quad 1.98-1.86$ (m, 4H, $2 \times \mathrm{COCH}_{2} \mathrm{CH}\left(\mathrm{CH}_{3}\right)_{2}$, 
$\left.\mathrm{COCH}_{2} \mathrm{CH}\left(\mathrm{CH}_{3}\right)_{2}\right), 1.70-1.59\left(\mathrm{~m}, 1 \mathrm{H}, \mathrm{COCH}_{2} \mathrm{CH}\left(\mathrm{CH}_{3}\right)_{2}\right), 1.02-0.98\left(\mathrm{~m}, 6 \mathrm{H}, \mathrm{COCH}_{2} \mathrm{CH}\left(\mathrm{CH}_{3}\right)_{2}\right)$, $0.88-0.81\left(\mathrm{~m}, 12 \mathrm{H}, 2 \times \mathrm{COCH}_{2} \mathrm{CH}\left(\mathrm{CH}_{3}\right)_{2}\right), 0.63-0.55\left(\mathrm{~m}, 6 \mathrm{H}, \mathrm{COCH}_{2} \mathrm{CH}\left(\mathrm{CH}_{3}\right)_{2}\right)$. Assignments were confirmed by ${ }^{1} \mathrm{H}-{ }^{1} \mathrm{H}$ gCOSY. ${ }^{13} \mathrm{C}$ NMR (500 MHz, DMSO- $\left.d_{6}\right): \delta=171.7,171.6,171.0,170.2$ (4 × $\left.\mathrm{COCH}_{2} \mathrm{CH}\left(\mathrm{CH}_{3}\right)_{2}\right), 145.7\left(\mathrm{C}_{1^{\prime \prime} / 4^{\prime \prime}}\right), 143.5\left(\mathrm{C}_{1^{\prime \prime} / 4^{\prime \prime}}\right), 133.2\left(\mathrm{C}_{\text {triazole }}\right), 126.4\left(2 \times \mathrm{CH}_{\text {arom }}\right), 125.6(2 \times$ $\left.\mathrm{CH}_{\text {arom }}\right), 121.8\left(\mathrm{CH}_{\text {triazole }}\right), 84.6\left(\mathrm{C}_{1^{\prime}}\right), 73.1\left(\mathrm{C}_{5^{\prime}}\right), 70.3\left(\mathrm{C}_{3^{\prime}}\right), 67.6\left(\mathrm{C}_{2^{\prime}}\right), 67.1\left(\mathrm{C}_{4^{\prime}}\right), 61.2\left(\mathrm{C}_{6^{\prime}}\right), 42.32$, 42.29, 42.2, $42.0\left(4 \times \mathrm{COCH}_{2} \mathrm{CH}\left(\mathrm{CH}_{3}\right)_{2}\right), 25.2,25.11,25.07,24.8\left(4 \times \mathrm{COCH}_{2} \mathrm{CH}\left(\mathrm{CH}_{3}\right)_{2}\right), 21.98$ (2C), 21.92 (4C), 21.5, $21.4\left(8 \times \mathrm{COCH}_{2} \mathrm{CH}\left(\mathrm{CH}_{3}\right)_{2}\right)$. Assignments were confirmed by ${ }^{1} \mathrm{H}^{-13} \mathrm{C}$ gHSQC. LRMS $\left(\right.$ ESI $\left.^{+}\right): m / z=723[\mathrm{M}+\mathrm{H}]^{+}$. HRMS (ESI) calcd for $\mathrm{C}_{34} \mathrm{H}_{50} \mathrm{~N}_{4} \mathrm{O}_{11} \mathrm{SNa}$ 745.3089, found 745.3087 .

\section{4-[3"'-(Aminosulfonyl)phenyl]-1-(3-D-galactopyranosyl)-1H-1,2,3-triazole (31)}

The title compound 31 was prepared from triazole $32(58 \mathrm{mg}, 0.11 \mathrm{mmol})$ according to general procedure 2 and isolated as a white solid (38 $\mathrm{mg}, 93 \%) . R_{f}=0.65\left(\mathrm{CH}_{3} \mathrm{CN} / \mathrm{H}_{2} \mathrm{O}\right.$ 9:1). mp 124-126 ${ }^{\circ} \mathrm{C} .[\alpha]^{22}{ }_{\mathrm{D}}+7\left(c=0.013, \mathrm{CH}_{3} \mathrm{OH}\right) .{ }^{1} \mathrm{H}$ NMR $\left(500 \mathrm{MHz}\right.$, DMSO- $\left.d_{6}\right): \delta=8.90\left(\mathrm{~s}, 1 \mathrm{H}, \mathrm{CH}_{\text {triazole }}\right), 8.40$ (bs, $1 \mathrm{H}, \mathrm{H}_{2 "}$ ), 8.10 (bd, ${ }^{3} J_{4^{\prime \prime} / 6^{\prime \prime}, 5^{\prime \prime}}=7.5 \mathrm{~Hz}, 1 \mathrm{H}, \mathrm{H}_{4^{\prime \prime}}$ or 6"), 7.79 (bd, ${ }^{3} J_{4^{\prime \prime} / 6^{\prime \prime}, 5^{\prime \prime}}=7.8 \mathrm{~Hz}, 1 \mathrm{H}, \mathrm{H}_{4^{\prime \prime}}$ or 6"), 7.65 (t, $\left.{ }^{3} J_{5^{\prime \prime}, 4^{\prime \prime} / 6^{\prime \prime}}=7.5 \mathrm{~Hz}, 1 \mathrm{H}, \mathrm{H}_{5^{\prime \prime}}\right), 7.41$ (bs, $2 \mathrm{H}, \mathrm{SO}_{2} \mathrm{NH}_{2}$ ), 5.55 (d, $\left.{ }^{3} J_{1^{\prime}, 2^{\prime}}=9.2 \mathrm{~Hz}, 1 \mathrm{H}, \mathrm{H}_{1^{\prime}}\right), 5.30-5.26$ (m, 1H, OH), 5.08-5.03 (m, 1H, OH), 4.71-4.64 (m, 2H, $2 \times \mathrm{OH}), 4.13-4.07$ (m, 1H, $\left.\mathrm{H}_{2}\right), 3.82-3.78(\mathrm{~m}$, $\left.1 \mathrm{H}, \mathrm{H}_{5^{\prime}}\right), 3.78-3.75\left(\mathrm{~m}, 1 \mathrm{H}, \mathrm{H}_{4^{\prime}}\right), 3.62-3.49\left(\mathrm{~m}, 3 \mathrm{H}, \mathrm{H}_{3^{\prime}}, 6 \mathrm{a}^{\prime}, 6 \mathrm{~b}^{\prime}\right)$. Assignments were confirmed by ${ }^{1} \mathrm{H}-$ ${ }^{1} \mathrm{H}$ gCOSY. ${ }^{13} \mathrm{C}$ NMR (500 MHz, DMSO- $\left.d_{6}\right): \delta=145.2\left(\mathrm{C}_{1 " \text { or 3" }}\right), 144.9\left(\mathrm{C}_{1 " \text { or 3" }}\right), 131.3\left(\mathrm{C}_{\text {triazole }}\right)$, $129.6\left(\mathrm{C}_{5^{\prime \prime}}\right), 128.3\left(\mathrm{C}_{4^{\prime \prime}}\right.$ or 6" $), 124.8\left(\mathrm{C}_{4^{\prime \prime}}\right.$ or 6" $\left.{ }^{\prime \prime}\right), 122.1\left(\mathrm{C}_{2^{\prime \prime}}\right), 121.0\left(\mathrm{CH}_{\text {triazole }}\right), 88.4\left(\mathrm{C}_{1^{\prime}}\right), 78.5\left(\mathrm{C}_{4^{\prime}}\right), 73.6$ $\left(\mathrm{C}_{3^{\prime}}\right), 69.4\left(\mathrm{C}_{2^{\prime}}\right), 68.4\left(\mathrm{C}_{5^{\prime}}\right), 60.4\left(\mathrm{C}_{6^{\prime}}\right)$. Assignments were confirmed by ${ }^{1} \mathrm{H}^{13}{ }^{13} \mathrm{C}$ gHSQC. LRMS $\left(\right.$ ESI $\left.^{+}\right): m / z=409[\mathrm{M}+\mathrm{Na}]^{+}$. HRMS (ESI) calcd for $\mathrm{C}_{14} \mathrm{H}_{18} \mathrm{~N}_{4} \mathrm{O}_{7} \mathrm{SNa}$ 409.0788, found 409.0786. 


\section{4-[3' '-(Aminosulfonyl)phenyl]-1-(2',3',4',6'-tetra-O-acetyl- $\beta$-D-galactopyranosyl)-1H-1,2,3-}

\section{triazole (32)}

The title compound 32 was prepared from galactopyranosyl azide 6 (122 mg, 0.33 mmol) and alkyne 12 (65 mg, $0.36 \mathrm{mmol}$ ) according to general procedure 1 in $4 \mathrm{~h}$. Purification of the crude product by flash chromatography (EtOAc/hexane 3:2) afforded 32 (151 mg, 83\%) as a white solid. $R_{f}=0.21$ (EtOAc/hexane 3:2). mp 120-145 ${ }^{\circ} \mathrm{C}$ (dec.). $[\alpha]^{26}{ }_{\mathrm{D}}-25\left(c=0.1, \mathrm{CHCl}_{3}\right) .{ }^{1} \mathrm{H}$ NMR (500 MHz, DMSO- $d_{6}$ ): $\delta=9.05$ (s, $1 \mathrm{H}, \mathrm{CH}_{\text {triazole }}$ ), 8.42 (bs, $1 \mathrm{H}, \mathrm{H}_{2 "}$ ), 8.13 (bd, ${ }^{3} J_{4^{\prime \prime} / 6^{\prime \prime}, 5^{\prime \prime}}=7.8, \mathrm{~Hz}, 1 \mathrm{H}, \mathrm{H}_{4 "}$ or 6"), 7.81 (bd, ${ }^{3} J_{4^{\prime \prime} / 6^{\prime \prime}, 5^{\prime \prime}}=7.8 \mathrm{~Hz}, 1 \mathrm{H}, \mathrm{H}_{4 "}$ or $\left.6^{\prime \prime}\right), 7.67$ (t, $\left.{ }^{3} J_{5^{\prime \prime}, 4^{\prime \prime} / 6^{\prime \prime}}=7.8 \mathrm{~Hz}, 1 \mathrm{H}, \mathrm{H}_{5 "}\right), 7.43$ (bs, $2 \mathrm{H}, \mathrm{SO}_{2} \mathrm{NH}_{2}$ ), $6.35\left(\mathrm{~d},{ }^{3} J_{1^{\prime}, 2^{\prime}}=9.2 \mathrm{~Hz}, 1 \mathrm{H}, \mathrm{H}_{1^{\prime}}\right), 5.64\left(\mathrm{dd} \approx \mathrm{t},{ }^{3} J_{2^{\prime}, 3^{\prime}}=10.1 \mathrm{~Hz},{ }^{3} J_{2^{\prime}, 3^{\prime}}=9.2 \mathrm{~Hz}, 1 \mathrm{H}, \mathrm{H}_{2^{\prime}}\right), 5.52\left(\mathrm{dd},{ }^{3} J_{3^{\prime}, 2^{\prime}}=\right.$ $\left.10.1 \mathrm{~Hz},{ }^{3} J_{3^{\prime}, 4^{\prime}}=3.2 \mathrm{~Hz}, 1 \mathrm{H}, \mathrm{H}_{3^{\prime}}\right), 5.46\left(\mathrm{bd},{ }^{3} J_{4^{\prime}, 3^{\prime}}=3.2 \mathrm{~Hz}, 1 \mathrm{H}, \mathrm{H}_{4^{\prime}}\right), 4.65\left(\mathrm{dd} \approx \mathrm{bt},{ }^{3} J_{5^{\prime}, 6 a^{\prime}}=7.3 \mathrm{~Hz}\right.$,

$\left.{ }^{3} J_{5^{\prime}, 6 b^{\prime}}=5.0 \mathrm{~Hz}, 1 \mathrm{H}, \mathrm{H}_{5^{\prime}}\right), 4.17\left(\mathrm{dd},{ }^{2} J_{6 a^{\prime}, 6 b^{\prime}}=11.6 \mathrm{~Hz},{ }^{3} J_{6 a^{\prime}, 5^{\prime}}=5.0 \mathrm{~Hz}, 1 \mathrm{H}, \mathrm{H}_{6 \mathrm{~b}^{\prime}}\right), 4.07\left(\mathrm{dd},{ }^{2} J_{6 a^{\prime}, 6 b^{\prime}}=\right.$ $\left.11.6 \mathrm{~Hz},{ }^{3} J_{6 a^{\prime}, 5^{\prime}}=7.3 \mathrm{~Hz}, 1 \mathrm{H}, \mathrm{H}_{6 \mathrm{a}^{\prime}}\right), 2.23,2.00,1.96,1.83\left(4 \times \mathrm{s}, 4 \times 3 \mathrm{H}, 4 \times \mathrm{COCH}_{3}\right)$. Assignments were confirmed by ${ }^{1} \mathrm{H}-{ }^{1} \mathrm{H}$ gCOSY. ${ }^{13} \mathrm{C}$ NMR (500 MHz, DMSO- $\left.d_{6}\right): \delta=170.0,169.88,169.4,168.6$ (4 × $\left.\mathrm{COCH}_{3}\right), 145.7\left(\mathrm{C}_{1 "}\right.$ or 3"), $144.9\left(\mathrm{C}_{1 "}\right.$ or 3"), $130.7\left(\mathrm{C}_{\text {triazole }}\right), 129.7\left(\mathrm{C}_{5}\right), 128.5\left(\mathrm{C}_{4 " \text { or 6" }}\right), 125.2\left(\mathrm{C}_{4 "}\right.$ or 6"), $122.3\left(\mathrm{C}_{2^{\prime \prime}}\right), 121.3\left(\mathrm{CH}_{\text {triazole }}\right), 84.6\left(\mathrm{C}_{1^{\prime}}\right), 73.0\left(\mathrm{C}_{5^{\prime}}\right), 70.0\left(\mathrm{C}_{3^{\prime}}\right), 68.0\left(\mathrm{C}_{2^{\prime}}\right), 67.3\left(\mathrm{C}_{4^{\prime}}\right), 61.5\left(\mathrm{C}_{6^{\prime}}\right)$, 20.44, 20.40, 20.3, $20.0\left(4 \times \mathrm{COCH}_{3}\right)$. Assignments were confirmed by ${ }^{1} \mathrm{H}^{-13} \mathrm{C}$ gHSQC. LRMS $\left(\mathrm{ESI}^{+}\right): \mathrm{m} / \mathrm{z}=555[\mathrm{M}+\mathrm{H}]^{+}, 577[\mathrm{M}+\mathrm{Na}]^{+} . \mathrm{HRMS}(\mathrm{ESI})$ calcd for $\mathrm{C}_{22} \mathrm{H}_{26} \mathrm{~N}_{4} \mathrm{O}_{11} \mathrm{SNa}$ 577.1211, found 577.1221.

\section{4-[3' '-(Aminosulfonyl)phenyl]-1-(2',3',4',6'-tetra-O-propionyl- $\beta$-D-galactopyranosyl)-1H-1,2,3-}

\section{triazole (33)}

The title compound 33 was prepared from galactopyranosyl azide 7 (177 mg, $0.41 \mathrm{mmol}$ ) and alkyne 12 (82 mg, $0.45 \mathrm{mmol}$ ) according to general procedure 1 in $4 \mathrm{~h}$. Purification of the crude product by flash chromatography (EtOAc/hexane 1:1) afforded $33(178 \mathrm{mg}, 71 \%)$ as a white solid. $R_{f}=0.27$ 
(EtOAc/hexane 1:1). mp 78-89 ${ }^{\circ} \mathrm{C}($ dec. $) .[\alpha]^{26}{ }_{\mathrm{D}}-22\left(c=0.067, \mathrm{CHCl}_{3}\right) .{ }^{1} \mathrm{H}$ NMR $(500 \mathrm{MHz}$, DMSO- $d_{6}$ ): $\delta=9.03$ (s, $1 \mathrm{H}, \mathrm{CH}_{\text {triazole }}$ ), 8.41 (bs, $1 \mathrm{H}, \mathrm{H}_{2^{\prime \prime}}$ ), 8.12 (bd, ${ }^{3} J_{4^{\prime \prime} / 6^{\prime \prime}, 5^{\prime \prime}}=7.8, \mathrm{~Hz}, 1 \mathrm{H}, \mathrm{H}_{4^{\prime \prime}}$ or $6^{\prime \prime}$ ),

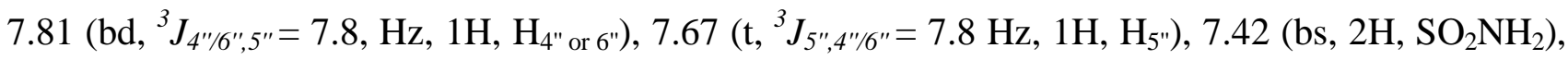
$6.36\left(\mathrm{~d},{ }^{3} J_{1^{\prime}, 2^{\prime}}=9.2 \mathrm{~Hz}, 1 \mathrm{H}, \mathrm{H}_{1^{\prime}}\right), 5.68\left(\mathrm{dd} \approx \mathrm{t},{ }^{3} J_{2^{\prime}, 3^{\prime}}=10.1 \mathrm{~Hz},{ }^{3} J_{2^{\prime}, 3^{\prime}}=9.2 \mathrm{~Hz}, 1 \mathrm{H}, \mathrm{H}_{2^{\prime}}\right), 5.56\left(\mathrm{dd},{ }^{3} J_{3^{\prime}, 2^{\prime}}=\right.$ $\left.10.1 \mathrm{~Hz},{ }^{3} J_{3^{\prime}, 4^{\prime}}=3.3 \mathrm{~Hz}, 1 \mathrm{H}, \mathrm{H}_{3^{\prime}}\right), 5.48\left(\mathrm{bd},{ }^{3} J_{4^{\prime}, 3^{\prime}}=3.3 \mathrm{~Hz}, 1 \mathrm{H}, \mathrm{H}_{4^{\prime}}\right), 4.68\left(\mathrm{dd} \approx \mathrm{bt},{ }^{3} J_{5^{\prime}, 6 a^{\prime}}=7.1 \mathrm{~Hz}\right.$, $\left.{ }^{3} J_{5^{\prime}, 6 b^{\prime}}=5.6 \mathrm{~Hz}, 1 \mathrm{H}, \mathrm{H}_{5^{\prime}}\right), 4.18\left(\mathrm{dd},{ }^{2} J_{6 a^{\prime}, 6 b^{\prime}}=11.5 \mathrm{~Hz},{ }^{3} J_{6 a^{\prime}, 5^{\prime}}=5.6 \mathrm{~Hz}, 1 \mathrm{H}, \mathrm{H}_{6 \mathrm{~b}^{\prime}}\right), 4.07\left(\mathrm{dd},{ }^{2} J_{6 a^{\prime}, 6 b^{\prime}}=\right.$ $\left.11.5 \mathrm{~Hz},{ }^{3} \mathrm{~J}_{6 a^{\prime}, 5^{\prime}}=7.1 \mathrm{~Hz}, 1 \mathrm{H}, \mathrm{H}_{6 \mathrm{a}^{\prime}}\right), 2.56-2.51\left(\mathrm{~m}, 2 \mathrm{H}, \mathrm{COCH}_{2} \mathrm{CH}_{3}\right), 2.32-2.27(\mathrm{~m}, 2 \mathrm{H}$, $\left.\mathrm{COCH}_{2} \mathrm{CH}_{3}\right), 2.26-2.17\left(\mathrm{~m}, 2 \mathrm{H}, \mathrm{COCH}_{2} \mathrm{CH}_{3}\right), 2.17-2.02\left(\mathrm{~m}, 2 \mathrm{H}, \mathrm{COCH}_{2} \mathrm{CH}_{3}\right), 1.15$ (t, $J=7.5$ $\left.\mathrm{Hz}, 3 \mathrm{H}, \mathrm{COCH}_{2} \mathrm{CH}_{3}\right), 1.03-0.94\left(\mathrm{~m}, 6 \mathrm{H}, \mathrm{COCH}_{2} \mathrm{CH}_{3}\right), 0.79\left(\mathrm{t}, \mathrm{J}=7.5 \mathrm{~Hz}, 3 \mathrm{H}, \mathrm{COCH}_{2} \mathrm{CH}_{3}\right)$. Assignments were confirmed by ${ }^{1} \mathrm{H}-{ }^{1} \mathrm{H}$ gCOSY.${ }^{13} \mathrm{C}$ NMR (500 MHz, DMSO- $d_{6}$ ): $\delta=173.23$, 173.16, 172.4, $172.0\left(4 \times \mathrm{COCH}_{2} \mathrm{CH}_{3}\right), 145.7$ ( $\left.\mathrm{C}_{1 " \text { or 3" }}\right), 144.9$ ( $\left.\mathrm{C}_{1 " \text { or 3" }}\right), 130.7$ ( $\left.\mathrm{C}_{\text {triazole }}\right), 129.7\left(\mathrm{C}_{5^{\prime \prime}}\right)$, $128.6\left(\mathrm{C}_{4^{\prime \prime}}\right.$ or 6"), $125.2\left(\mathrm{C}_{4^{\prime \prime}}\right.$ or 6"), $122.2\left(\mathrm{C}_{2^{\prime \prime}}\right), 121.3\left(\mathrm{CH}_{\text {triazole }}\right), 84.5\left(\mathrm{C}_{1^{\prime}}\right), 73.0\left(\mathrm{C}_{5^{\prime}}\right), 70.3\left(\mathrm{C}_{3^{\prime}}\right), 67.9$ $\left(\mathrm{C}_{2^{\prime}}\right), 67.1\left(\mathrm{C}_{4^{\prime}}\right), 61.2\left(\mathrm{C}_{6^{\prime}}\right), 26.8,26.7,26.6,26.5\left(4 \times \mathrm{COCH}_{2} \mathrm{CH}_{3}\right), 9.2,8.9,8.8,8.7(4 \times$ $\left.\mathrm{COCH}_{2} \mathrm{CH}_{3}\right)$. Assignments were confirmed by ${ }^{1} \mathrm{H}^{-13} \mathrm{C}$ gHSQC. LRMS $\left(\mathrm{ESI}^{+}\right): m / z=633[\mathrm{M}+\mathrm{Na}]^{+}$. HRMS (ESI) calcd for $\mathrm{C}_{26} \mathrm{H}_{34} \mathrm{~N}_{4} \mathrm{O}_{11} \mathrm{SNa}$ 633.1837, found 633.1851.

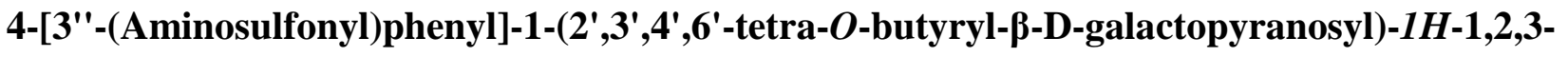 triazole (34)}

The title compound 34 was prepared from galactopyranosyl azide 8 (181 mg, $0.37 \mathrm{mmol})$ and alkyne 12 (76 mg, $0.42 \mathrm{mmol}$ ) according to general procedure $1 \mathrm{in} 4 \mathrm{~h}$. Purification of the crude product by flash chromatography (EtOAc/hexane 2:3) afforded 34 (195 mg, 79\%) as a white solid. $R_{f}=0.22$ (EtOAc/Hexane 2:3). mp 58-70 ${ }^{\circ} \mathrm{C}$ (dec.). $[\alpha]^{26}$ - $35\left(c=0.072, \mathrm{CHCl}_{3}\right) .{ }^{1} \mathrm{H}$ NMR (500 MHz, DMSO- $d_{6}$ ): $\delta=9.02$ (s, $1 \mathrm{H}, \mathrm{CH}_{\text {triazole }}$ ), 8.40 (bs, $1 \mathrm{H}, \mathrm{H}_{2^{\prime \prime}}$ ), 8.11 (bd, ${ }^{3} \mathrm{~J}^{\prime \prime} / 6^{\prime \prime}, 5^{\prime \prime}=7.8, \mathrm{~Hz}, 1 \mathrm{H}, \mathrm{H}_{4^{\prime \prime}}$ or 6"),

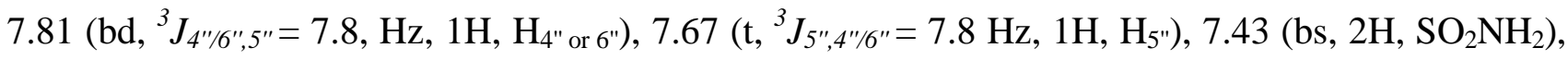


$6.36\left(\mathrm{~d},{ }^{3} J_{1^{\prime}, 2^{\prime}}=9.2 \mathrm{~Hz}, 1 \mathrm{H}, \mathrm{H}_{1^{\prime}}\right), 5.69\left(\mathrm{dd} \approx \mathrm{t},{ }^{3} J_{2^{\prime}, 3^{\prime}}=10.1 \mathrm{~Hz},{ }^{3} J_{2^{\prime}, 3^{\prime}}=9.2 \mathrm{~Hz}, 1 \mathrm{H}, \mathrm{H}_{2^{\prime}}\right), 5.57\left(\mathrm{dd},{ }^{3} J_{3^{\prime}, 2^{\prime}}=\right.$ $\left.10.1 \mathrm{~Hz},{ }^{3} J_{3^{\prime}, 4^{\prime}}=3.2 \mathrm{~Hz}, 1 \mathrm{H}, \mathrm{H}_{3^{\prime}}\right), 5.49\left(\mathrm{bd},{ }^{3} J_{4^{\prime}, 3^{\prime}}=3.2 \mathrm{~Hz}, 1 \mathrm{H}, \mathrm{H}_{4^{\prime}}\right), 4.67\left(\mathrm{dd} \approx \mathrm{bt},{ }^{3} J_{5^{\prime}, 6 a^{\prime}}=7.2 \mathrm{~Hz}\right.$, $\left.{ }^{3} J_{5^{\prime}, 6 b^{\prime}}=5.5 \mathrm{~Hz}, 1 \mathrm{H}, \mathrm{H}_{5^{\prime}}\right), 4.14\left(\mathrm{dd},{ }^{2} J_{6 a^{\prime}, 6 b^{\prime}}=11.4 \mathrm{~Hz},{ }^{3} J_{6 a^{\prime}, 5^{\prime}}=5.5 \mathrm{~Hz}, 1 \mathrm{H}, \mathrm{H}_{6 b^{\prime}}\right), 4.09$ (dd, ${ }^{2} J_{6 a^{\prime}, 6 b^{\prime}}=$ $\left.11.4 \mathrm{~Hz},{ }^{3} \mathrm{~J}_{6 a^{\prime}, 5^{\prime}}=7.2 \mathrm{~Hz}, 1 \mathrm{H}, \mathrm{H}_{6 \mathrm{a}^{\mathrm{a}}}\right), 2.50-2.45\left(\mathrm{~m}, 2 \mathrm{H}, \mathrm{COCH}_{2} \mathrm{CH}_{2} \mathrm{CH}_{3}\right), 2.29-2.22(\mathrm{~m}, 2 \mathrm{H}$, $\left.\mathrm{COCH}_{2} \mathrm{CH}_{2} \mathrm{CH}_{3}\right) 2.20-2.16\left(\mathrm{~m}, 2 \mathrm{H}, \mathrm{COCH}_{2} \mathrm{CH}_{2} \mathrm{CH}_{3}\right), 2.11-2.01$ (m, 2H, $\left.\mathrm{COCH}_{2} \mathrm{CH}_{2} \mathrm{CH}_{3}\right), 1.70-$ $1.61\left(\mathrm{~m}, 2 \mathrm{H}, \mathrm{COCH}_{2} \mathrm{CH}_{2} \mathrm{CH}_{3}\right), 1.54-1.44\left(\mathrm{~m}, 4 \mathrm{H}, \mathrm{COCH}_{2} \mathrm{CH}_{2} \mathrm{CH}_{3}\right), 1.35-1.21(\mathrm{~m}, 2 \mathrm{H}$, $\mathrm{COCH}_{2} \mathrm{CH}_{2} \mathrm{CH}_{3}$ ), $0.87-0.80$ (m, 9H, $\left.\mathrm{COCH}_{2} \mathrm{CH}_{2} \mathrm{CH}_{3}\right), 0.59$ (t, $J=7.4 \mathrm{~Hz}, 3 \mathrm{H}, \mathrm{COCH}_{2} \mathrm{CH}_{2} \mathrm{CH}_{3}$ ). Assignments were confirmed by ${ }^{1} \mathrm{H}-{ }^{1} \mathrm{H}$ gCOSY. ${ }^{13} \mathrm{C}$ NMR (500 MHz, DMSO- $d_{6}$ ): $\delta=172.3,171.6$, 171.0, $170.3\left(4 \times \mathrm{COCH}_{2} \mathrm{CH}_{2} \mathrm{CH}_{3}\right), 145.7\left(\mathrm{C}_{1 "}\right.$ or 3"), 144.9 ( $\mathrm{C}_{1 "}$ or 3"), $130.7\left(\mathrm{C}_{\text {triazole }}\right), 129.7\left(\mathrm{C}_{5^{\prime \prime}}\right)$, $128.6\left(\mathrm{C}_{4^{\prime \prime}}\right.$ or 6"), $125.2\left(\mathrm{C}_{4^{\prime \prime}}\right.$ or 6" $), 122.2\left(\mathrm{C}_{2^{\prime \prime}}\right), 121.3\left(\mathrm{CH}_{\text {triazole }}\right), 84.6\left(\mathrm{C}_{1^{\prime}}\right), 73.0\left(\mathrm{C}_{5^{\prime}}\right), 70.3\left(\mathrm{C}_{3^{\prime}}\right), 67.7$ $\left(\mathrm{C}_{2^{\prime}}\right), 67.1\left(\mathrm{C}_{4^{\prime}}\right), 61.1\left(\mathrm{C}_{6^{\prime}}\right), 35.2,35.1,35.0,34.8\left(4 \times \mathrm{COCH}_{2} \mathrm{CH}_{2} \mathrm{CH}_{3}\right), 18.1,17.8(2 \mathrm{C}), 17.6(4 \times$ $\left.\mathrm{COCH}_{2} \mathrm{CH}_{2} \mathrm{CH}_{3}\right), 13.3,13.2$ (2C), $12.8\left(4 \times \mathrm{COCH}_{2} \mathrm{CH}_{2} \mathrm{CH}_{3}\right)$. Assignments were confirmed by ${ }^{1} \mathrm{H}-$ ${ }^{13} \mathrm{C}$ gHSQC. LRMS $\left(\mathrm{ESI}^{+}\right): m / z=667[\mathrm{M}+\mathrm{Na}]^{+}, 689[\mathrm{M}+\mathrm{Na}]^{+}$. HRMS (ESI) calcd for $\mathrm{C}_{30} \mathrm{H}_{42} \mathrm{~N}_{4} \mathrm{O}_{11} \mathrm{SNa}$ 689.2463, found 689.2494.

\section{4-[3"'-(Aminosulfonyl)phenyl]-1-(2',3',4',6'-tetra-O-pentyryl- $\beta$-D-galactopyranosyl)-1H-1,2,3- triazole (35)}

The title compound 35 was prepared from galactopyranosyl azide 9 (148 mg, $0.27 \mathrm{mmol}$ ) and alkyne 12 (85 mg, $0.47 \mathrm{mmol}$ ) according to general procedure $1 \mathrm{in} 4 \mathrm{~h}$. Purification of the crude product by flash chromatography (EtOAc/hexane 3:7) afforded $35(104 \mathrm{mg}, 53 \%)$ as a transparent gum. $R_{f}=$ 0.15 (EtOAc/hexane 3:7). mp $41-51{ }^{\circ} \mathrm{C}(\mathrm{dec}.) .[\alpha]^{27}{ }_{\mathrm{D}}-26\left(c=0.089, \mathrm{CHCl}_{3}\right) .{ }^{1} \mathrm{H}$ NMR $(500 \mathrm{MHz}$, DMSO- $d_{6}$ ): $\delta=9.01$ (s, $1 \mathrm{H}, \mathrm{CH}_{\text {triazole }}$ ), 8.40 (bs, $1 \mathrm{H}, \mathrm{H}_{2^{\prime \prime}}$ ), 8.11 (bd, ${ }^{3} \mathrm{~J}^{\prime \prime} / 6^{\prime \prime}, 5^{\prime \prime}=7.8, \mathrm{~Hz}, 1 \mathrm{H}, \mathrm{H}_{4^{\prime \prime}}$ or 6"),

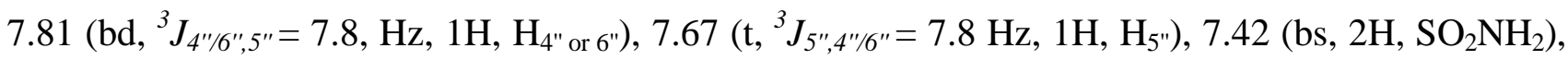
$6.36\left(\mathrm{~d},{ }^{3} J_{1^{\prime}, 2^{\prime}}=9.2 \mathrm{~Hz}, 1 \mathrm{H}, \mathrm{H}_{1^{\prime}}\right), 5.70\left(\mathrm{dd} \approx \mathrm{t},{ }^{3} J_{2^{\prime}, 3^{\prime}}=10.2 \mathrm{~Hz},{ }^{3} J_{2^{\prime}, 3^{\prime}}=9.2 \mathrm{~Hz}, 1 \mathrm{H}, \mathrm{H}_{2^{\prime}}\right), 5.56\left(\mathrm{dd},{ }^{3} J_{3^{\prime}, 2^{\prime}}=\right.$ 
$\left.10.2 \mathrm{~Hz},{ }^{3} J_{3^{\prime}, 4^{\prime}}=3.2 \mathrm{~Hz}, 1 \mathrm{H}, \mathrm{H}_{3^{\prime}}\right), 5.48\left(\mathrm{bd},{ }^{3} J_{4^{\prime}, 3^{\prime}}=3.1 \mathrm{~Hz}, 1 \mathrm{H}, \mathrm{H}_{4^{\prime}}\right), 4.66\left(\mathrm{dd} \approx \mathrm{bt},{ }^{3} J_{5^{\prime}, 6 a^{\prime}}=6.3 \mathrm{~Hz}\right.$, $\left.{ }^{3} J_{5^{\prime}, 6 b^{\prime}}=6.3 \mathrm{~Hz}, 1 \mathrm{H}, \mathrm{H}_{5^{\prime}}\right), 4.16-4.05\left(\mathrm{~m}, 2 \mathrm{H}, \mathrm{H}_{6 \mathrm{a}^{\prime}}, \mathrm{H}_{6 b^{\prime}}\right), 2.53-2.50\left(\mathrm{~m}, 2 \mathrm{H}, \mathrm{COCH}_{2} \mathrm{CH}_{2} \mathrm{CH}_{2} \mathrm{CH}_{3}\right)$, 2.27 (t, $J=7.2 \mathrm{~Hz}, 2 \mathrm{H}, \mathrm{COCH}_{2} \mathrm{CH}_{2} \mathrm{CH}_{2} \mathrm{CH}_{3}$ ), $2.23-2.16$ (m, $2 \mathrm{H}, \mathrm{COCH}_{2} \mathrm{CH}_{2} \mathrm{CH}_{2} \mathrm{CH}_{3}$ ), $2.13-2.02$ (m, $2 \mathrm{H}, \mathrm{COCH}_{2} \mathrm{CH}_{2} \mathrm{CH}_{2} \mathrm{CH}_{3}$ ), $1.66-1.57$ (m, 2H, $\left.\mathrm{COCH}_{2} \mathrm{CH}_{2} \mathrm{CH}_{2} \mathrm{CH}_{3}\right), 1.50-1.42$ (m, 4H, $2 \times$ $\left.\mathrm{COCH}_{2} \mathrm{CH}_{2} \mathrm{CH}_{2} \mathrm{CH}_{3}\right), 1.42-1.34\left(\mathrm{~m}, 2 \mathrm{H}, \mathrm{COCH}_{2} \mathrm{CH}_{2} \mathrm{CH}_{2} \mathrm{CH}_{3}\right), 1.29-1.17(\mathrm{~m}, 6 \mathrm{H}, 2 \times$ $\mathrm{COCH}_{2} \mathrm{CH}_{2} \mathrm{CH}_{2} \mathrm{CH}_{3}, \mathrm{COCH}_{2} \mathrm{CH}_{2} \mathrm{CH}_{2} \mathrm{CH}_{3}$ ), $1.00-0.93$ (m, 2H, $\mathrm{COCH}_{2} \mathrm{CH}_{2} \mathrm{CH}_{2} \mathrm{CH}_{3}$ ), 0.92 (t, $J=$ $\left.7.3 \mathrm{~Hz}, 3 \mathrm{H}, \mathrm{COCH}_{2} \mathrm{CH}_{2} \mathrm{CH}_{2} \mathrm{CH}_{3}\right), 0.86-0.76\left(\mathrm{~m}, 6 \mathrm{H}, 2 \times \mathrm{COCH}_{2} \mathrm{CH}_{2} \mathrm{CH}_{2} \mathrm{CH}_{3}\right), 0.64$ (t, $J=7.3 \mathrm{~Hz}$, $3 \mathrm{H}, \mathrm{COCH}_{2} \mathrm{CH}_{2} \mathrm{CH}_{2} \mathrm{CH}_{3}$ ). Assignments were confirmed by ${ }^{1} \mathrm{H}-{ }^{1} \mathrm{H}$ gCOSY. ${ }^{13} \mathrm{C}$ NMR (500 MHz, DMSO-d $\left.)_{6}\right): \delta=172.42,172.40,171.7,171.1\left(4 \times \mathrm{COCH}_{2} \mathrm{CH}_{2} \mathrm{CH}_{2} \mathrm{CH}_{3}\right), 145.7\left(\mathrm{C}_{1 "}\right.$ or 3"), $144.9\left(\mathrm{C}_{1 " \text { or }}\right.$ 3"), 130.7 ( $\left.\mathrm{C}_{\text {triazole }}\right), 129.7\left(\mathrm{C}_{5^{\prime \prime}}\right), 128.6\left(\mathrm{C}_{4^{\prime \prime}}\right.$ or 6"), 125.2 ( $\mathrm{C}_{4^{\prime \prime}}$ or 6"), $122.2\left(\mathrm{C}_{2 "}\right), 121.3\left(\mathrm{CH}_{\text {triazole }}\right), 84.5$ $\left(\mathrm{C}_{1^{\prime}}\right), 73.0\left(\mathrm{C}_{5^{\prime}}\right), 70.3\left(\mathrm{C}_{3^{\prime}}\right), 67.7\left(\mathrm{C}_{2^{\prime}}\right), 67.1\left(\mathrm{C}_{4^{\prime}}\right), 61.1\left(\mathrm{C}_{6^{\prime}}\right), 33.02,32.99,32.90,32.7(4 \times$ $\left.\mathrm{COCH}_{2} \mathrm{CH}_{2} \mathrm{CH}_{2} \mathrm{CH}_{3}\right)$, 26.6, 26.38, 26.36, $26.2\left(4 \times \mathrm{COCH}_{2} \mathrm{CH}_{2} \mathrm{CH}_{2} \mathrm{CH}_{3}\right)$, 21.5, 21.44, 21.41, 21.1 (4 $\left.\times \mathrm{COCH}_{2} \mathrm{CH}_{2} \mathrm{CH}_{2} \mathrm{CH}_{3}\right), 13.54,13.46,13.41,13.3\left(4 \times \mathrm{COCH}_{2} \mathrm{CH}_{2} \mathrm{CH}_{2} \mathrm{CH}_{3}\right)$. Assignments were confirmed by ${ }^{1} \mathrm{H}^{-13} \mathrm{C}$ gHSQC. LRMS $\left(\mathrm{ESI}^{+}\right): \mathrm{m} / \mathrm{z}=723[\mathrm{M}+\mathrm{H}]^{+}$. HRMS (ESI) calcd for $\mathrm{C}_{34} \mathrm{H}_{50} \mathrm{~N}_{4} \mathrm{O}_{11} \mathrm{SNa}$ 745.3089, found 745.3104.

\section{4-[3"'-(Aminosulfonyl)phenyl]-1-(2',3',4',6'-tetra-O-(3-methylbutyryl)- $\beta$-D-galactopyranosyl)-}

\section{H-1,2,3-triazole (36)}

The title compound 36 was prepared from galactopyranosyl azide 10 (255 mg, $0.47 \mathrm{mmol})$ and alkyne 12 (96 mg, $0.53 \mathrm{mmol}$ ) according to general procedure $1 \mathrm{in} 4 \mathrm{~h}$. Purification of the crude product by flash chromatography (EtOAc/hexane 3:7) afforded $36278 \mathrm{mg}, 82 \%$ ) as an off-white solid. $R_{f}=0.30$ (EtOAc/hexane 2:3). mp 76-80 ${ }^{\circ} \mathrm{C} \cdot[\alpha]^{27} \mathrm{D}-34\left(c=0.089, \mathrm{CHCl}_{3}\right) \cdot{ }^{1} \mathrm{H}$ NMR (500 MHz, DMSO- $\left.d_{6}\right): \delta=9.01$ (s, $\left.1 \mathrm{H}, \mathrm{CH}_{\text {triazole }}\right), 8.39$ (bs, $\left.1 \mathrm{H}, \mathrm{H}_{2^{\prime \prime}}\right), 8.10$ (bd, ${ }^{3} J_{4^{\prime \prime} / 6^{\prime \prime}, 5^{\prime}}=7.8, \mathrm{~Hz}, 1 \mathrm{H}, \mathrm{H}_{4^{\prime \prime}}$ 
or 6"), 7.81 (bd, ${ }^{3} J_{4^{\prime \prime} / 6^{\prime \prime}, 5^{\prime}}=7.8, \mathrm{~Hz}, 1 \mathrm{H}, \mathrm{H}_{4^{\prime \prime}}$ or 6"), $7.67\left(\mathrm{t},{ }^{3} \mathrm{~J}_{5^{\prime \prime}, 4^{\prime \prime} / 6^{\prime}}=7.8 \mathrm{~Hz}, 1 \mathrm{H}, \mathrm{H}_{5^{\prime \prime}}\right), 7.43$ (bs, $2 \mathrm{H}$, $\left.\mathrm{SO}_{2} \mathrm{NH}_{2}\right), 6.36\left(\mathrm{~d},{ }^{3} J_{1^{\prime}, 2^{\prime}}=9.2 \mathrm{~Hz}, 1 \mathrm{H}, \mathrm{H}_{1^{\prime}}\right), 5.72\left(\mathrm{dd} \approx \mathrm{t},{ }^{3} J_{2^{\prime}, 3^{\prime}}=10.2 \mathrm{~Hz},{ }^{3} J_{2^{\prime}, 1^{\prime}}=9.2 \mathrm{~Hz}, 1 \mathrm{H}, \mathrm{H}_{2^{\prime}}\right), 5.57$ (dd, $\left.{ }^{3} J_{3^{\prime}, 2^{\prime}}=10.2 \mathrm{~Hz},{ }^{3} J_{3^{\prime}, 4^{\prime}}=3.1 \mathrm{~Hz}, 1 \mathrm{H}, \mathrm{H}_{3^{\prime}}\right), 5.49\left(\mathrm{bd},{ }^{3} J_{4^{\prime}, 3^{\prime}}=3.1 \mathrm{~Hz}, 1 \mathrm{H}, \mathrm{H}_{4^{\prime}}\right), 4.66\left(\mathrm{dd} \approx \mathrm{bt},{ }^{3} J_{5^{\prime}, 6 a^{\prime}}=\right.$ $\left.6.3 \mathrm{~Hz},{ }^{3} J_{5^{\prime}, 6 b^{\prime}}=6.3 \mathrm{~Hz}, 1 \mathrm{H}, \mathrm{H}_{5^{\prime}}\right), 4.11\left(\mathrm{~d},{ }^{3} J_{6 \mathrm{a}^{\prime} / 6 b^{\prime}, 5^{\prime}}=6.3 \mathrm{~Hz}, 2 \mathrm{H}, \mathrm{H}_{6 \mathrm{a}^{\prime} / 6 b^{\prime}}\right), 2.42-2.38(\mathrm{~m}, 2 \mathrm{H}$, $\left.\mathrm{COCH}_{2} \mathrm{CH}\left(\mathrm{CH}_{3}\right)_{2}\right), 2.18-2.06\left(\mathrm{~m}, 5 \mathrm{H}, 2 \times \mathrm{COCH}_{2} \mathrm{CH}\left(\mathrm{CH}_{3}\right)_{2}, \mathrm{COCH}_{2} \mathrm{CH}\left(\mathrm{CH}_{3}\right)_{2}\right), 1.98-1.87(\mathrm{~m}$, $\left.4 \mathrm{H}, 2 \times \mathrm{COCH}_{2} \mathrm{CH}\left(\mathrm{CH}_{3}\right)_{2}, \mathrm{COCH}_{2} \mathrm{CH}\left(\mathrm{CH}_{3}\right)_{2}\right), 1.70-162\left(\mathrm{~m}, 1 \mathrm{H}, \mathrm{COCH}_{2} \mathrm{CH}\left(\mathrm{CH}_{3}\right)_{2}\right), 1.00$ (dd, $J=$ $\left.6.6 \mathrm{~Hz}, J=2.7 \mathrm{~Hz}, 6 \mathrm{H}, \mathrm{COCH}_{2} \mathrm{CH}\left(\mathrm{CH}_{3}\right)_{2}\right), 0.87-0.82\left(\mathrm{~d}, J=6.6 \mathrm{~Hz}, 12 \mathrm{H}, 2 \times \mathrm{COCH}_{2} \mathrm{CH}\left(\mathrm{CH}_{3}\right)_{2}\right)$, $0.60\left(\right.$ d, $\left.J=6.6 \mathrm{~Hz}, J=2.7 \mathrm{~Hz}, 6 \mathrm{H}, \mathrm{COCH}_{2} \mathrm{CH}\left(\mathrm{CH}_{3}\right)_{2}\right)$. Assignments were confirmed by ${ }^{1} \mathrm{H}-{ }^{1} \mathrm{H}$ gCOSY. ${ }^{13} \mathrm{C}$ NMR (500 MHz, DMSO- $\left.d_{6}\right): \delta=171.7,171.6,171.0,170.2\left(4 \times \mathrm{COCH}_{2} \mathrm{CH}\left(\mathrm{CH}_{3}\right)_{2}\right)$,

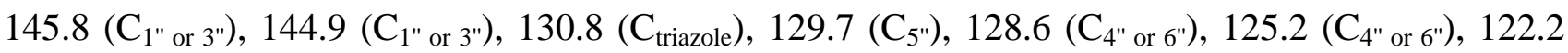
( $\left.\mathrm{C}_{2^{\prime \prime}}\right), 121.3\left(\mathrm{CH}_{\text {triazole }}\right), 84.6\left(\mathrm{C}_{1^{\prime}}\right), 73.1\left(\mathrm{C}_{5^{\prime}}\right), 70.3\left(\mathrm{C}_{3^{\prime}}\right), 67.6\left(\mathrm{C}_{2^{\prime}}\right), 67.1\left(\mathrm{C}_{4^{\prime}}\right), 61.2\left(\mathrm{C}_{6^{\prime}}\right), 42.30,42.28$, 42.2, $42.0\left(\mathrm{COCH}_{2} \mathrm{CH}\left(\mathrm{CH}_{3}\right)_{2}\right), 25.2,25.11,25.08,24.8\left(4 \times \mathrm{COCH}_{2} \mathrm{CH}\left(\mathrm{CH}_{3}\right)_{2}\right), 22.4$ (2C), 21.9 (4C), 21.53, $21.47\left(8 \times \mathrm{COCH}_{2} \mathrm{CH}\left(\mathrm{CH}_{3}\right)_{2}\right)$. Assignments were confirmed by ${ }^{1} \mathrm{H}^{-1}{ }^{13} \mathrm{C}$ gHSQC. LRMS $\left(\mathrm{ESI}^{+}\right): \mathrm{m} / \mathrm{z}=745[\mathrm{M}+\mathrm{Na}]^{+}$. HRMS (ESI) calcd for $\mathrm{C}_{34} \mathrm{H}_{50} \mathrm{~N}_{4} \mathrm{O}_{11} \mathrm{SNa}$ 745.3089, found 745.3079 .

CA Inhibition Assay. An Applied Photophysics stopped-flow instrument was used for assaying the CA-catalyzed $\mathrm{CO}_{2}$ hydration activity as previously reported. ${ }^{5 \mathrm{c}, 20} \mathrm{IC}_{50}$ values were obtained from dose response curves working at seven different concentrations of test compound, by fitting the curves using PRISM (www.graphpad.com) and non-linear least squares methods, values represent the mean of at least three different determinations. The inhibition constants $\left(K_{\mathrm{i}}\right)$ were then derived by using the Cheng-Prusoff equation, ${ }^{23}$ as follows: $K_{\mathrm{i}}=\mathrm{IC}_{50} /\left(1+[\mathrm{s}] / K_{\mathrm{m}}\right)$ where $[\mathrm{S}]$ represents the $\mathrm{CO}_{2}$ concentration at which the measurement was carried out, and $K_{\mathrm{m}}$ the concentration of substrate at which the enzyme activity is at half maximal. 
In Vitro Metabolic Stability. Human liver microsomes (Xenotech, Tokyo, Japan) were suspended in $0.1 \mathrm{M}$ phosphate buffer ( $\mathrm{pH} 7.4$ ) at a final protein concentration of $0.4 \mathrm{mg} / \mathrm{mL}$ and incubated with compounds $(1 \mu \mathrm{M})$ at $37{ }^{\circ} \mathrm{C}$. An NADPH-regenerating system $(1 \mathrm{mg} / \mathrm{mL}$ NADP, $1 \mathrm{mg} / \mathrm{mL}$ glucose6-phosphate, $1 \mathrm{U} / \mathrm{mL}$ glucose-6-phosphate dehydrogenase) and $\mathrm{MgCl}_{2}(0.67 \mathrm{mg} / \mathrm{mL})$ was added to initiate the metabolic reactions, which were subsequently quenched with ice-cold acetonitrile at time points ranging from 2 - 60 min. Samples were also incubated in the absence of co-factor to monitor for non-cytochrome P450-mediated metabolism in the microsomal matrix. Samples were then centrifuged and the concentration of parent compound remaining in the supernatant monitored by LCMS. The first order rate constant for substrate depletion was determined by fitting the data to an exponential decay function and these values were used to calculate the in vitro intrinsic clearance (CLint).

In Vitro Plasma Stability. Human plasma was separated from whole blood procured from the Australian Red Cross Blood Service and was stored frozen at $-80{ }^{\circ} \mathrm{C}$. On the day of the experiment, frozen plasma was thawed in a water bath maintained at $37{ }^{\circ} \mathrm{C}$ and plasma from three different donors was pooled prior to confirming plasma esterase activity by monitoring the conversion of $p$ nitrophenyl acetate to p-nitrophenol. Each test compound was spiked into a separate aliquot of human plasma to a nominal concentration of $5000 \mathrm{ng} / \mathrm{mL}$ (final DMSO and acetonitrile concentrations were 0.2 and $0.4 \%$ (v/v), respectively). Spiked plasma was incubated at $37{ }^{\circ} \mathrm{C}$ for $4 \mathrm{~h}$, and at various time points, duplicate plasma samples were taken and immediately snap-frozen in dry ice. All plasma samples were stored frozen $\left(-20^{\circ} \mathrm{C}\right)$ until analysis by UPLC-MS (using a Waters/Micromass Xevo triple quadrupole mass spectrometer) relative to calibration standards prepared in blank human plasma. At each sample time, the average concentration of test compound 
(based on duplicate samples) was expressed as a \% compound remaining, and these data were used to calculate the apparent degradation half-life by fitting to an exponential decay function.

Chromatographic Human Serum Albumin Binding. Protein binding characteristics were estimated by correlation of their chromatographic retention properties on an immobilised human serum albumin (HSA) column against the characteristics of a series of standard compounds with known protein binding properties. The method employed is a gradient HPLC method based on the approach developed by Valko. ${ }^{21}$ Chromatographic retention was assessed using a ChromTech Chiral HSA column $(3.0 \times 50 \mathrm{~mm}, 5 \mu \mathrm{m})$ with mobile phase comprised of a mixture of aqueous ammonium acetate (pH 7.4) and iso-propanol. The assay uses 13 standard compounds of known plasma protein binding (PPB) values ranging from 5.4 to $99.5 \%$ bound.

Permeability Measurements. Caco-2 cells (passage 31) were seeded onto $0.3 \mathrm{~cm}^{2}$ polycarbonate filter transwells at a density of 60,000 cells/well. Confluent cell monolayers were obtained 21 days post-seeding. The integrity of the cell monolayers was determined by measuring the transepithelial electrical resistance (TEER) and only monolayers with TEER values of $>300 \Omega . \mathrm{cm}^{2}$ were utilized. The permeability of nadolol and propranolol (low and high permeability markers, respectively) was also assessed using a subset of wells from the same batch as those used to assess the test compounds. Permeability experiments were performed using Hanks balanced salt solution containing $20 \mathrm{mM}$ HEPES (pH 7.4) in both the apical and basolateral chambers and permeability was assessed in the apical to basolateral direction in the absence and presence of $4 \% \mathrm{BSA}$ in the basolateral chamber using an initial donor solution concentration of $20 \mu \mathrm{M}$ for $13,15 \mu \mathrm{M}$ for 14 , and $8 \mu \mathrm{M}$ for 15 . Test compound solubility in the transport buffer was confirmed prior to the experiment. Compound flux was determined over 90 min with samples taken from the acceptor chamber at 5, 15, 30, 45, 60 and 
90 min. At each sample time, the volume of acceptor solution removed was replaced with blank transport buffer and acceptor concentrations were corrected for this dilution. Donor samples were taken at the start and completion of the experiment. The amount of compound transported was quantitated by LC-MS.

Supporting Information. ${ }^{1} \mathrm{H}$ and ${ }^{13} \mathrm{C}$ NMR spectra of new compounds. This material is available free of charge via the Internet at http://pubs.acs.org.

\section{Acknowledgements}

This research was financed by the Australian Research Council (Grant number DP110100071 to SAP and SAC, FT10100185 to S-AP), Griffith University (Student Scholarship to CJC) and an EU grant of the 7th framework program (Metoxia project to CTS).

\section{Corresponding authors}

S.-A.P. Telephone: +61 73735 7825; e-mail: s.poulsen@griffith.edu.au.

C.T.S. Telephone: +39 055457 3005; e-mail: claudiu.supuran@unifi.it

\section{Abbreviations}

CuAAC, copper-catalyzed azide alkyne cycloaddition; CA, carbonic anhydrase; $K_{\mathrm{i}}$, inhibition constants; HLMs, human liver microsomes; HSA, human serum albumin; CYP450, cytochrome P450; cHSA, chromatographic human serum albumin binding; $\mathrm{CL}_{\text {int }}$, in vitro intrinsic clearance; TEER, transepithelial electrical resistance 


\section{References}

1. Alterio, V.; Di Fiore, A.; D’Ambrosio, K.; Supuran, C. T.; De Simone, G., Multiple binding modes of inhibitors to carbonic anhydrases: How to design specific drugs targeting 15 different isoforms? Chem. Rev. 2012, 112, 4421-4468.

2. (a) Wykoff, C. C.; Beasley, N. J.; Watson, P. H.; Turner, K. J.; Pastorek, J.; Sibtain, A.; Wilson, G. D.; Turley, H.; Talks, K. L.; Maxwell, P. H.; Pugh, C. W.; Ratcliffe, P. J.; Harris, A. L., Hypoxiainducible expression of tumor-associated carbonic anhydrases. Cancer Res. 2000, 60, 7075-7083; (b) Potter, C. P. S.; Harris, A. L., Diagnostic, prognostic and therapeutic implications of carbonic anhydrases in cancer. Br. J. Cancer 2003, 89, 2-7.

3. Neri, D.; Supuran, C. T., Interfering with $\mathrm{pH}$ regulation in tumours as a therapeutic strategy. Nat. Rev. Drug Discov. 2011, 10, 767-777.

4. Apte, S.; Chein, F. T.; Graves, E. E., Molecular imaging of hypoxia: Strategies for probe design and application. Curr. Org. Synth. 2011, 8, 593-603.

5. (a) Scozzafava, A.; Menabuoni, L.; Mincione, F.; Briganti, F.; Mincione, G.; Supuran, C. T., Carbonic anhydrase inhibitors. Synthesis of water-soluble, topically effective, intraocular pressurelowering aromatic/heterocyclic sulfonamides containing cationic or anionic moieties: Is the tail more important than the ring? J. Med. Chem. 1999, 42, 2641-2650; (b) Winum, J.-Y.; Poulsen, S.-A.; Supuran, C. T., Therapeutic applications of glycosidic carbonic anhydrase inhibitors. Med. Res. Rev. 2009, 29, 419-435; (c) Lopez, M.; Bornaghi, L. F.; Innocenti, A.; Vullo, D.; Charman, S. A.; Supuran, C. T.; Poulsen, S.-A., Sulfonamide linked neoglycoconjugates - A new class of inhibitors for cancer-associated carbonic anhydrases. J. Med. Chem. 2010, 53, 2913-2926; (d) Wilkinson, B. L.; Bornaghi, L. F.; Houston, T. A.; Innocenti, A.; Supuran, C. T.; Poulsen, S.-A., A novel class of carbonic anhydrase inhibitors: Glycoconjugate benzene sulfonamides prepared by "click-tailing". $J$. 
Med. Chem. 2006, 49, 6539-6548; (e) Singer, M.; Lopez, M.; Bornaghi, L. F.; Innocenti, A.; Vullo, D.; Supuran, C. T.; Poulsen, S.-A., Inhibition of carbonic anhydrase isozymes with benzene sulfonamides incorporating thio, sulfinyl and sulfonyl glycoside moieties. Bioorg. Med. Chem. Lett. 2009, 19, 2273-2276; (f) Salmon, A. J.; Williams, M. L.; Maresca, A.; Supuran, C. T.; Poulsen, S.A., Synthesis of glycoconjugate carbonic anhydrase inhibitors by ruthenium-catalyzed azide-alkyne 1,3-dipolar cycloaddition. Bioorg. Med. Chem. Lett. 2011, 21, 6058-6061; (g) Wilkinson, B. L.; Bornaghi, L. F.; Houston, T. A.; Innocenti, A.; Vullo, D.; Supuran, C. T.; Poulsen, S.-A., Carbonic anhydrase inhibitors: inhibition of isozymes I, II, and IX with triazole-linked O-glycosides of benzene sulfonamides. J. Med. Chem. 2007, 50, 1651-1657; (h) Wilkinson, B. L.; Innocenti, A.; Vullo, D.; Supuran, C. T.; Poulsen, S.-A., Inhibition of carbonic anhydrases with glycosyltriazole benzene sulfonamides. J. Med. Chem. 2008, 51, 1945-1953.

6. Kerns, E. H.; Di, L., Drug-like Properties: Concepts, Structure Design and Methods: from ADME to Toxicity Optimization. Academic Press: London, 2008.

7. (a) Böhmig, G. A.; Krieger, P.-M.; Säemann, M. D.; Ullrich, R.; Karimi, H.; Wekerle, T.; Mühlbacher, F.; Zlabinger, G. J., Stable prodrugs of $n$-butyric acid: suppression of $\mathrm{T}$ cell alloresponses in vitro and prolongation of heart allograft sumval in a fully allogeneic rat strain combination. Transplant Immunology 1999, 7, 221-227; (b) Sampathkumar, S.-G.; Campbell, C. T.; Weier, C.; Yarema, K. J., Short-chain fatty acid-hexosamine cancer prodrugs: the sugar matters! Drugs of the Future 2006, 31, 1-18; (c) Pouillart, P.; Cerutti, I.; Ronco, G.; Villa, P.; Chany, C., Butyric monosaccharide ester-induced cell differentiation and anti-tumor activity in mice. Importance of their prolonged biological effect for clinical applications in cancer therapy. Int. J. Cancer 1991, 49, 89-95.

8. (a) Bencharit, S.; Edwards, C. C.; Morton, C. L.; Howard-Williams, E. L.; Kuhn, P.; Potter, P. M.; Redinbo, M. R., Multisite Promiscuity in the Processing of endogenous substrates by human 
carboxylesterase 1. J. Mol. Biol. 2006, 363, 201-214; (b) Rillahan, C. D.; Antonopoulos, A.; Lefort, C. T.; Sonon, R.; Azadi, P.; Ley, K.; Dell, A.; Haslam, S., M; Paulson, J. C., Global metabolic inhibitors of sialyl- and fucosyltransferases remodel the glycome. Nat. Chem. Biol. 2012, 8, 661-668. 9. Kolb, H. C.; Finn, M.; Sharpless, K. B., Click chemistry: Diverse chemical function from a few good reactions. Angew. Chem. Int. Ed. 2001, 40, 2004-2021.

10. Meldal, M.; Tornoe, C. W., Cu-Catalyzed azide-alkyne cycloaddition. Chem. Rev. 2008, 108, 2952-3015.

11. (a) Bräse, S.; Gil, C.; Knepper, K.; Zimmermann, V., Organic Azides: An exploding diversity of a unique class of compounds. Angew. Chem., Int. Ed. Engl. 2005, 44, 5188-5240; (b) AragaoLeoneti, V.; Campo, V. L.; Adriane S. Gomes, A. S.; Field, R. A.; Carvalho, I., Application of copper(I)-catalysed azide/alkyne cycloaddition (CuAAC) 'click chemistry’ in carbohydrate drug and neoglycopolymer synthesis. Tetrahedron 2010, 66, 9475-9492; (c) Prescher, J. A.; Dube, D. H.; Bertozzi, C. R., Chemical remodelling of cell surfaces in living animals. Nature 2004, 430, 873-877; (d) Beckmann, H. S. G.; Wittmann, V., Azides in Carbohydrate Chemistry. In Organic Azides Syntheses and Applications, Bräse, S.; Banert, K., Eds. John Wiley \& Sons, Ltd.: Chichester, 2010; pp 469-491.

12. Paulsen, H.; Györgydeák, Z.; Friedmann, M., Konformationsanalyse, III. exo-anomerer effekt und circulardichroismus von glycopyranosylaziden. Chem. Ber. 1974, 107, 1568-1578.

13. Carroux, C. J.; Moeker, J.; Motte, J.; Lopez, M.; Bornaghi, L. F.; Katneni, K.; Ryan, E.; Morizzi, J.; Shackleford, D. M.; Charman, S. A.; Poulsen, S.-A., Synthesis of acylated glycoconjugates as templates to investigate in vitro biopharmaceutical properties. Bioorg. Med. Chem. Lett. 2013, 23, 455-459.

14. (a) Wilkinson, B. L.; Bornaghi, L. F.; Poulsen, S. A.; Houston, T. A., Synthetic utility of glycosyl triazoles in carbohydrate chemistry. Tetrahedron 2006, 62, 8115-8125; (b) Wilkinson, B. L.; 
Bornaghi, L. F.; Houston, T. A.; Innocenti, A.; Vullo, D.; Supuran, C. T.; Poulsen, S.-A., Inhibition of membrane-associated carbonic anhydrase isozymes IX, XII and XIV with a library of glycoconjugate benzenesulfonamides. Bioorg. Med. Chem. Lett. 2007, 17, 987-992.

15. (a) Lopez, M.; Salmon, A.; Supuran, C.; Poulsen, S., Carbonic Anhydrase Inhibitors Developed Through 'Click Tailing'. Curr. Pharm. Des. 2010, 16, 3277-3287; (b) Morris, J. C.; Chiche, J.; Grellier, C.; Lopez, M.; Bornaghi, L. F.; Maresca, A.; Supuran, C. T.; Pouyssegur, J.; Poulsen, S.-A., Targeting Hypoxic Tumor Cell Viability with Carbohydrate-Based Carbonic Anhydrase IX and XII Inhibitors. J. Med. Chem. 2011, 54, 6905-6918; (c) Poulsen, S.-A.; Wilkinson, B. L.; Innocenti, A.; Vullo, D.; Supuran, C. T., Inhibition of human mitochondrial carbonic anhydrases VA and VB with para-(4-phenyltriazole-1-yl)-benzenesulfonamide derivatives. Bioorg. Med. Chem. Lett. 2008, 18, 4624-4627; (d) Salmon, A. J.; Wiliams, M. L.; Wu, Q. K.; Morizzi, J.; Gregg, D.; Charman, S. A.; Vullo, D.; Supuran, C. T.; Poulsen, S.-A., Metallocene-based inhibitors of cancer-associated carbonic anhydrase enzymes IX and XII. J. Med. Chem. 2012, 55, 5506-5517.

16. (a) Supuran, C. T., Carbonic anhydrases: novel therapeutic applications for inhibitors and activators. Nat. Rev. Drug Discov. 2008, 7, 168-181; (b) Krishnamurthy, V. M.; Kaufman, G. K.; Urbach, A. R.; Gitlin, I.; Gudiksen, K. L.; Weibel, D. B.; Whitesides, G. M., Carbonic anhydrase as a model for biophysical and physical-organic studies of proteins and protein-Ligand binding. Chem. Rev. 2008, 108, 946-1051.

17. Tron, G.; Pirali, T.; Billington, R.; Canonico, P.; Sorba, G.; Genazzani, A., Click chemistry reactions in medicinal chemistry: Applications of the 1,3-dipolar cycloaddition between azides and alkynes. Med. Res. Rev. 2008, 28, 278-308.

18. Wischeler, J. S.; Sun, D.; Sandner, N. U.; Linne, U.; Heine, A.; Koert, U.; Klebe, G., Stereo- and Regioselective azide/alkyne cycloadditions in carbonic anhydrase II via tethering, monitored by crystallography and mass spectrometry. Chem. -Eur. J. 2011, 17, 5842-5851. 
19. Zemplén, G., Degradation of the reducing bioses. I. Direct determination of the constitution of cellobiose. Ber. Deutsch. Chem. Ges. 1926, 59, 1254-1266.

20. Khalifah, R. G., The carbon dioxide hydration activity of carbonic anhydrase. J. Biol. Chem. 1971, 246, 2561-2573.

21. Valko, K.; Nunhuck, S.; Bevan, C.; Abraham, M. H.; Reynolds, D. P., Fast gradient HPLC method to determine compounds binding to human serum albumin. Relationships with octanol/water and immobilized artificial membrane lipophilicity. J. Pharm. Sci. 2003, 92, 2236-2248.

22. Poulsen, S. -A.; Wilkinson, B. L.; Innocenti, A.; Vullo, D.; Supuran, C. T. Inhibition of human mitochondrial carbonic anhydrases VA and VB with para-(4-phenyltriazole-1-yl)benzenesulfonamide derivatives. Bioorg. Med. Chem. Lett. 2008, 18, 4624-4627.

23. Cheng, Y.; Prusoff, W. H., Relationship between the inhibition constant $\left(\mathrm{K}_{\mathrm{i}}\right)$ and the concentration of inhibitor which causes 50 per cent inhibition $\left(\mathrm{IC}_{50}\right)$ of an enzymatic reaction. Biochem. Pharmacol. 1973, 22, 3099. 